$\begin{array}{ll} & \text { ABSTRACT } \\ \text { Title of Dissertation: } & \text { PERIPHERAL NEURAL CODING } \\ & \text { STRATEGIES FOR SPECTRAL ANALYSIS } \\ & \text { AND SOUND SOURCE LOCATION IN THE } \\ & \text { NON-TELEOST BONY FISH, ACIPENSER } \\ & \text { FULVESCENS } \\ & \text { Michaela Meyer, Doctor of Philosophy, } 2008 \\ & \\ & \text { Professor Arthur N. Popper, Department of } \\ \text { Birected By: } & \text { Professor University of Maryland, CP and } \\ & \text { Institute, Loyola University of Chicago }\end{array}$

This work is an investigation of coding strategies for spectral analysis and sound source location in Acipenser fulvescens, the lake sturgeon. A. fulvescens belongs to one of the few extant non-teleost ray-finned fishes. The sturgeon taxonomic family, the (Acipenseridae), has a phylogenetic history that dates back about 200 million years. Studies on sensory coding in any species of this family or any other non-teleost species have not been conducted before. Thus, this is the first study on peripheral coding strategies by the auditory system in a non-teleost bony fish.

For this study, a shaker system, similar to that used in previous experiments on teleosts, was used to simulate particle motion of sound at the ears and auditory periphery of A. fulvescens. Simultaneously, electrophysiological recordings of isolated single units from the eighth nerve were obtained. Peripheral coding strategies for spectral analysis and sound source location in A. fulvescens resembled those found in teleosts. Frequency data resembled the characteristics found for auditory afferents (with preferences for lower frequencies) in land vertebrates. In addition, the auditory 
periphery in A. fulvescens appears to be well suited to encode the intensity of sound. In terms of mechanisms for sound source location, eighth nerve afferents responded to directional stimuli in a cosine-like manner (as in teleosts), which can generally serve as the basis for coding the location of a sound source. Certain differences to teleosts were also found in A. fulvescens and these may have implications for the mechanisms for sound source location in azimuth.

The common physiological characteristics between A. fulvescens, teleosts, and land vertebrates may reflect important functions (that are part of the process of auditory scene analysis) of the auditory system that have been conserved throughout the evolution of vertebrates. 


\title{
PERIPHERAL NEURAL CODING STRATEGIES FOR SPECTRAL ANALYSIS AND SOUND SOURCE LOCATION IN THE NON-TELEOST BONY FISH, ACIPENSER FULVESCENS.
}

\author{
By \\ Michaela Meyer \\ Dissertation submitted to the Faculty of the Graduate School of the \\ University of Maryland, College Park, in partial fulfillment \\ of the requirements for the degree of \\ Doctor of Philosophy \\ 2008
}

\author{
Professor Arthur N. Popper, Chair \\ Professor Richard R. Fay, Co-Chair \\ Professor Catherine E. Carr \\ Professor Avis H. Cohen \\ Professor William Hodos \\ Professor David D. Yager
}

Advisory Committee: 
(C) Copyright by Michaela Meyer 2008 


\section{Dedication}

I dedicate this dissertation to my husband Kaushik Ghose and my family for being very supportive of my work and life goals. I also dedicate this work in special memory of my late paternal grandmother Erika Meyer and my maternal grand aunt Hedi Westphal. They were fantastic role models for me. Both were strong women of the highest integrity who contributed to the recovery of a country destroyed by two World Wars. 


\section{Acknowledgements}

My deepest thanks go to my two advisors, Dr. Arthur N. Popper and Dr.

Richard R. Fay. Dr. Popper suggested that I apply for graduate studies at an American graduate school, and offered me a position as a Ph.D. student in his laboratory at the University of Maryland. Without his offer I might not have started such an exciting journey in the United States (within an excellent scientific community fulfilling a dream of mine to do research in the U.S.). I thank Dr. Fay for the generous gesture in lending me his valuable and sensitive shaker system for experiments, thereby offering me an opportunity to do fulfilling science (addressing topics that I had dreamed about since I heard of Dr. Popper's and Dr. Fay's work). I thank both of my mentors for their friendship, support, their excellent guidance in the writing process of my thesis, many inspiring discussions about the work, and their trust in me throughout my time in graduate school. They have worked together as a team for 36 years and I was very fortunate to receive the combined wisdom from both of them.

Many special thanks to Dr. Catherine Carr for her continuous support (including critical evaluation of data, offering equipment, and just being there for me whenever I had a question) and her great enthusiasm about my research.

Special thanks to my other committee-members: Dr. Avis Cohen for giving life-advice and for her warm, supportive attitude towards students; Dr. William Hodos for special advice in critical situations, his superb teaching, and also for his warm and supportive nature (and his jokes); and Dr. David Yager for a great interest in my work, for stimulating discussions, and for expressing his understanding in how 
challenging it is to balance good-quality teaching with difficult electrophysiological experiments. I never felt lost having the support of these mentors and committee members.

Besides my committee-members, I want to thank Dr. Cynthia Moss for being a role model to me especially with regard to supporting students. Very many thanks to Dr. Joelle Presson, Dr. Kate McLeod, and all my lab members for stimulating discussions and feedback to my work. Outside the community of the University of Maryland, I am very grateful for many scientific discussions with Dr. Peggy EddsWalton and Dr. Catherine McCormick

My deepest thanks go to my husband Kaushik Ghose for his continuous support throughout graduate school, and especially for standing by my side during the last two years. It is an extreme gift (from God) to find a person like him who is a fantastic colleague, superb friend, and loving, supportive husband - all at the same time. I thank him for the many lessons in Matlab (and night-supply of food during experiments). In addition, he and my grand aunt Hedi Westphal added significantly to my financial support for the last two years.

I feel very blessed to have found many friends in and outside of my lab in graduate school. I thank these friends and my family for providing me with invaluable support and balance - reminding me of the many different and colorful aspects that life is all about.

Finally, I also thank the Parmly Hearing Institute (of Loyola University Chicago) for financial support (in form of a Parmly Hearing Scholar fellowship) during a three months stay. Special thanks go to the Wisconsin Department of Natural 
Resources and especially Steven Fajfer, for kindly provided me with lake sturgeon for several years. Many thanks go to Dr. Darlene Ketten for the CT scans. 


\section{Table of Contents}

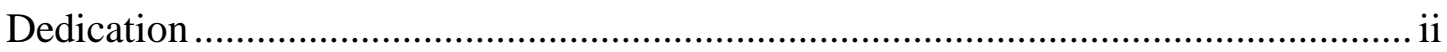

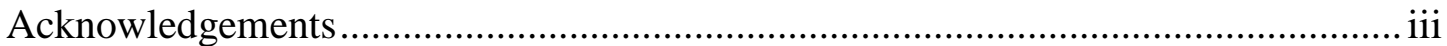

Table of Contents ................................................................................................

Chapter I: Introduction to the Dissertation ............................................................ 1

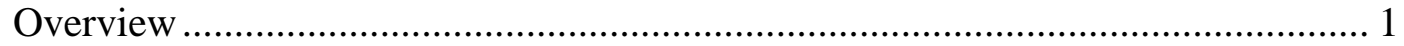

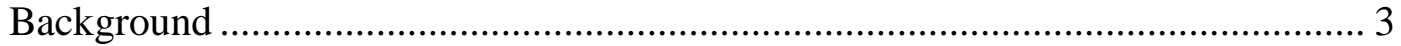

Significance of spectral analysis and sound source location................................ 3

Peripheral auditory structures in teleost and non-teleost bony fishes .................. 6

Sound detection mechanisms in fishes............................................................. 8

Frequency tuning and directional selectivity of eighth nerve fibers in fish ...... 11

Systematic position and special features of sturgeons ..................................... 14

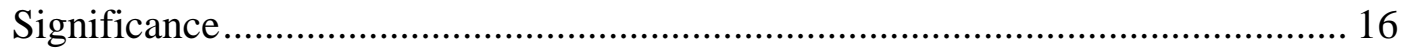

Introductory method section ............................................................................ 17

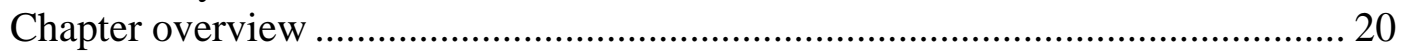

Chapter II: Frequency tuning and intensity coding of sound in the auditory periphery

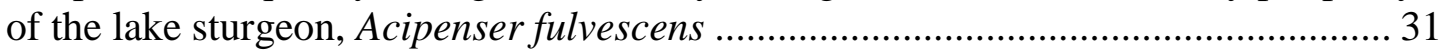

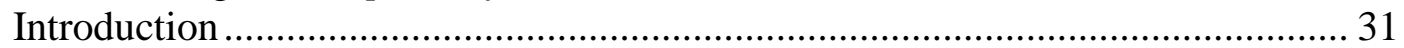

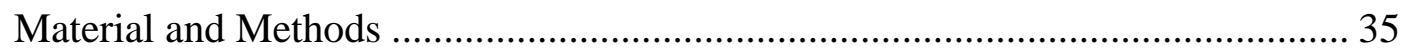

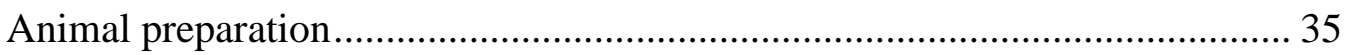

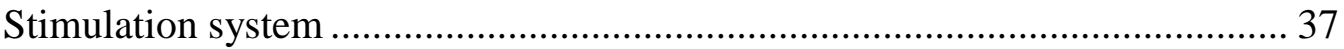

Extracellular recording and data acquisition .................................................... 39

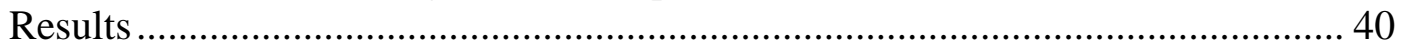

Frequency selectivity of responses................................................................. 41

Displacement, velocity, and acceleration tuning curves .................................... 46

Response level functions .............................................................................. 46

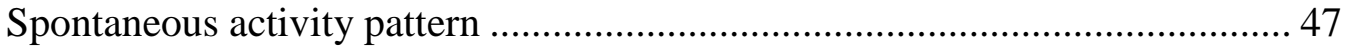

Temporal response pattern, interval distribution, and phase locking in

responding afferents ……............................................................................ 48

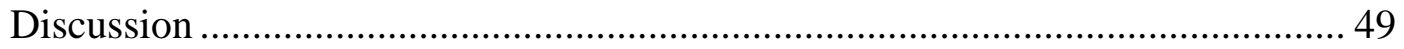

Spike rate versus $Z$ based observations .......................................................... 49

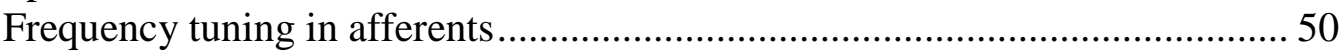

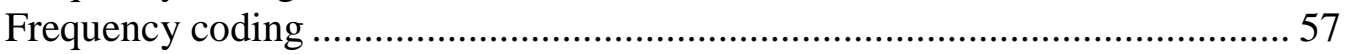

Displacement, velocity, or acceleration detectors? .............................................5 59

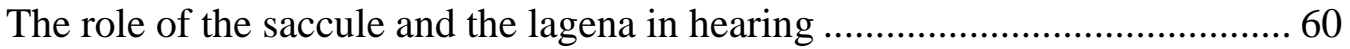

Response level functions and coding of level ..................................................... 61

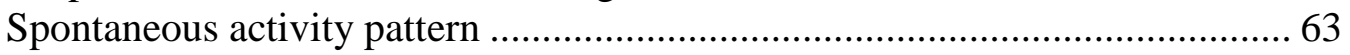

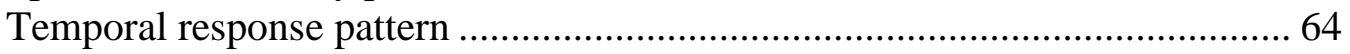

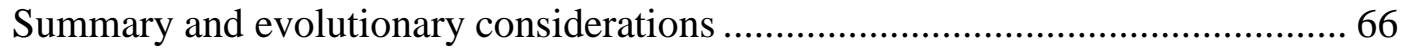

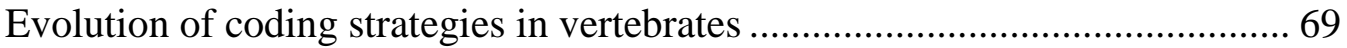

Chapter III: Coding of sound direction in the auditory periphery of the lake sturgeon,

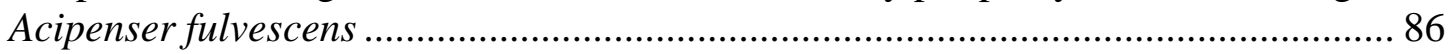

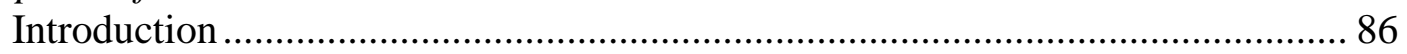

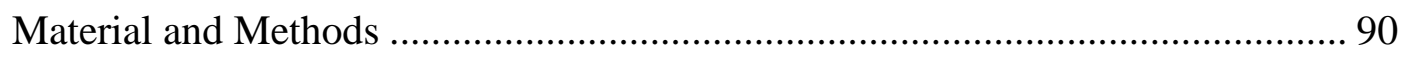




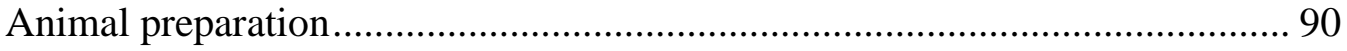

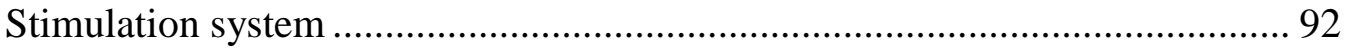

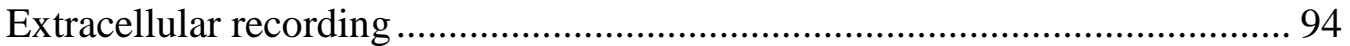

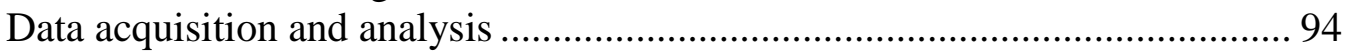

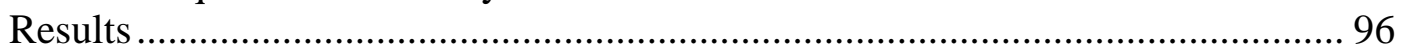

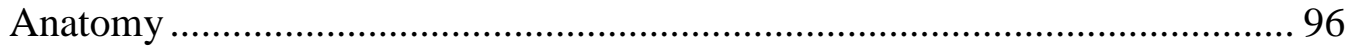

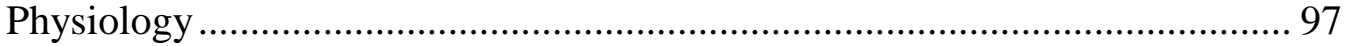

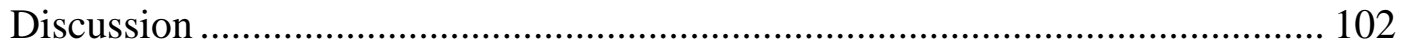

Spontaneous activity and sensitivity ................................................ 102

Directional and non-directional afferents.............................................. 104

Coding for sound source location.......................................................... 106

Dependence of response strength $\mathrm{Z}$ and phase angle on level and spontaneous

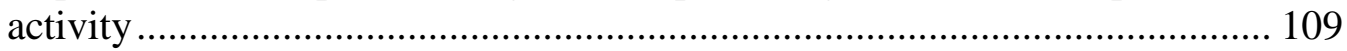

Summary and evolutionary consequences ................................................... 111

Chapter IV: A mathematical model to quantify directional and non-directional response profiles in Acipenser fulvescens, the lake sturgeon ................................ 127

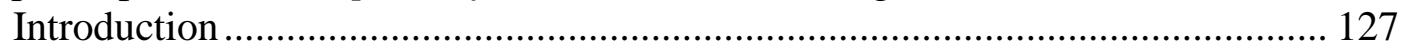

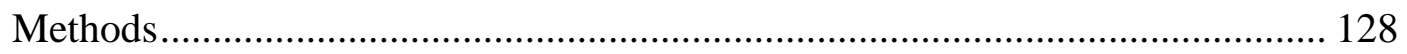

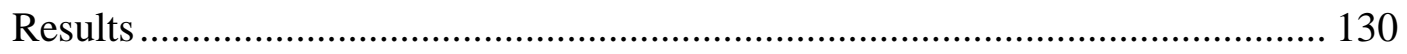

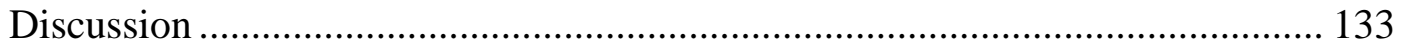

Chapter V: Summary, Final Discussion, and Conclusion .................................... 144

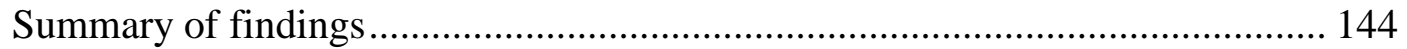

Research implications: functional and evolutionary insights......................... 150

Functional implications .............................................................. 150

Evolutionary implications ............................................................... 154

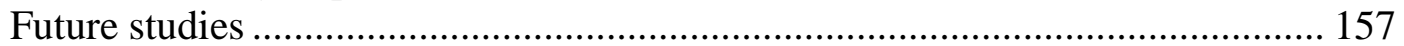

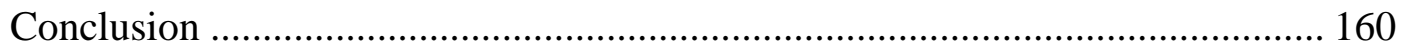

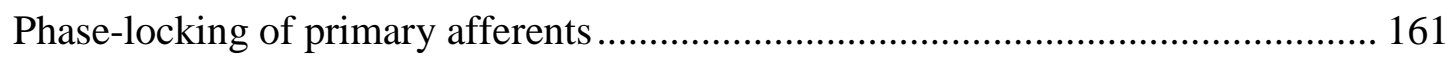

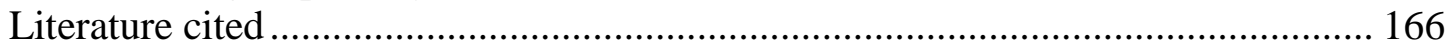




\section{List of Tables}

Chapter 2

Table 2.1 Overview of correlations between parameters....................78

Chapter 4

Table 4.1 Distribution of units according to certain categories that are based on

how K-values changed with level for DRPs.....................143 


\section{List of Figures}

Chapter 1

Figure 1.1

Figure 1.2

Figure 1.3

Figure 1.4

Figure 1.5

Figure 1.6

Figure 1.7

Chapter 2

Figure 2.1

Figure 2.2

Figure 2.3

Figure 2.4

Figure 2.5

Figure 2.6

Figure 2.7

Figure 2.8

Figure 2.9

Chapter 3

Figure 3.1

Figure 3.2

Figure 3.3

Figure 3.4

Figure 3.5

Figure 3.6

Figure 3.7

Figure 3.8

Figure 3.9

Figure 3.10

Chapter 4

Figure 4.1

Figure 4.2

Figure 4.3

Figure 4.4
Phylogenetic relationship of extant vertebrate lineages.............22

Ear of Acipenser sturio, drawing by Retzius $1881 \ldots \ldots \ldots \ldots \ldots \ldots . . . . .23$

Saccular maculae of teleost and non-teleost fish species............24

Photograph and schematic representation of the shaker system......25

Right ear of Acipenser fulvescens...............................27

Action potential traces of a single unit............................28

Vertical and horizontal plane directional response profiles.........29

Example of an isolevel response function using spike rate and Z.....72

Frequency responses of four units..............................73

Isolevel frequency response function of unit $\mathrm{tt} 7 \ldots \ldots \ldots \ldots \ldots \ldots \ldots . . . \ldots 75$

Distributions of mono-peak functions............................76

Sharpness of tuning curves decreasing with CF....................77

Tuning curves of three different units plotted as $\mathrm{dB}$ re displacement, velocity and acceleration........................................79

Response level functions.......................................81

Examples of Interspike interval histograms of background activity...82

Examples of response pattern.

$.83-85$

Right ear and innervation of Acipenser sturio, innervation and hair cell orientation of the saccule and lagena in A. fulvescens...............115

Distribution of spontaneous rates...............................117

Distribution of absolute displacement thresholds..................118

Correlation between spontaneous rate and threshold................119

Directional response profiles in the horizontal and vertical planes...121

Two afferents representing extreme cases in DRP shape...........122

Examples of non-directional units with Z-values lower than five....123

Distributions of Bas in the vertical and horizontal planes...........124

Best axis in three-dimensional space for 161 afferents.............125

Response versus level functions and phase angle versus level functions.............................................. 126

Graphic representations of the model fitting different DRPshapes...136 Example of a unit (pp18) fitted with a pure cosine and the modified cosine fit. ...

Example of a unit (tt7) fitted with a pure cosine and the modified cosine fit.....................................................138

Example of a non-directional unit (ff2) fitted with a pure cosine and the modified cosine fit........................................139 
Figure 4.5 Maximum Z-values obtained from the modified cosine fit plotted as a function of stimulus level.........................................140

Figure 4.6 Mean error plotted for DRPs with maximum Z-values occurring at certain bins.....................................................141

Figure 4.7 Distortion factor of the modified cosine fit plotted as a function of maximum response strength.....................................142

Appendix

Figure A1 Significance of phase locking among primary afferents innervating the saccule or lagena in Acipenser fulvescens.........................162

Figure A2 Distribution of best frequencies across different species..............164

Figure A3 Example of a non-responsive unit.................................165 


\section{CHAPTER I}

\section{Introduction to the Dissertation}

\section{Overview}

Acoustic environments are complex and sounds from several sources often converge on the listener at once. Since animals must be able to behave appropriately toward all relevant objects and events within their environment, their auditory system should be able to assign all acoustic components to the appropriate sources. This, ability, known as stream segregation, has been demonstrated in humans (Bregman 1990), Sturnus vulgaris (European starling; Hulse et al. 1997), and Carassius auratus (goldfish; Fay 1998, 2000). Auditory stream segregation is a fundamental part of a broader concept, auditory scene analysis, which is the parsing of the acoustic environment into separate coherent patterns that are interpreted as individual sources (biotic and abiotic) analogous to the visual scene. A component of source segregation is sound source location. Sound source location gives the scene a spatial dimension.

After the process of stream segregation takes place (providing information about individual sources) and sources are identified and localized an animal is then able to make a decision and behave appropriately (e.g., go towards a potential resource, flee from a potential danger, respond to possible vocalizations, or show no reaction towards a neutral source).

Stream segregation (as part of auditory scene analysis) is thus considered to be the most fundamental function of all auditory systems and probably constitutes the 
major selective pressure shaping the evolution of the sense of hearing throughout vertebrate history (Fay and Popper 2000, Lewis and Fay 2004). As a consequence, those functions of vertebrate hearing that promote stream segregation are likely to have evolved very early in vertebrate history including in the "ancient" vertebrates (cartilaginous fishes and non-teleost bony fishes, Fig. 1).

The term "ancestral"" used throughout the dissertation refers to a group that occupies a basal position in the cladogram of bony fishes (Fig. 1). "Basal" in the phylogenetic context of bony fishes means a closer resemblance of primitive groups with the ancestors of bony fishes. In contrast, "advanced"1 refers to a group of bony fishes (the teleosts) that has less resemblance with the common ancestors as compared to ancestral groups (Liem et al. 2001).

Important components of auditory stream segregation are sound source location and spectral analysis. The major goal of the dissertation is to get a better understanding of the coding strategies for sound source location and frequency analysis, important components of auditory stream segregation, of a non-teleost (ancestral) bony fish, the sturgeon, Acipenser fulvescens (lake sturgeon), as the study species. The results will be compared with data in the literature on teleosts and other vertebrates.

Sturgeons are of general interest for evolutionary studies because of their position within the systematic position of vertebrates (Fig. 1), their large fossil record,

\footnotetext{
${ }^{1}$ The terms ancestral versus advanced (or derived) are meant in a relative sense. For example the presence of mammary glands is ancestral when considering only apes, but advanced when considering all of the Mammalia. Another example is the Weberian apparatus in Otophysans - an ancestral characteristic when considering only Cyprinidae, but advanced when considering Otophysans.
} 
and their diversity (they comprise the largest number of species among ancestral fishes). There are only a few studies on the sensory biology of sturgeons, and nothing is known about their hearing (the same lack of data in hearing applies to the other groups of non-teleost bony fishes). In addition, sturgeons are of great interest because they are increasingly threatened due to commercial exploitation (e.g. over-fishing for caviar production), and because of increased destruction of their spawning habitats due to industrialization. These problems have stimulated research on the life history of sturgeons (migration pattern, reproduction, sensory biology) in order to gather a better understanding of their biology for conservation. ${ }^{2}$

\section{Background}

\section{Significance of spectral analysis and sound source location}

The concept of auditory scene analysis was first described by Bregman (1990) based on experiments with human listeners. Humans exposed to multiple audible sources were able to segregate acoustic components that belonged to one source from components that belonged to other sources. Given this ability, it was possible for humans to determine and discriminate between different sources. The selective advantage arising form this sensory function lies in the ability for an animal to react appropriately when exposed to a complex source array (e.g. for predator and prey detection).

\footnotetext{
${ }^{2}$ A book related to this topic was released in 2002 by Inga Saffron: "Caviar: The strange history and uncertain future of the world's most coveted delicacy," published by Broadway books.
} 
Psychophysical experiments in humans have shown which features of sounds the auditory brain uses to sort sounds. For periodic sounds the physical components were, for instance, harmonicity, ${ }^{3}$ common onset time, common amplitude modulation pattern, common frequency modulation pattern, and common interaural time difference (Lewis and Fay 2004). To utilize these features, the auditory nervous system must decompose monaural input (from many sources) into spectral components. Thus, spectral analysis, the break down of a complex sound into its frequency components, is important for source segregation and identification of sources and is a major topic of investigation of the dissertation with a focus on the auditory periphery.

Behavioral experiments have shown that $C$. auratus is able to segregate simultaneous sources based on differences between source spectra and overall temporal envelope (Fay 1998, 2000). Electrophysiological investigations of frequency tuning in the auditory system showed spectral analysis on the peripheral and central level (e.g. Furukawa et al. 1967, Fay 1978a, Fay and Ream 1986; see following section for more details). Whereas auditory nerve fibers were broadly tuned, sharper tuning could be found in the torus semicircularis (midbrain) of C. auratus (e.g. Lu and Fay 1993) and may explain the behavioral capacities for frequency analysis revealed in psychophysical studies (e.g., Fay and Coombs 1983, reviewed by Fay 1988). Spectral analysis is a common characteristic of vertebrate auditory systems including teleosts. Thus, it is hypothesized that spectral analysis exists in ancient bony fishes as well.

\footnotetext{
${ }^{3}$ Frequency components of a sound that are harmonically related. This means that the component frequencies of a complex tone are integer multiples of a common fundamental frequency.
} 
The ability to localize a sound source is also considered to be an important part of source segregation and auditory scene analysis and has been found in all vertebrate taxa investigated (e.g. Schuijf 1975, Konishi 1973, Fay and Feng 1987, Bregman 2008). The ears of terrestrial vertebrates are sensitive to sound pressure, which is a scalar quantity. In such a system, directional information cannot be determined via a single pressure detector. Thus, directional hearing in terrestrial vertebrates is based on the central computation of differences in sound intensity, phase, and time of arrival between the two ears or via pressure difference receivers (Michelsen and Larsen 2007). In aquatic environments, the higher impedance of water results in higher propagation velocities (about 4.5 times higher than in air) of sound waves, reducing the differences in arrival time and phase at the two ears of a fish. In addition, the small distance between ears relative to the long wavelength of sounds in water makes it unlikely that fish use interaural intensity difference cues (van Bergeijk 1964, 1967).

This insight into physical constraints for sound source location under water initially created a debate as to whether fish can localize sound because it was assumed that fish ears function as sound pressure receivers (v. Bergeijk 1964). In addition, behavioral experiments on sound source location in fish differed in their conclusions, therefore leading to controversy about the general capability of fish to locate a sound source (e.g., von Frisch and Dijkgraf 1935, Kleerkoper and Chagnon 1954, Myrberg et al. 1969, Schuijf et al. 1972, Popper et al. 1973; see also reviews by Popper et al. 2003, Fay 2005, Sand and Bleckmann 2008). In contrast to behavioral experiments, studies on the mechanisms possibly underlying sound source location in fish provided 
less ambiguous insights (Hawkins and Horner 1981, Fay 1984, Fay and Edds-Walton 1997a, Lu et al. 1998, Lu et al. 2003, 2004).

The current understanding on the peripheral mechanisms for sound source location in fish is now based on the notion that fish ears are mainly adapted to detect the particle motion of sound (instead of pressure) functioning as inertial accelerometers (de Vries 1950; see more detail on the structure of the ear and sound detection in fish below). Particle motion is a vector quantity and potentially carries information about the magnitude and axis of a sound source (Rogers and Cox 1988). Thus, for simple monopole sound sources (e.g., a pulsating swim bladder), fish are able to extract the axis running through a sound source at their periphery by sensing the particle motion component of the sound ("vector theory," Schuijf and Buwalda 1975).

Physiological studies of the mechanisms of sound source location have been conducted only in a few species: Gadus morhua (Atlantic cod; Hawkins and Horner 1981), C. auratus (goldfish; e.g., Fay 1984), Opsanus tau (oyster toadfish; e.g., Fay and Edds-Walton 1997a), Porichthys notatus (plainfin midshipman; Weeg et al. 2002), and Dormitator latifrons (sleeper goby; e.g., Lu et al. 1998). All those studies have been conducted in teleosts. In this dissertation, the peripheral mechanisms for sound source location are explored using a non-teleost bony fish for the first time.

\section{Peripheral auditory structures in teleost and non-teleost bony fishes}

The inner ear in bony fishes and elasmobranchs includes three semicircular canals with their associated cristae ampullaris and three otolith organs, the saccule, utricle, and lagena (e.g., Fig. 1.2). Some species of fish, and all elasmobranchs, have 
a seventh end organ, the macula neglecta (Retzius 1881, Corwin 1977). The ear of lampreys possesses only two semicircular canals and one sensory epithelium called the macula communis (Löwenstein et al. 1964).

Each of the otolith organs of all fishes has a sensory epithelium (macula) in close contact with a calcium carbonate mass. In teleosts, the crystals of this mass are fused into a solid structure, the otolith. In non-teleost bony fishes, cartilaginous fishes, and lampreys (and all tetrapods), the mass is composed of crystals that are embedded in a gelatinous matrix (Calström 1963, Lychakov 1995). The functional role of the different forms of calcium carbonate is not known.

The epithelium contains mechanosensory hair cells and supporting cells. Each hair cell possesses an apically located ciliary bundle that contains a large number of stereocilia (e.g., 50 to 70 in Polypterus bichir, Nile bichir, Popper 1978; more than 120 in Protopterus sp., African lungfish, Platt et al. 2004) and a single longer kinocilium (which is a true cilium with a $9+2$ filament pattern). The stereocilia are graded in size, with the longest lying closest to the kinocilium. Hair cell stimulation results from relative motion between the epithelium and the otolith or otoconia, which causes shearing of the apical ciliary bundles (Hudspeth et al. 1977). All three otolith organs in fish are possibly involved in sound detection, and each end organ may be sensitive to different frequency ranges of detection, and/or perhaps to sound from different directions (Popper and Platt 1993, Lu et al. 1998).

Hair cells form orientation groups (each with a different orientation) in which the ciliary bundles of each hair cell point approximately towards the same direction (Fig. 1.3: arrows demonstrate hair cell orientation with the tip of the arrow indicating 
the position of the kinocilium of the hair cells). In most groups of teleost fishes the saccule has four different groups (Fig. 1.3A) that are oriented in a dorsal, ventral, rostral, and caudal axis respectively.

The developmental origin of this orientation pattern of the fish-saccule may, however, be different in teleost and non-teleost bony fishes (Popper 1978). In the primitive bony fish species Lepisosteus osseus (longnose gar), Amia calva (bowfin), P. bichir, and Scaphyrinchus platorynchus (shovelnose sturgeon), ciliary bundles are divided into two distinct groups according to their dorsal and ventral orientation. The rostral end of the saccular macula in these species curves such that the ciliary bundles in that region become oriented horizontally on the animal's rostro-caudal axis (Popper 1978, Popper and Northcutt 1983, Matthiesen and Popper 1987, Platt et al. 2004) (Fig. 1.3B). This non-teleost saccular hair cell pattern resembles the saccular pattern of elasmobranchs (e.g. Corwin 1977), lungfishes (Platt et al. 2004), and of tetrapods (Spoendlin 1964). The basic hair cell orientation pattern of the lagena and utricle of non-teleost bony fishes is similar to that of teleosts.

\section{Sound detection mechanisms in fishes}

Sound is propagated in all media as longitudinal waves carried by pressure fluctuations and particle motions (Rogers and Cox 1988). Particle motions occur in the acoustic "near field" and "far field" of the sound-producing source (van Bergeijk 1967, Kalmijn 1988, Rogers and Cox 1988). Near field particle motions are hydrodynamic flows that are measured near vibrating sources, and their amplitudes attenuate rapidly with distance from the source. In the far field, acoustic pressure and 
the associated acoustic particle motion generated by vibrating objects falls off less steeply with distance than does the amplitude of near field particle motion.

Detection of acoustic pressure requires, in all fish species studied, the presence of a gas bubble closely associated with the ear. Sound pressure fluctuations produce a volume change in gas bubbles. The change in volume in turn causes sound energy to be re-radiated as particle motion that can be detected by the ear. This type of stimulation is called indirect since the gas bubble, for instance the swim bladder, serves as a secondary sound source stimulating the ear (Fay and Popper 1974, 1975, Popper et al. 1988).

In the direct method, particle displacement components of sound moves the fish's body (together with all sensory epithelia) since it has about the same density as water. The density of otoliths differs from the density of the fish body and hence the otoliths move at a different amplitude and phase than the epithelia (and the rest of the fish) when exposed to the particle motion component of sound. The relative motion between otolith and hair cells of the epithelium causes sharing of the ciliary bundles, and hence stimulation of the sensory hair cell (as mentioned above; Popper et al. 1988). In this way, the otolith organs act like inertial accelerometers (de Vries 1950).

Fishes having close contact between the swim bladder (or any other gas-filled structure and the ear) often have a wider hearing range and greater sound-pressure sensitivity than fishes that do not have the two organs in close proximity (although there are exceptions, see for example Coombs and Popper 1982). Based on this proximity of the swim bladder or other gas bubbles to the inner ear, or special connections between inner ear and swim bladder, fishes are typically categorized as 
hearing non-specialists (generalists) or specialists (Popper and Platt 1993). C. auratus is a good example of a hearing specialist and hears frequencies up to $3000 \mathrm{~Hz}$ or even higher (e.g., Enger 1966, Jacobs and Tavolga 1967, Fay 1969). An example of a hearing non-specialist, whose swim bladder is not in close proximity with the ear, is O. tau, which only detects sound up to about $600 \mathrm{~Hz}$ (Fish et al. 1972). Among both specialists and non-specialists, there is generally a considerable interspecific variation in bandwidth and absolute sensitivity. There are studies that have also shown an influence of the swim bladder on hearing sensitivity and frequency range in hearing non-specialists, making the distinction between hearing specialists or non-specialists less clear. For instance, G. morhua possesses a swim bladder, of which the anterior part is in close proximity to the ear and the amplified swim bladder motions in the sound field are conducted through ordinary tissue (no specialized pathways like bones) to the ear. Deflating the swim bladder reduced the hearing sensitivity and shifted the upper frequency limit toward lower frequencies (Sand and Enger 1973). The gas filled bladder therefore acted like a secondary near-field source with particle motions that would decay with the square of the distance to the bladder (in water). Another study in Anguilla anguilla (European eel) showed that even in a species that had the swim bladder located in great distance to the inner ear $(10 \mathrm{~cm}$ for a $50 \mathrm{~cm}$ long eel), the animals showed pressure sensitivity at higher frequencies (Jerko et al. 1989). The author concluded that the transmission-path must have therefore been more efficient than water in A. anguilla.

Auditory specializations, similar to the structures in teleosts, have not been observed in a non-teleost bony fish. A. fulvescens possesses a swim bladder that is 
located in some distance from the ear, however it is not known to what extend sound energy (sound pressure) is transmitted to the ear via the swim bladder.

The hearing capabilities of non-teleost bony fishes are relatively unknown except for recordings of auditory evoked potentials (AEP, formerly called auditory brainstem responses, ABR) in A. fulvescens. The study showed that A. fulvescens could detect frequencies up to $2000 \mathrm{~Hz}$ (but see chapters and discussion of the dissertation on this matter) with lowest thresholds evoking a response between 100 and $300 \mathrm{~Hz}$ (Meyer et al. 2002).

\section{Frequency tuning and directional selectivity of eighth nerve fibers in fish}

In the past, one set of studies on response properties of eighth nerve afferents in fish was directed primarily at understanding of temporal response pattern and tuning. Most of these studies used an underwater loudspeaker to provide acoustic stimuli (Furukawa and Ishii 1967, Fay 1978a, 1978b, Moeng and Popper 1984, Fay and Ream 1986, Lu and Fay 1996, Mc Kibben and Bass 1999, Suzuki et al. 2002). Although a sound generated by a loudspeaker contains pressure and particle motion components, sound pressure was usually calibrated and monitored using a hydrophone. While this may be useful to interpret results from fishes that are responsive to sound pressure, such as hearing specialists, it is less suited for hearing non-specialists, which are mainly responsive to particle motion (or species for which it is unknown to what extent they are pressure sensitive). Thus, a second set of studies had been aimed at exclusively using particle motion for stimulation (Sand 1974, Schuijf 1975, Fay 1984, Fay and Edds-Walton 1997b, Lu et al.1998). For this purpose 
a shaker table was designed to provide linear acceleration of the fish, thereby simulating underwater particle motion (Sand 1974, Fay 1984).

Using either a loudspeaker underwater or a shaker table, frequency tuning and temporal response of primary otolith afferents (mostly saccular afferents) were obtained from several teleost species: C. auratus (reviewed in Popper and Fay 1999), Ictalurus punctatus (catfish, Moeng and Popper 1984), Cottus scorpius (bullhead, Enger 1963), G. morhua (Horner et al. 1981), O. tau (Fay and Edds-Walton 1997b), P. notatus (McKibben and Bass 1999, Weeg et al. 2002), Pollimyrus adspersus (Suzuki et al. 2002), and D. latifrons (Lu et al. 2003, 2004). These investigations generally revealed that afferents expressed great variation in sensitivity, best frequency, and bandwidth (Fay 1981, Fay and Ream1985, Fay and Edds-Walton 1997b, McKibben et al.1999). In addition, frequency selectivity in the auditory periphery of these teleosts appeared to be similar to that found in the auditory receptor organs of other vertebrate classes that have been studied (Popper and Fay 1999). However, unlike in terrestrial vertebrates, these fishes have just a small number of differently tuned channels in the periphery (Fay 1997, Popper and Fay 1999).

All afferents being responsive to sound showed phase locking (Fay 1981, Fay and Edds-Walton 1997b). Phase locking has been hypothesized to play a role in frequency analysis in tetrapods (Wever 1949) and fishes (Fay 1978a). In C. auratus, the temporal error with which saccular afferents synchronized to tones could predict behavioral frequency discrimination acuity (Fay 1978b). Phase locking occurs in low frequency afferents of all vertebrate auditory systems (for frequencies below $\sim 4 \mathrm{kHz}$ 
in most cases) and was therefore considered to be a primitive characteristic of the neural code for hearing (Popper and Fay 1999).

Directional selectivity of eighth nerve afferents was investigated using a shaker table that moved the fish along defined axes through linear acceleration (simulating particle motion as mentioned above). The shaker table developed by Fay (1984) moved the fish along axes in three dimensions. Eighth nerve afferents responded to variations in stimulus axis in a cosine-like manner with best directions that were the result of the hair cell orientations of the epithelium that they innervated and the orientation of the epithelium in space (Fay 1984, Edds-Walton 1997a, Lu et al. 1998, Lu and Popper 2001). Afferents innervating the saccule or lagena showed a preference for the horizontal angle that corresponded to the horizontal orientation of the epithelium (or spatial orientation in azimuth) they innervated (Fay 1984, Lu et al. 1998, Edds-Walton et al. 1999, Lu et al. 2003). This theoretically allows for central comparison of the difference in response magnitudes arising from the right and left saccules or lagenae which in turn could be used to compute the location of a sound source in azimuth with a few ambiguities (Fay 1984, Fay and Edds-Walton 1997a).

The current view on the peripheral physiology of auditory systems in fish is based on a few studies in teleosts. Studies on non-teleosts, and especially non-teleost bony fishes, are important to more clearly determine the functions that are ancestral and those that are derived.

From a functional point of view, the shaker table is a better stimulation method to investigate frequency selectivity for particle motion detectors (and the only method to investigate directionality), yet very few studies have been conducted using 
this method for frequency studies (Fay and Edds-Walton 1997b, Weeg et al. 2002, Lu et al. 2003, 2004).

\section{Systematic position and special features of sturgeons}

Sturgeons belong to the order Acipenseriformes, which, together with the Polypteriformes (bichirs and reedfishes), makes up a group called Chondrostei or "lower Actinopterygian" fishes (Grande and Bemis 1996). The rest of the Actinopterygian fishes, called "higher Actinopterygians" comprise the orders Ginglymodi (gars and their close relatives), Halecomorphi (bowfins or Amiidae and their close relatives), and teleosts (see Fig.1.1). These three "higher" actinopterygian groups are also collectively referred to as Neopterygii (Gardiner 1993), implying with this name a more recent origin in the evolution of bony fish than the "lower Actinopterygians."

Acipenseriformes include two fossil families, the Chondrosteidae and the Peipiaosteidae, and two extant families, the Acipenseridae (sturgeons, 25 living species) and Polyodontidae (paddlefishes, two living species). The most plausible place and time of origin of Acipenseriformes is the Triassic (ca. 200 million years ago) of Western Europe since this is consistent with the range of Bigeria, the closest outgroup of Acipenseriformes and the occurrence of Chondrosteidae (Bemis and Kynard 1997). Of the two extant Acipenseriform families, only Polyodontidae had undergone comprehensive phylogenetic studies so far (Grande and Bemis 1991).

Acipenseriformes have been studied in the past for numerous reasons: first, from an evolutionary perspective, comparisons between Acipenseriformes and teleosts provide important insights into which character traits are evolutionary 
ancestral and which are derived (Gardiner 1984). Acipenseriformes contain many living species (27 versus 16 species of Polypteriformes), they have a large fossil record, and they are subject of renewed descriptive and phylogenetic studies (e.g. Grande and Bemis 1991, Findeis 1991, 1993, 1997). Second, the biology of these fishes is of interest to systematists and naturalists because sturgeon are often the largest freshwater animals in their respective fauna. They comprise an unusual mixture of characters (phylogenetic, individual, and ontogenetic variation) and widespread geographical distribution. Third, Acipenseriformes are increasingly threatened in their native ranges (e.g., Birstein 1993), yet they have very important economic importance. The decrease of the population sizes can be traced to three major factors: certain life history characteristics of these fish (slow sexual maturation, females often reproduce only once every few years, and migratory nature), the destruction of spawning habitat by pollution or dams, and over-fishing.

Figure 1.1 shows the relationships of living Polypteridae, Acipenseriformes, Lepisosteidae, Amiidae, and Teleostei. Although sturgeon (Acipenseridae) and paddlefishes (Polypteridae) are often called "living fossils," it does not mean that features present in the living sturgeons and paddlefishes are necessarily ancestral. Such hypothesis must be tested by comparison to other Actinopterygians.

Polypteridae, Acipenseriformes, Lepisosteidae, and Amiidae together contain about 48 living species (versus at least 26,840 living species of teleosts, Nelson. 2006). Of these 48 species, 25 are sturgeons. Sturgeons also show the largest 
biogeographical range of any living clade ${ }^{4}$ of non-teleostean Actinoptergians.

Because of their diversity and phylogenetic position as a basal group within

Actinopterygians, sturgeons, or more generally, Acipenseriformes, are considered to be essential for comparative studies within extant and fossil Actinoptergygians in the evolutionary literature (Bemis et al. 1997).

Because of the importance of sturgeon in understanding the evolution of fishes, it was decided to use species of this group to focus on for this project studying the evolution of hearing mechanisms.

\section{Significance}

Over the last two decades, studies have shown that the auditory systems of fishes (mainly on teleosts) are capable of performing complex signal analyses comparable to those performed by mammals. In spite of the diversity in peripheral and central auditory structures of vertebrates, hearing systems may have evolved to perform certain basic tasks, such as sound source location and spectral analysis which are important for sound source segregation and auditory scene analysis (Fay and Popper 2000).

Neurophysiological data on ancestral fishes with a primary focus on sturgeon might help to support the hypothesis that basic hearing functions are more conservative than the structures that underlie them. Sturgeons have never been investigated in such detail before. Thus, this study not only will contribute to the

\footnotetext{
${ }^{4}$ A clade is a monophyletic group of organisms that includes all known descendants of a hypothetical ancestor and no other members (e.g. the family Acipenseridae includes all sturgeon species, but no paddlefishes, Liem et al. 2001).
} 
understanding of hearing in fish (specifically the coding strategies for spectral analysis and sound source location in fish) but also of hearing among vertebrates in general. In addition, this is the first study on sensory coding in a non-teleost bony fish.

\section{Introductory method section}

This section provides a description of a few illustrations (Figs. 1.4 to 1.7) that are considered to complement or emphasize certain aspects of the methods described in the experimental chapters two and three. For details, please refer to the method sections described in those chapters.

Figure 1.4 illustrates the three-dimensional shaker table (developed by Fay 1984) and used in this dissertation for stimulation of the ear and auditory/vestibular periphery of A. fulvescens. The photograph shows the top view of the setup with a juvenile A. fulvescens (between one and two years old) in the dish. The animal was tightly connected to the stimulus dish at its head via a head holder specifically manufactured for A. fulvescens (notice the three screws of the head holder at the top of the skull, Fig. 1.4 top). The head holder contained a metallic tube that provided the fish with constant water flow (artificial respiration) during the experiment. The screws of the head holder pressed the head from the top against the tube inserted into the fish's mouth. The tube in return was tightly screwed to the stimulus dish. This arrangement guaranteed that any movement of the dish was translated to the animal and that the (immobilized) animal was sufficiently respirated throughout the duration of the entire experiment. 
The stimulus dish was driven by two pairs of shakers mounted at the sides of the dish and in front back direction (with respect to the orientation of the animal). The relative output of the front-back shaker pair versus the side-side shaker pair created motions along distinct axes in the horizontal plane (Fig. 1.4 top and bottom a). A fifth shaker was mounted to the bottom center of the dish creating up and down motion (Fig. 1.4, bottom b). The combined output of the front-back shaker pair and the vertical shaker created motions along distinct axes in the vertical (or midsaggital) plane of the fish. Six axes in both, the horizontal and vertical planes were used for stimulation to determine directional response selectivity in eighth nerve afferents in A. fulvescens (Fig. 1.4 bottom c, d).

The signals to the shaker system were $500 \mathrm{~ms}$ long digitally synthesized sinusoids. The frequency of the sinusoids was modified to test frequency selectivity of afferents in A. fulvescens. Directional selectivity was tested at the best frequency (BF) of most afferents $(100 \mathrm{~Hz})$, and frequency selectivity was investigated at the best axis (BA) of most afferents $\left(90^{\circ}\right)$. The dish movement was calibrated for each experiment before and after the fish was positioned in the stimulus dish by use of three orthogonally oriented accelerometers mounted on the side to side, front back, and vertical axes of the dish (Fig. 1.4 bottom a, b).

Recordings were made from the right posterior ramus of the eighth nerve, which contains fibers that innervate the saccule, lagena, macula neglecta, and the posterior crista (Fig. 1.5). The access to the nerve was only possible by retracting the brain slowly to the side. The electrode was placed at the location on the posterior ramus that was located most proximal to the brain. It was impossible to access any 
distal portions of this ramus for recordings (for instance to access the small branch that just innervates the lagena) due to the tight connection of the nerve with the pouch containing the saccule and lagena without severely damaging those epithelia.

Action potentials were recorded extracellularly from single afferent fibers (see example shown in Fig. 1.6) using 2M NaCl-filled glass pipettes with resistances between 20 and $50 \mathrm{M} \Omega$. Experience showed that in order to obtain directional data of good quality (resulting in cosine like response profiles) a very good signal noise ratio (avoiding the chance of recording multiple units at the same time) was essential.

From the spike times recorded during stimulation, period histograms (PHs) were formed and the coefficient of synchronization $\mathrm{R}$ was calculated to measure the degree of phase locking. For each R-value, a Z-value was computed from $Z=R^{2} N_{s}$ where $\mathrm{N}_{\mathrm{s}}$ represented the number of spikes. A Z-value of 20 for instance represents a chance of 0.001 that the spike times recorded were random and not stimulus coupled (Rayleigh statistic). Since afferents strongly phase-coupled to the stimulus waveform and since most units were spontaneously active, the Z-value was used as a measure of response strength for the data-analysis.

Figure 1.7 illustrates the directional selectivity of one afferent. The response strength $\mathrm{Z}$ is plotted as a function of stimulus angle for stimuli in the vertical plane forming a directional response profile (DRP; Fig. 1.7 top graph). For each plot, the response data at the six angles were plotted twice, once at the nominal axis angle and again at the angle plus $180^{\circ}$. For the vertical plane DRP measured at $20 \mathrm{~dB}$ re $1 \mathrm{~nm}$ (see the thicker line at $20 \mathrm{~dB}$ ), the peristimulus time histogram (PSTH) and the $\mathrm{PH}$ for two data points are shown to illustrate how the response strength (spike rate, see 
raster plot and PSTH) and degree of phase locking (see $\mathrm{PH}$ ) changed as a function of stimulus axes (comparing the best response at $90^{\circ}$ with lowest response at $0^{\circ}$ ).

With increasing stimulus intensities (starting at $20 \mathrm{~dB}$ re $1 \mathrm{~nm}$ ) the unit became responsive to horizontal plane stimuli (Fig. 1.7 bottom graph). The best axis for horizontal plane stimuli was $90^{\circ}$ (front back) and the lowest response occurred at $0^{\circ}$ (side to side motion). As will be discussed later in chapter three, most units having their best axis (BA) at $90^{\circ}$ vertical, would not respond at all to horizontal plane stimuli. However, for the purpose of illustrating how the directional data were analyzed in terms of responses in both planes, this example was chosen.

\section{Chapter overview}

This dissertation investigates the response properties of primary eighth nerve afferents to acoustic particle motion in a non-teleost bony fish, Acipenser fulvescens (the lake sturgeon). Chapter 2 focuses on a detailed analysis of frequency selectivity, intensity responses, and temporal response pattern. The results contribute to the discussion of coding strategies by the auditory/vestibular periphery for frequency and intensity of sound in fish and the evolution of auditory coding in fish and other vertebrates. In addition, Chapter 1 provides information on the frequency range and the intensity of sound that the auditory/ vestibular periphery in a non-teleost bony fish detects.

Chapter 3 investigates the directional selectivity of primary eighth nerve afferents in A. fulvescens. Responses of eighth nerve afferents to directional stimuli provided by movement of the fish along defined axes in three dimensions are tested. 
Mechanisms of encoding sound direction are discussed for A. fulvescens and compared to studies in teleosts. The directional response characteristics of eighth nerve afferents were fitted with a model to better categorize the shape of the directional response profile and its dependency on level in Chapter 4.

In Chapter 5, all results from Chapters 2, 3, and 4 are taken together and placed into a broader evolutionary and functional context of hearing in vertebrates. 


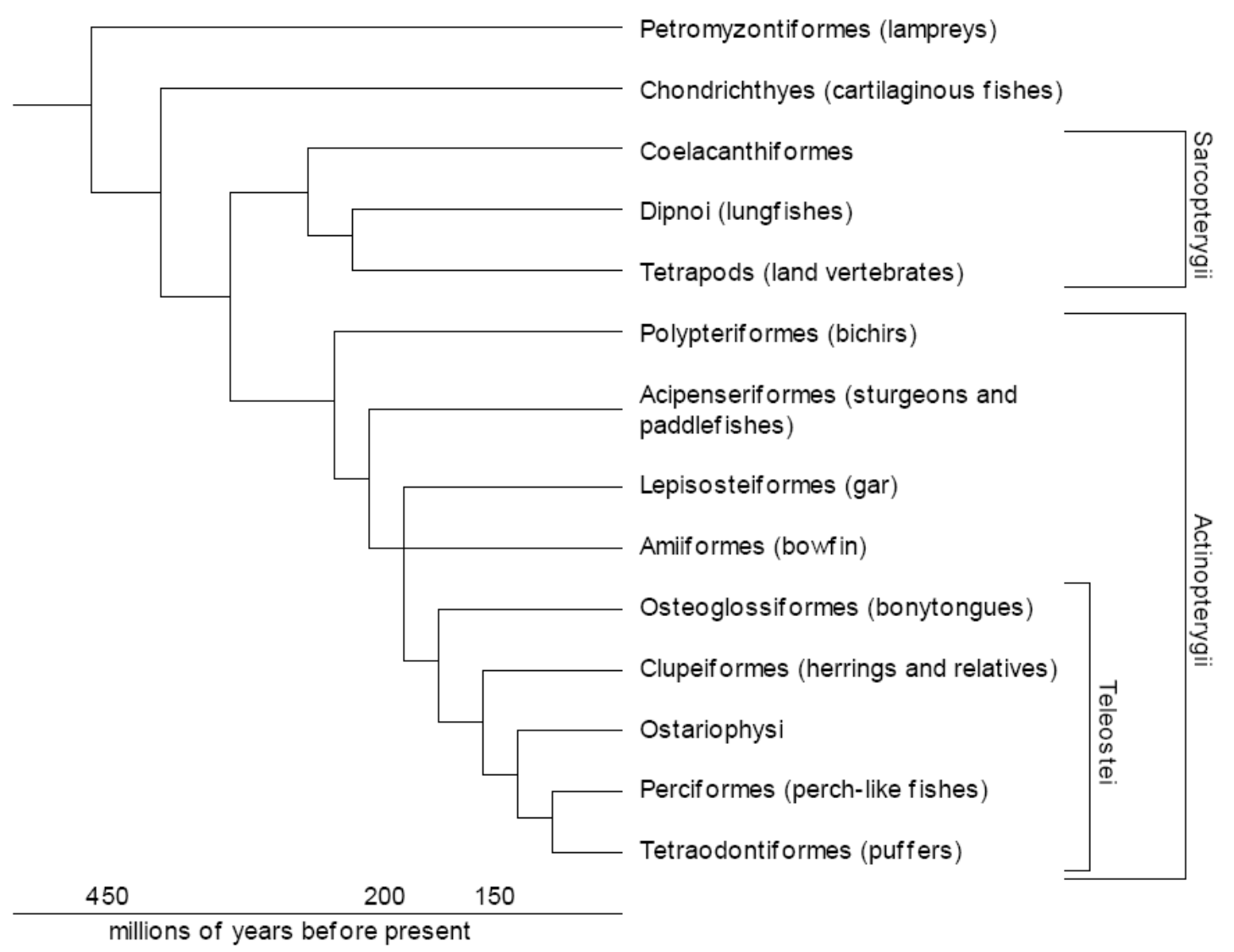

Fig. 1.1: Phylogenetic relationship of extant vertebrate lineages. Modified after Coffin 2005. Tree is mostly based on Nelson 2006. 


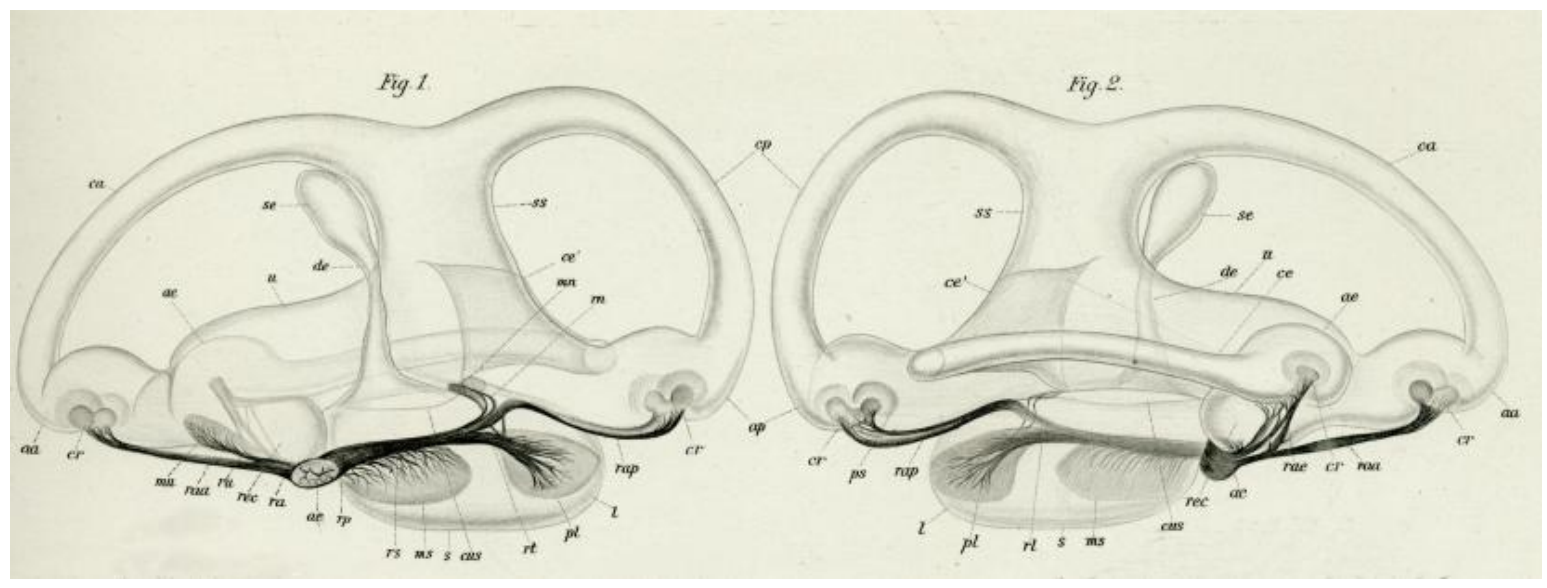

Fig. 1.2: the right ear of Acipenser sturio (European sea sturgeon), original drawings by Retzius (1881).

Abbreviations: $\mathrm{u}$ - utriculus, ss - sinus utriculus superior, rec - recessus utriculi, Aa - ampulla anterior, ae ampulla externa, ap - ampulla posterior, ca - canalis $\mathrm{m}$. anterior, ce - canalis $\mathrm{m}$. externus, ep - canalis $\mathrm{m}$. posterior, $\mathrm{s}$ - sacculus, cus - canalis utriculo-saccularis, de - ductus endolymphaticus, 1 - lagena chochleae, $\mathrm{mu}$ - macula ac. recessus utriculi, cr - crista ac. amp., ms - macula ac. sacculi, mn - macula ac. neglecta, $\mathrm{pl}$ papilla ac. lagenae, ac - acusticus, ra - ramus anterior acustici, rp - ramus posterior acustici, ru - ramus rec. utriculi, raa - ramus amp. anterioris, rae - ramulus amp. anterioris, rae - ramulus amp. externae, rap ramulus amp. posterioris, $\mathrm{rs}$ - ramulus sacculi, $\mathrm{rn}$ - ramulus neglectus, $\mathrm{rl}$ - ramulus lagenae 


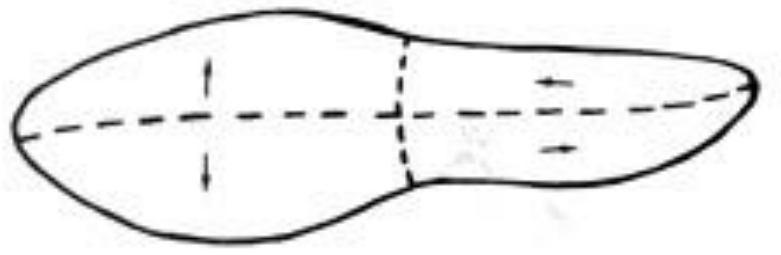

D

A. Sardinella marquesensis

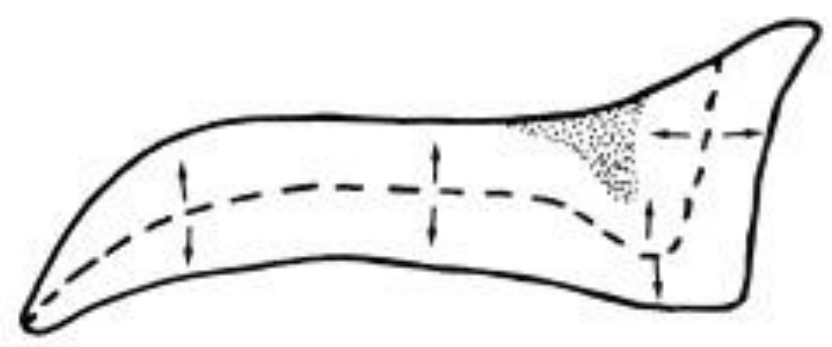

B. Scaphirhynchus platorynchus

Fig. 1.3: Saccular maculae of a teleost fish species, the sardine (A) and a non-teleost bony fish, the shovel nose sturgeon $(\mathbf{B})$ - from Platt and Popper with permission (1981). Arrows demonstrate hair cell orientations with the tip of the arrow indicating the position of the kinocilium of the hair cells. The dashed line represents a boundary line between the regions with opposing hair cell orientations. 


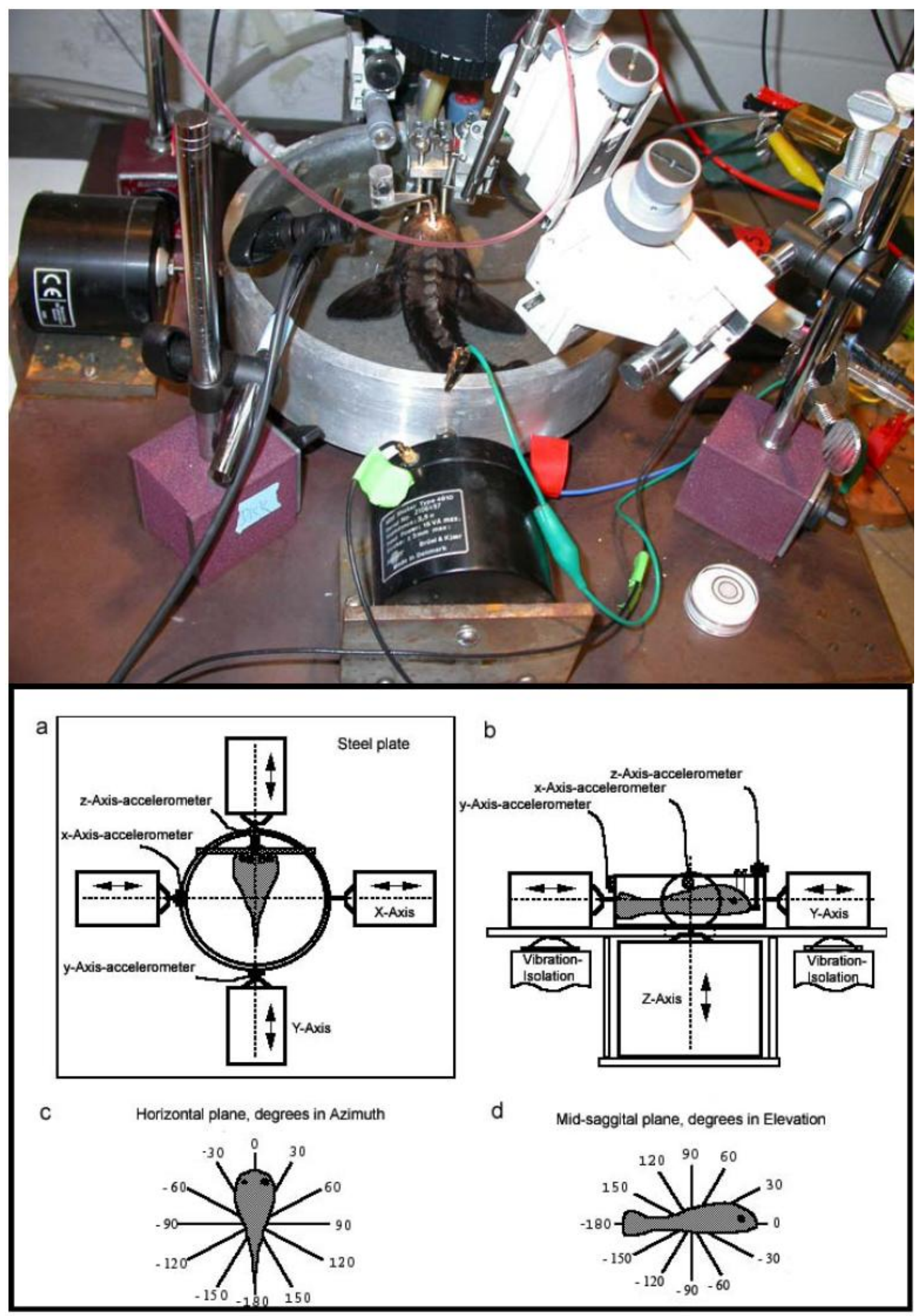


Fig. 1.4: Photograph (top) and schematic representation of the three-dimensional shaker system (bottom; modified from Fay and Edds-Walton 1997a with permission). Schematic diagram: a: top view showing two pairs of shakers for side to side (x-axis) and front back stimulation (y-axis) in the horizontal plane. Opposing shakers are $180^{\circ}$ out of phase, creating a push-pull action on the dish. The top view corresponds to the view shown in the photograph (top) with juvenile Acipenser fulvescens tightly mounted to the stimulus dish by its head. b: side view of the setup illustrating the vertical (z-axis), and vibration isolation of the table. c: six stimulus axes in the horizontal plane. $d$ : six stimulus axes in the vertical/midsaggital plane. 


\section{Recording location}

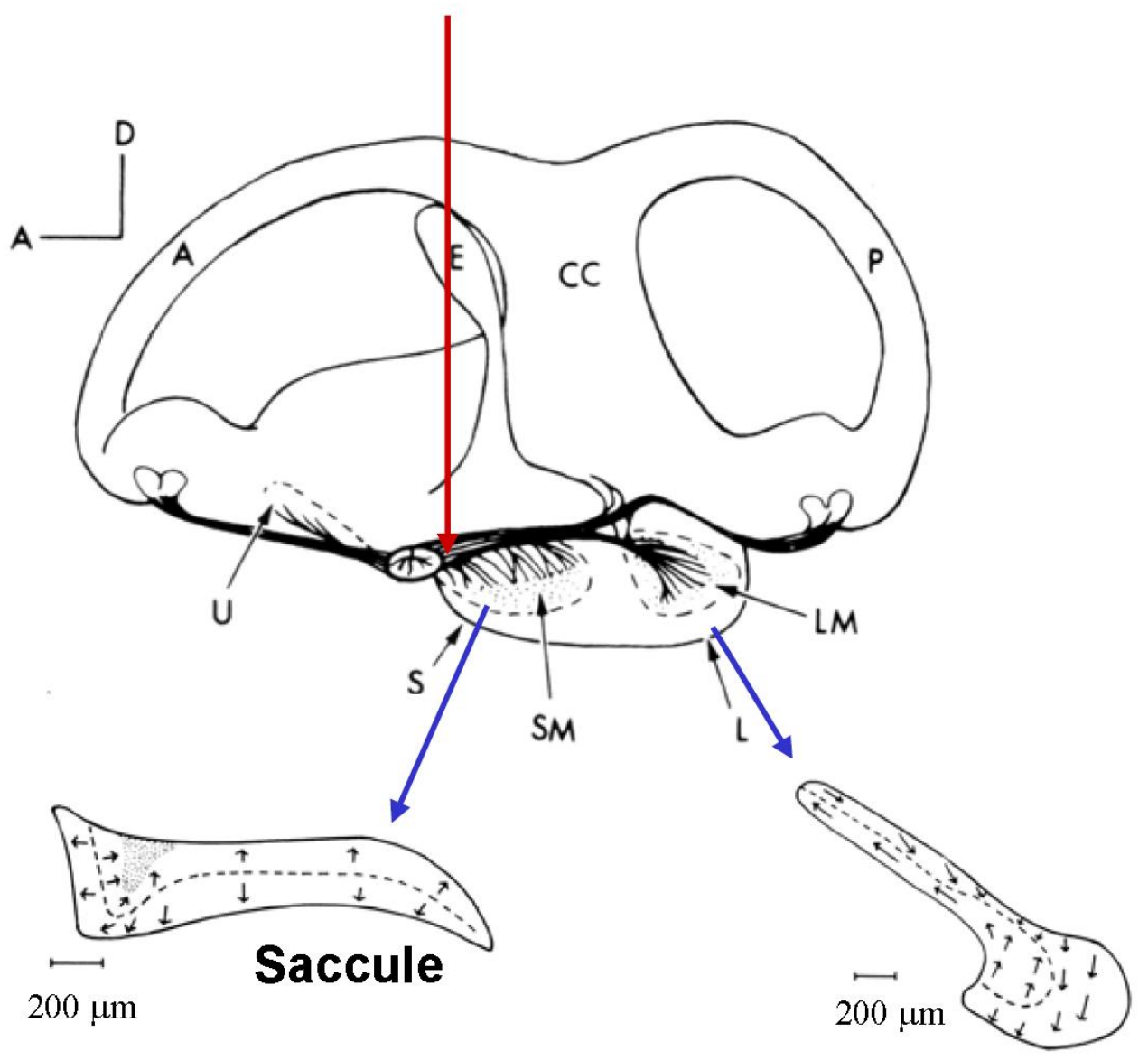

Lagena

Fig. 1.5: Right ear of Acipenser sturio (modified from Popper 1978 with permission) looking very similar to the ear of A. fulvescens. The red arrow indicates the recording location on the right posterior ramus of the eighth nerve. Abbreviations: A - anterior semicircular canal, CC - crus commune, E - Endolymphatic duct, S - Saccule, SM Saccular macula, U - Utricle. 

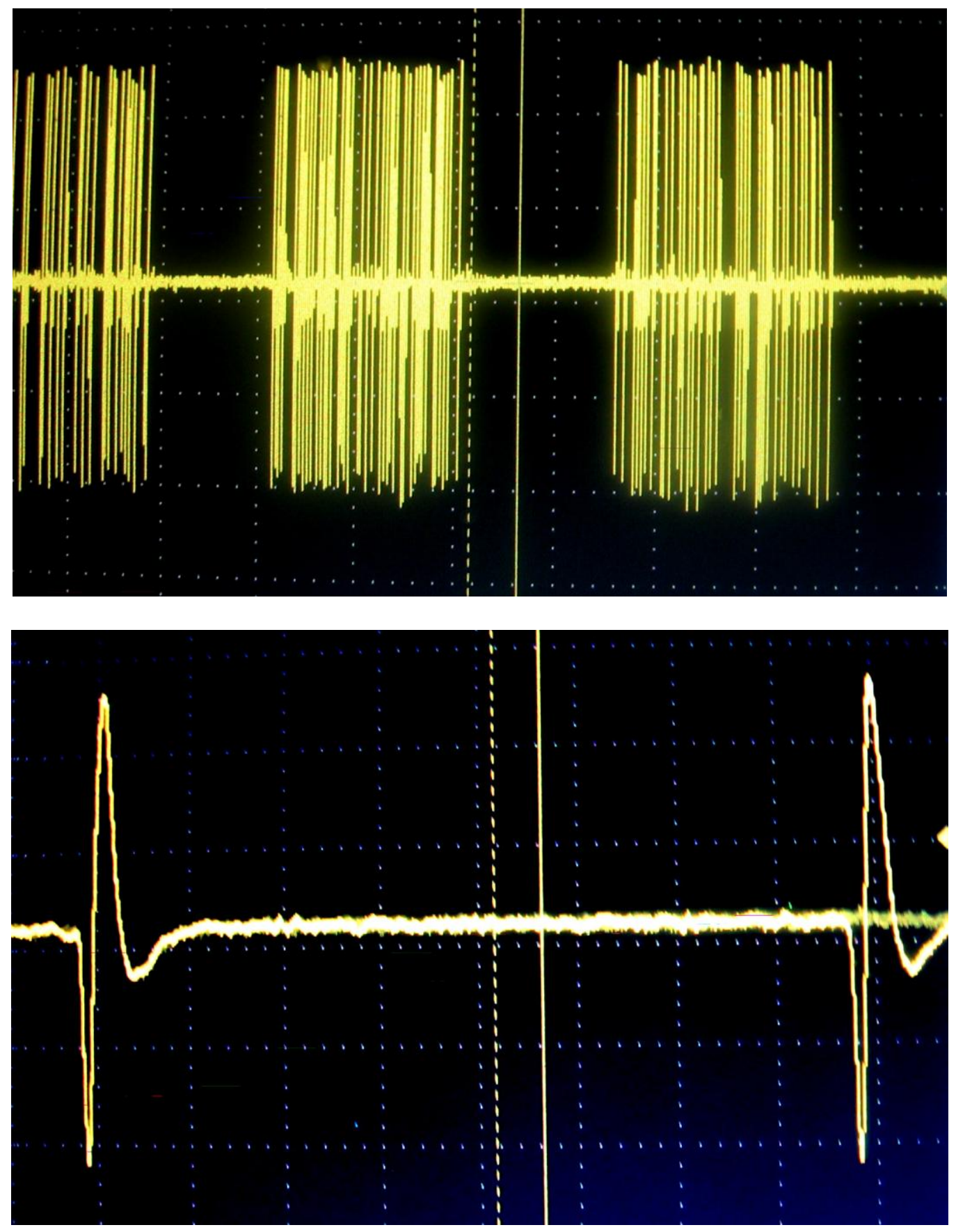

Fig. 1.6: Action potential traces of a single unit. This unit was not spontaneously active and highly phase locked to the stimulus waveform. Top: Time per square (xaxis): $200 \mathrm{~ms}$. Amplitude per square (y-axis): oscilloscope reading $=500 \mathrm{mV}$ corresponding to $500 \mu \mathrm{V}$ actual amplitude. Bottom: Zoomed into AP trace on top. Amplitude per square: $500 \mu \mathrm{V}$. Time per square: $4 \mathrm{~ms}$. 

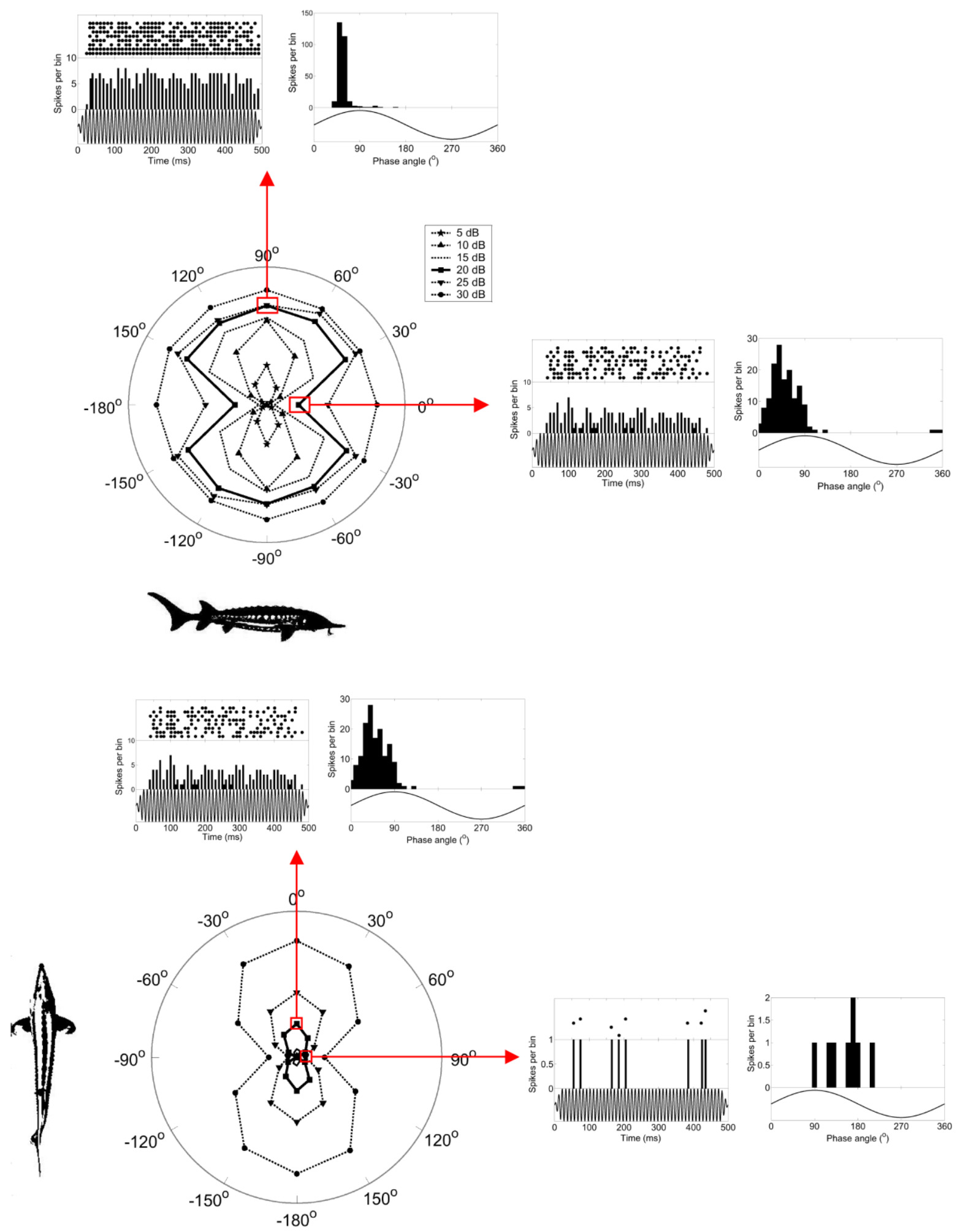
Fig. 1.7: Vertical plane directional response profiles (DRPs, top) and horizontal plane DRPs (bottom) of the same unit are plotted at different levels (see legend next to vertical plane DRP, top) of the same unit. For each DRP the Z-value is plotted as a function of stimulus angle.

Peristimulus time histograms and period histograms are shown for $90^{\circ}$ vertical, $0^{\circ}$ vertical, $0^{\circ}$ horizontal, and $90^{\circ}$ horizontal at corresponding $\mathrm{Z}$ values (at $20 \mathrm{~dB}$ re $1 \mathrm{~nm}$ ) in the DRPs. 


\section{CHAPTER II}

\section{Frequency tuning and intensity coding of sound in the auditory periphery of the lake sturgeon, Acipenser fulvescens}

\section{Introduction}

The decomposition of sound into its frequency components through spectral analysis is common in all vertebrate auditory systems (Lewis and Fay 2004). Spectral analysis of a sound signal reveals which frequencies are contained within a signal and the relative proportions (amplitudes) of the frequencies to one another, thereby characterizing the signal's spectrum. Spectral analysis is required for sound source segregation and the identification of sound sources, which are important for auditory scene analysis and communication (Bregman 1990, Fay and Popper 2000, Lewis and Fay 2004). Generally, frequency selectivity (having a set of auditory filters) is also necessary to restrict the ambient frequency range therefore improving signal noise detection (Popper et al. 2002).

Several mechanisms have evolved in the vertebrate auditory periphery to extract frequency information from sound. In the mammalian cochlea, a traveling wave on the basilar membrane performs the primary frequency separation (von Békésy 1960). In land vertebrates (birds, reptiles, mammals) hair cell resonance and micromechanical interactions between a tectorial membrane and hair cells are additional important mechanisms for peripheral frequency selectivity (Lewis and Narins 1999, Gleich and Manley 2000, Manley 2000). While there are differences in 
the origin of frequency selectivity between land vertebrates, common to most is a tonotopic arrangement of hair cells in the auditory periphery (Manley 1986). There is no evidence, however, that fish have such a tonotopic arrangement of hair cells in their auditory periphery, yet afferents innervating otolith organs in the few fish species studied to date are frequency selective (e.g. Fay 1981, Fay and Edds-Walton 1997b, McKibben et al.1999, Lu et al. 2003, 2004).

Most studies on spectral analysis and the underlying peripheral coding mechanisms for frequency analysis in fish have been conducted in teleosts (modern bony fishes), the most studied of which is Carassius auratus (goldfish). Behavioral experiments have shown that $C$. auratus is able to segregate simultaneous sources based on differences between source spectra and overall temporal envelope (Fay 1998, 2000). Moreover, electrophysiological investigations of frequency tuning in the auditory system have demonstrated spectral analysis at the peripheral and central levels. Auditory nerve fibers differ in their best frequencies for sinusoidal stimuli (e.g. Furukawa et al. 1967, Fay 1978a, Fay and Ream 1986). In addition, the peripheral frequency selectivity appears to be enhanced by inhibition in the central auditory system (Lu and Fay 1993, 1996).

While $C$. auratus belongs to the group of teleost fish characterized as hearing specialists since it can detect sound pressure through a swim bladder that is directly coupled to the ear, most fish do not have such specializations (hearing non-specialists, or generalists; although there are exceptions, see Coombs and Popper 1982). However, all fish share a common mechanism for ear stimulation that involves one or more of the otolith organs (saccule, lagena, and utricle), which respond directly, as 
inertial accelerometers, to acoustic particle motion which accelerates the fish's body in a sound field (de Vries 1950, Fay 1997a).

There are a few studies that have tested the responses of eighth nerve afferents in fish to different frequencies and intensities. Those investigations have only been conducted in teleosts including C. auratus (Fay 1984, Meyer et al. 2004), Opsanus tau (oyster toadfish; Fay and Edds-Walton 1997, Edds-Walton and Fay 1999), Porichthys notatus (plainfin midshipman; Weeg et al. 2002), Dormitator latifrons (sleeper goby; Lu et al. 1998, 2003), and Ictalurus punctatus (channel catfish, Moeng and Popper 1984). Recordings from saccular, lagenar, or utricular afferents revealed that there are a small number of discretely tuned peripheral channels in contrast to a more continuous tuning found in many land vertebrates (Fay 1997, reviewed in Popper and Fay 1999, Lu et al. 2003, 2004). Another mode for encoding sound frequencies is the temporal encoding through phase locking of eighth nerve afferents in fish (Fay 1978a) as well as in other vertebrates for frequencies below about $4 \mathrm{kHz}$ (Popper and Fay 1999).

There are, however, no studies on the response properties of eighth nerve afferents (or any behavioral studies such as conducted with C. auratus) in a nonteleost bony fish. Acipenser fulvescens, the lake sturgeon (Rafinesque 1817), belongs to the ray-finned fishes or Actinopterygians. The great majority of Actinopterygians consist of the teleosts (more than 26,000 species, Nelson 2006). In contrast, there are only 48 extant non-teleost Actinopterygians species (Liem et al. 2001). Of these, 25 species are sturgeons (family: Acipenseridae), while two species belong to a sister group, the paddlefishes (family: Polyodontidae). Both Acipenseridae and 
Polyodontidae belong to the order Acipenseriformes which are known to have a very long phylogenetic history dating back about 250 million years (Grande and Bemis 1996). The ancient heritage of the Acipenseriformes, along with their migratory behavior (Kynard 1997) and their substantial economic importance, has stimulated recent interest in their sensory capabilities (e.g., Sillman et al. 2007).

From an evolutionary perspective, studies on non-teleost Actinopterygian groups are important since they provide insight into mechanisms of signal processing in an ancestral group. Moreover, such studies are critical for comparison with teleost studies since they allow one to distinguish which character traits are ancestral and which are derived (Wilez 1981, Northcutt 1985, 1986, Bolker 2004). In addition, physiological studies on hearing in any other ancestral group of fishes (cartilaginous, jawless, or lobe finned fishes) have not been conducted before (except for two studies in sharks, Corwin 1989, Casper and Mann 2007). In summary, by selecting an ancestral bony fish (phylogenetically very different from teleosts), this study seeks to add to the understanding on how fish perform frequency analysis and to the origin of frequency analysis in fish and land vertebrates.

The ear and auditory periphery of A. fulvescens resembles that of hearing nonspecialist teleosts and other non-teleost bony fishes, in that it does not appear to possess any auditory specializations (Popper 1978, Mathiesen and Popper 1987, Popper and Northcutt 1983). The ear contains three otolithic end organs (utricle, saccule, lagena), each of which is in contact with a calcium carbonate mass composed of crystals (otoconia) that are embedded in a gelatinous matrix (Calström 1963, 
Lychakov 1995, Gauldie 1996, Lovell et al. 2005). A. fulvescens has a swim bladder that is not in close proximity to the ear (personal observation).

In this study we investigate the responses of primary eighth nerve afferents (innervating the saccule or lagena) in A. fulvescens with respect to frequency selectivity, intensity responses, and response pattern. An adequate method to stimulate otolith organs used in previous experiments on teleosts is by linear acceleration of the animal, thereby simulating the effect of acoustic particle motion under water (Fay 1984). Since there are no major structural differences between the ear of A. fulvescens and the ears of teleosts, the same stimulation method was used and A. fulvescens was exposed to linear acceleration at different frequencies and intensities. From a functional point of view, the shaker table producing linear acceleration is a better stimulation method (as opposed to stimulation by a loudspeaker and calibration in terms of pressure) to investigate frequency selectivity for particle motion detectors, yet only a few studies have been conducted using this method for frequency studies (e.g. Fay and Edds-Walton 1997b, Weeg et al. 2002, Buchser et al. 2003, Lu et al. 2003, 2004).The data presented here represent the first detailed electrophysiological study investigating sensory coding strategies in a nonteleost bony fish.

\section{Material and Methods}

\section{Animal preparation}

A. fulvescens, 25 to $37 \mathrm{~cm}$ in total length ( 21 to $28 \mathrm{~cm}$ in standard length) were obtained from the Wild Rose fish hatchery of the Wisconsin Department of 
Natural Resources. The fish were between one and two years old and their body masses ranged between 45 and $115 \mathrm{~g}$. They were housed in 45 -gallon tanks containing aerated water filtered by external filters and kept at a temperature between 17 to $18{ }^{\circ} \mathrm{C}$ on a $12 \mathrm{~h}$ light/12 $\mathrm{h}$ dark cycle. Recordings from 21 fish were used. For referral to certain units and experiments (see Results), the convention aa, bb, cc, etc. was used to designate specific animals, and numbers were use to identify units (e.g. “pp9" stands for unit number 9, recorded from animal "pp"). The care and experimental use of A. fulvescens were carried out using protocols approved by the University of Maryland Institutional Animal Care and Use Committee.

A. fulvescens were lightly anesthetized in a bath containing $0.05 \%$ buffered 3 aminobenzoic acid methane-sulfonate salt (Sigma) dissolved in tank water, immobilized with an intramuscular injection of gallamine triethiodide in goldfish Ringer's solution ( $43 \mu \mathrm{g} \mathrm{g}^{-1}$ body weight), and then transferred to a surgical dish. For artificial respiration, aerated water was continuously pumped through the fish's mouth with a flow rate of 100 to $200 \mathrm{ml}$ per minute. To locally anesthetize the surgical area, the skin at the dorsal surface of the head was dabbed with $2.5 \%$ Lidocaine (Astra Chemicals GmbH, Wedel, Germany). An opening into the cranium was made at the level of the entrance of the eighth nerve into the brain, and excess fatty tissue and fluids were aspirated away to reveal the brain surface above the eighth nerve (part of the cerebellum and medulla became exposed during this procedure). The exposed brain was kept constantly moist with a fluoroinert liquid (FC-77, 3M Corp). 
After completion of the surgery, the fish was placed in an aluminum dish $($ diameter $=23 \mathrm{~cm}$, height $=6 \mathrm{~cm})$ filled with water from a filtered water tank reservoir. The cylinder was mounted on a three-dimensional shaker table (described below). A rigid head-holder with a respirator tube especially designed for $A$. fulvescens was used to secure the head of the fish to the cylinder. Aerated tank water (temperature: 17 to $18^{\circ} \mathrm{C}$ ) was pumped through the respirator into the fish's mouth via a Neslab RTE-111 chiller pump. The temperature of the water in the cylinder (corresponding to the temperature of the holding tank of the animal) was kept constant throughout the entire experiment.

In preparation for electrophysiological recordings from the posterior ramus of the eighth nerve (innervating the right ear), the brain was carefully retracted to the left side using a self-made plastic retractor. Special care was taken that semicircular canals and all otolith organs of the ear were not damaged during this delicate process. The water level in the cylindrical dish was just below the surgical opening.

\section{Stimulation system}

The shaker table developed by Fay (1984) uses five mechanical shakers to move the dish. Four shakers (Bruel and Kjaer \# 4810), two mounted in front-back and two mounted in side-to-side position created linear oscillatory motions of the cylindrical stimulus dish, thereby moving the fish along distinct axes in the horizontal plane. A fifth shaker (Bruel and Kjaer \# 4809) was attached via a rod to the bottom

center of the dish and functioned to oscillate the fish vertically. The combined output from the front-back shaker pair and the vertical shaker created motions along distinct axes in the midsaggital plane of the fish. The movement of the fish produced by these 
shakers simulated the effects of particle motion of underwater sound in a natural environment. This stimulus system was used in previous experiments mostly to investigate directional responses in fish (e.g. Fay 1984, Fay and Edds-Walton 1997a, Lu et al. 1996) but also to investigate frequency tuning in primary afferents (e.g. Fay and Edds-Walton 1997b, Lu et al. 2003, 2004, Meyer et al. 2004). The entire shaker system was positioned on a Micro-G pneumatic vibration isolation table.

Signals to the front-back shaker pair, side-to-side shaker pair, and the vertical shaker were digitally synthesized sinusoids (500 ms long) with $20 \mathrm{~ms}$ rise and fall times repeated eight times. Sinusoids were independently read out of the three channels of a 16 bit digital-to-analog converter (Tucker Davis Technologies, (TDT) DA) at a $10 \mathrm{kHz}$ sampling rate. Each signal was low-pass filtered at $2 \mathrm{kHz}$, controlled in amplitude by a TDT PA4 programmable attenuator, and amplified by a Techron power amplifier (Model 5507). The amplified signals were further attenuated by 32 $\mathrm{dB}$ using a resistor network to improve signal-to-noise ratio at the shaker inputs.

Three accelerometers (Piezotronics, PCB model 022A10, Flexcel) positioned on the vertical, side-to-side, and front-back axes were used to calibrate cylinder movement (at least once for all directional stimuli used in each experiment) before and after the fish was positioned in the stimulus dish. The accelerometer-output was monitored and used to make adjustments to the phases and amplitudes of the signals to create sinusoidal, linear movements along defined axes in the horizontal and vertical planes by use of a calibration program (Fay 1984).

The directional stimuli were presented at $100 \mathrm{~Hz}$ in six axes in the horizontal plane $\left(90^{\circ}, 60^{\circ}, 30^{\circ}, 0^{\circ}, 330^{\circ}\right.$, and $\left.300^{\circ}\right)$ and six axes in the vertical plane $\left(0^{\circ}, 30^{\circ}\right.$, 
$60^{\circ}, 90^{\circ}, 120^{\circ}$, and $\left.150^{\circ}\right)$. Once it was determined that most afferents innervating the saccule or lagena in A. fulvescens responded best to vertical stimulation at or near $90^{\circ}$ elevation, all stimuli testing the frequency response were set at $90^{\circ}$ vertical. Testfrequencies ranged between 50 and $1000 \mathrm{~Hz}$ for the first stimulus protocol. Once it was determined that the frequency response of afferents did not exceed $700 \mathrm{~Hz}$, the frequency range was reduced to 50 to $700 \mathrm{~Hz}(50,100,141,185,244,303,409,714)$. For isolevel frequency response functions, this stimulus protocol was repeated for several stimulus levels within the unit's dynamic range.

\section{Extracellular recording and data acquisition}

Extracellular recordings were made using $2 \mathrm{M} \mathrm{NaCl}$-filled glass pipettes with resistances between 20 and $50 \mathrm{M} \Omega$. These fine tip resistances were achieved using a micropipette puller (Sutter Instrument Co, P97). The micropipette was positioned on the nerve under visual control, and then advanced through with a remote hydraulic micro drive. The tip of the reference electrode (a silver silver-chloride wire of $1 \mathrm{~mm}$ diameter) was placed in the fluid of the brain cavity. Neural signals were amplified using a DAM 80 (World Precision Instruments) and band pass filtered between 300 to $3000 \mathrm{~Hz}$. A search stimulus $(100 \mathrm{~Hz})$ with constantly changing directional stimulation was used to maximize the chance of detecting a unit.

Response-level functions were determined for stimuli at $90^{\circ}$ elevation (updown), since this was the best axis for most afferents. Stimulus level was expressed as $\mathrm{dB}$ re $1 \mathrm{~nm}$ displacement rms throughout this study.

Spikes were discriminated using a single voltage criterion, and spike times were recorded with a $0.1 \mathrm{~ms}$ sampling period. From the spike times recorded during 
stimulation, period histograms (PHs), peristimulus time histograms (PSTHs), and inter spike interval histograms (ISIHs) were formed and the coefficient of synchronization $(\mathrm{R})$ was calculated to measure the degree of phase locking (Goldberg and Brown 1969). In order to minimize misinterpretations of $R$ when having a smaller number of spikes (Ns), the Rayleigh statistic $\mathrm{Z}=\mathrm{R}^{2} \mathrm{~N}_{\mathrm{s}}$ was applied to quantify phase locked responses (Batschelet 1981, Fay 1984). The $\mathrm{Z}$ value represented the response magnitude for afferents and for response level functions, the $\mathrm{Z}$ value was computed and plotted as a function of stimulus level.

Tuning curves and contour plots were created from response level functions (obtained for isolevel frequency response functions) by linear interpolation between levels.

\section{Results}

One hundred and seventy single units from 21 animals were recorded and analyzed. All of these units responded to sound with an increased spike rate and by phase-locking to the stimulus. About 90 units encountered during recordings did not respond to the stimulus and were not used for analysis. Responses were analyzed with respect to degree of phase locking, best frequency $(\mathrm{BF}$, determined from isolevel frequency response functions), characteristic frequency $(\mathrm{CF}$, determined from tuning curves), bandwidth (BW), rate-level functions, background rate and pattern, and temporal response pattern.

Recordings were made from the right posterior ramus of the eighth nerve innervating two otolithic end organs, the saccule and lagena, and the macula neglecta 
and posterior crista. It was not possible to access the nerve branches that innervated the saccule or lagena individually without damaging those epithelia. The electrode was always positioned over the dorsal surface of the right posterior ramus. While advancing the electrode from dorsal to ventral through the nerve branch, non-auditory units (units that did not phase-lock to the search stimulus) were found more dorsally, whereas auditory units were found more ventrally.

\section{Frequency selectivity of responses}

\section{Isolevel frequency response function}

All units that responded had a maximum peak in their isolevel frequency response function at one frequency, called BF. Units differed in their specific BF, in the sharpness of tuning around $\mathrm{BF}$, and in the shape of the isolevel frequency function. Some units (19\%; 33 out of a total of 170 units) had a second or third peak in their isolevel frequency response function (called "multi peak functions") versus $81 \%(137 / 170)$, with only one peak (“mono-peak functions").

Isolevel frequency functions were plotted using both spike rate and the Zvalue (which considers both spike rate and phase locking) as a measure of response strength (Fig. 2.1). However, it was decided to focus on the Z-value as a measure of response strength for the data-presentation in this paper since most units are spontaneously active and $99 \%$ of the units were strongly phase locked (with R values $>0.5$ at 10 to $15 \mathrm{~dB}$ above threshold). Figure 2.1 illustrates that the dynamic range 
and response profile of a spontaneous unit is reduced when choosing spike rate as compared to $\mathrm{Z}$ for the same range of levels. The response function in Figure .2.1 shows two peaks, one large peak at $100 \mathrm{~Hz}$ and a smaller one at $300 \mathrm{~Hz}$ at all levels. This response profile becomes less apparent when plotting spike rate as a function of frequency.

Isolevel frequency functions using spike rate as a measure of response strength, revealed that single tone suppression (Fay 1990) occurred in some units at the highest frequencies and intensities (e.g. Fig. 2.1 left). For unit pp9 spike rate decreased below the rate of background firing at 15 and $20 \mathrm{~dB}$ (re $1 \mathrm{~nm}$ displacement rms) at 400 and $700 \mathrm{~Hz}$. Generally, most afferents were spontaneously active, which means that they fired spikes in the absence of an intentional stimulus.

\section{Tuning curves}

Tuning curves were created from isolevel frequency functions for a subset of 65 units, sampled at levels within the unit's dynamic range and near the threshold of a unit. Tuning curves give insight into the lowest signal levels needed to evoke a response at a particular frequency. The threshold criterion used for the tuning curves was a $\mathrm{Z}$-value of 50 , which is a very conservative measure because at a $\mathrm{Z}$ of 50 there is only a very low probability that the phase locking of a unit is a random event $(\mathrm{p}=$ $0.000000001)$.

From tuning curves, the characteristic frequency $(\mathrm{CF})$, or the frequency that evoked a response at the lowest stimulus level, was determined. Usually, the CF 
corresponded to the BF of the same unit. Most tuning curves were V-shaped except for units with multi-peak functions.

Examples of mono-peak functions (representing the majority of all frequency response functions in A. fulvescens)

Most afferents $(81 \%, 137 / 170)$ had isolevel frequency functions with only one major peak. Figure 2.2 illustrates the isolevel frequency function and the corresponding tuning curve (plotted as a tuning-contour plot) for four different units. The black outline in the contour plot defines the unit's actual tuning curve (threshold criterion of $Z=50)$. Ff1 1 had an isolevel frequency response function with one single peak at $141 \mathrm{~Hz}$ at all levels tested other than the highest level where the BF shifts to $185 \mathrm{~Hz}$. An increase of BF at the highest level could be found in 4\% (6/137) of units with mono-peak functions. For some units, at higher stimulus levels the highest peak of the response function shifted towards a lower frequency (7\% or 9/137). Ff11 is broadly tuned, its tuning curve is V-shaped and looked band-pass in its filtering. Compared to ff11, unit qq5 was narrowly tuned and had a BF of $100 \mathrm{~Hz}$. It also had a V-shaped tuning curve and band-pass characteristics. Unit ss11 became more broadly tuned at higher signal levels, and showed a sudden increase of broadness at the highest level tested. With increase in level, the BW increased asymmetrically and the unit became especially more responsive to higher frequencies (seen in $4 \%$ of all units). Ss11 had a best frequency of $100 \mathrm{~Hz}$.

Units either had a symmetric V shape (e.g. ff11 and qq5) or had a slightly sharper cutoff towards lower frequencies than toward higher frequencies $(10 \%$; 
14/137) or vice versa $(10 \% ; 14 / 137)$. An example is unit tt3, which had a BF at 100 $\mathrm{Hz}$ and its narrowly tuned contour plot indicates a slightly sharper cutoff towards higher frequencies than towards lower frequencies.

\section{Multi-peak functions}

Some units (19\%, 33 out of a total of 170 units), had isolevel frequency response functions with more than one peak (e.g., Fig. 2.1). The profile of the isolevel-frequency function of most of these multi-peak units (18/33) stayed consistent across all levels tested. For some units, the second or third peak occurred only at the highest level tested (7/33), while others had multi-peak profiles at lower levels but lost the additional peaks at higher levels (8/33). These multi-peak-units were found in 12 different animals and were always found together with the majority of afferents having mono-peak response profiles.

Figure 2.3 shows an example of a unit (tt7) that had a response level function with three peaks, occurring at 100 (largest peak), 185, and $303 \mathrm{~Hz}$, and this profile remained consistent across all signal levels. The temporal pattern of the spontaneous activity shows distinct peaks, which occurred at 7, 17, 25, 33, $42 \mathrm{~ms}$ (Fig. $2.3 \mathrm{~B}$ ).

The response level function for unit tt7, as well as the PSTH, ISIH, and PH (Fig. $2.3 \mathrm{C}$ ) obtained at $100 \mathrm{~Hz}$ within the unit's dynamic range, show that this was most likely a single-unit and that the multiple peaks were not caused by a second afferent from a multi-unit recording. 
Best frequency distribution and sharpness of tuning curves

The majority of afferents (59\%; 81 out of 137 afferents with mono-peak functions) had BFs at $100 \mathrm{~Hz}$, while $31 \%(43 / 137)$ had their BF at $141 \mathrm{~Hz}$. BFs of only $10 \%(13 / 137)$ of the afferents exceeded $141 \mathrm{~Hz}$ with one afferent having a $\mathrm{BF}$ at $303 \mathrm{~Hz}$ (the maximum BF found, Fig. 2.4 A).

To assess the sharpness of a frequency response, $\mathrm{Q}_{10 \mathrm{~dB}}$ (dividing the CF by the bandwidth, $\mathrm{BW}$, at $10 \mathrm{~dB}$ above threshold $)$ was determined for tuning curves $(\mathrm{n}=$ 36). $\mathrm{Q}_{10 \mathrm{~dB}}$ was chosen, since this measure of bandwidth is widely used in the vertebrate literature. The absolute values of bandwidths (Fig. 2.4 B, left) determined at $10 \mathrm{~dB}$ above threshold at $\mathrm{CF}$ are presented together with the $\mathrm{Q}_{10 \mathrm{~dB}}$ values (Fig. 2.4 $\mathrm{B}$, right). $\mathrm{BW}$ is more intuitive for describing the sharpness of a filter while $\mathrm{Q}_{10 \mathrm{~dB}}$ is more comparable to data in other vertebrates. Units differed widely in sharpness and the distributions for BW and $\mathrm{Q}_{10 \mathrm{~dB}}$ are both Poisson-like.

Plotting the relationship between BW and CF (Fig. 2.5, left) revealed that the two parameters were significantly correlated (the same was found plotting $\mathrm{Q}_{10 \mathrm{~dB}}$ with CF however the correlation was not as strong; Fig. 2.5, right). With increase in CF, the bandwidths of tuning curves increased, thus the sharpness of the units decreased (BW with CF: $\mathrm{R}=+0.73, \mathrm{Q}_{10 \mathrm{~dB}}$ with $\mathrm{CF}: \mathrm{R}=-0.34, \mathrm{n}=36$, two-tailed; see also table $1)$.

Summary of correlations between parameters

Table 2.1 summarizes the results regarding correlations between parameters. Correlation values between bandwidth and CF were highest (as described above). 
There seemed to be a correlation between bandwidth and spontaneous rate indicating that the bandwidth of units with higher spontaneous rates tended to be smaller (however there was no correlation between $\mathrm{Q}_{10 \mathrm{~dB}}$ and spontaneous rate). Correlation values tested for Q-values instead of bandwidths were generally lower. There was no correlation between BW (or Q-values) with threshold but there was a correlation between spontaneous rates and BFs. Units with higher BFs tended to have a lower spontaneous rate.

\section{Displacement, velocity, and acceleration tuning curves}

The tuning curves (obtained at the stimulus level in $\mathrm{dB}$ re $1 \mathrm{~nm}$ displacement) of several units with different shapes, bandwidths or CFs were converted into $\mathrm{dB}$ re $1 \mathrm{~nm} / \mathrm{s}$ velocity and $\mathrm{dB}$ re $1 \mathrm{~nm} / \mathrm{s}^{2}$ acceleration (Fig. 2.6). The outcome of this conversion was that no matter which shape the tuning curve had (even W-shaped,

Fig. 2.6 B, unit pp9), when converted to acceleration, all units became low-pass filters with corner frequencies that corresponded to the BF. The sensitivity to frequencies higher than the corner frequency decreased very rapidly.

\section{Response level functions}

Response (Z) versus level functions of a given afferent to sinusoidal stimulation at $100 \mathrm{~Hz}$ differed widely in threshold but all had similar shapes, which were monotonically increasing up to a certain saturation point (some examples, Fig. 2.7 A). Their slopes varied between 14 to $28 \mathrm{Z} / \mathrm{dB}$ and their dynamic ranges were between 9 to $24 \mathrm{~dB}$. 
When considering the variations of response level functions of one unit with frequency (Fig. 2.7 B, plotted for 102 units), it becomes apparent that the slope is steepest at $\mathrm{BF}$ and becomes flatter with increasing or decreasing frequencies relative to $\mathrm{BF}$, as can also be inferred from isolevel frequency functions obtained at different levels shown in Figure 2.7. This was observed in all 102 units independent of monopeak or multi-peak profile.

\section{Pattern of background firing}

Generally, background activity (or spontaneous activity) was defined as firing of spikes in the absence of any intentional stimulus. The background pattern was investigated for a total of 82 afferents (randomly selected), which showed clear frequency (and directional) responses by plotting ISIHs for each individual afferent. Figure 2.8 A shows an example, in which the intervals between spikes of this unit (pp10) were Poisson-like distributed. Most afferents 63\% (52/82) had spontaneous activity patterns resembling the one shown in Figure 2.8 A. For other afferents (37\%, 30/82), the distribution of spikes was Gaussian-like as shown in the example of Figure $2.8 \mathrm{~B}$.

Units having a bursting spontaneous activity pattern were also found, with 11 units showing one distinct peak at a smaller interval (double spike bursts) and a larger continuous distribution of intervals between spikes that was either Gaussian- or Poisson-like. Nine units had multiple distinct peaks in their ISIH (see above, example of multi-peak unit, Fig. 2.3 B). 


\section{Temporal response pattern, interval distribution, and phase locking in responding afferents}

The temporal response pattern (PSTH), inter spike interval distribution (ISIH), and the degree of phase locking (PH) to $100 \mathrm{~Hz}$ stimulation were investigated for 53 units from 17 animals. Most units $(85 \%, 45 / 53)$ fired continuously and in a highly phase-locked manner to the stimulus ("tonic firing pattern"; see example Fig. 2.9 A, unit uu8). Uu8 showed strong phase locking (as indicated by a distinct peak in its $\mathrm{PH}$ ). At higher levels, the degree of phase locking became even stronger (slimmer peak) and the phase angle decreased slightly with level. At the highest levels the ISIHs showed only one peak indicating that the unit did not skip any cycles of the stimulus waveform.

Fifteen percent of the units $(8 / 53)$ showed a phasic tonic response pattern, which means that the afferents fired more spikes during the onset of the stimulus. The example shown in Figure 2.9 B (pp7) showed a phasic tonic response pattern that appeared consistently across all levels (even near threshold at 14dB) but became stronger with increasing stimulus intensity. This unit was also highly phase-locked (see distinct peaks in its ISIH related to the period of the stimulus waveform and single peak in its $\mathrm{PH})$. The phase angle decreased only slightly with increasing intensity.

Some units firing in a tonic or phasic manner showed bursting $(15 \%, 8 / 53)$. These bursts were usually double spikes occurring at a very short interval even at low levels (Fig. 2.9 C) near threshold. More bursting occurred as stimulus level increased. The ISIH shows one sharp peak smaller than $5 \mathrm{~ms}$ (half the period of the stimulus waveform) and one or more other peaks that indicate phase coupling (occurring at or 
near the integer multiples of the stimulus period). The PHs show two peaks at higher levels that become more and more distinct with increasing level, thus both spikes were phase-locked and occurred at different phase angles but they were not $180^{\circ}$ apart from each other.

\section{Discussion}

The responses of eighth nerve afferents to stimuli varying in frequency and intensity in A. fulvescens showed strong phase coupling and tuning curves centered on a few different frequencies. Most afferents showed background activity, and for some afferents the background rate decreased at higher frequencies (than BF; single tone suppression). The tuning curves varied quite a lot in sharpness and shape, and responses occurred at a wide range of intensities. In the following section data will be compared to teleost fishes, as well as to other vertebrates.

\section{Spike rate versus $Z$ based observations}

Frequency response functions were plotted twice, as spike rate versus frequency and as $\mathrm{Z}$ versus frequency. Generally, the response functions had similar shapes and BFs. The threshold for spontaneously active units was defined as the spontaneous rate plus one standard deviation of the background rate and was used to compare the Z-based threshold with a spike rate based threshold. The comparison revealed that thresholds for spontaneously active afferents were elevated by up to 10 $\mathrm{dB}$ (re 1nm displacement) when using spike rate as a measure of response strength, which is comparable to findings in other vertebrates (e.g. birds, Gleich and Manley 
2000). Thus, auditory nerve fibers tended to rearrange their spontaneous discharge in the time domain to follow the stimulus cycles without raising the discharge rate (Fay and Coombs 1983).

Isolevel frequency functions using spike rate as a measure of response strength, showed that single tone suppression occurred in some units at the highest frequencies and intensities (Fig. 2.1 left). Single tone suppression has been observed in all vertebrate species (reviewed in Gleich and Manley 2000, and for fish: Fay 1990). The firm coupling between the tectorial membrane and the stereovillar bundle may play a role in producing single-tone suppression in nonmammalian species with a tectorium (Gleich and Manley 2000). The origin for this phenomenon in fish is unknown.

\section{Frequency tuning in afferents}

The shape of the tuning curves of most units resembled a band-pass filter but some appeared more asymmetric, having either a sharper cut off towards lower or higher frequencies (e.g. compare ss 11 with tt3, Fig. 2.2). These variations in shape and bandwidths resembled those seen in teleosts, for example in saccular afferents of P. notatus (Weeg et al. 2002) and in lagenar afferents of C. auratus (Meyer et al. 2004). A change of BF with increasing level (generally an increase of BF) has also been described for P. notatus and C. auratus, and for utricular afferents in D. latifrons (Lu et al. 2004). A decrease of BF with level as seen in A. fulvescens, has been found in C.auratus (only for high-frequency saccular afferents; Lu and Fay 1993) and in mammalian afferents (reviewed in Ruggero 1992). 


\section{Multi-peak functions}

While the tuning curves of most units were V-shaped (as it is typical for auditory systems in other vertebrates), some units (19\%) showed W-shaped tuning curves and isolevel frequency functions with more than one peak (max. three peaks). A few frequency response functions with multiple peaks have also been found in saccular afferents of $P$. notatus (Weeg et al. 2002), lagenar afferents of $C$. auratus (Meyer et al. 2004), and in utricular afferents of D. latifrons (Lu et al. 2004). Units with multi-peak response functions were also found in the medulla and midbrain of O. tau (Edds-Walton and Fay 2003).

One unit with a multi-peak response profile is shown in Figure 2.3 (tt7). The ISIH of the spontaneous activity, response level functions, and response pattern were investigated for this unit (and for others of this type). These data did not look different from units with mono-peak functions indicating that the multiple peaks most likely were not a result of recordings from multiple afferents at the same time.

For unit $\mathrm{tt} 7$, the main intervals of the ISIH did not occur at the periods (or integer multiples of the period) of the frequency it maximally responded to $(100 \mathrm{~Hz})$. Thus, it is unclear whether one can consider these intervals as "preferred intervals" found in other vertebrates (Manley 1990). For instance, Eatock and Manley (1981) found that low-frequency units in Gecko gecko (tokay gecko) had intervals (of their

spontaneous ISIH) that occurred at the period or integer multiples of the period of the $\mathrm{CF}$ of the unit. Preferred intervals were considered to reflect electrical tuning in nonmammalian hair cells (at low CFs; Manley 2000). 
However, the frequency resolution of the test-stimuli in the study on $A$. fulvesens was such that it left ambiguity in terms of the exact $\mathrm{BF}$ or $\mathrm{CF}$ of a unit. For example a peak at $100 \mathrm{~Hz}$ could reflect any BF between 75 and $120 \mathrm{~Hz}$, since stimulus-frequencies were set at 50,100,141 Hz and not at frequencies in between. In future experiments, it would be useful to stimulate afferents at smaller frequency intervals to determine if ISIH-peaks of spontaneous activity are within the period (and integer multiples) of the CF or not.

The frequency peaks of the response profile of unit tt7 occurred at 100, 185, and $303 \mathrm{~Hz}$. These peaks were harmonically related (when considering $185 \mathrm{~Hz}$ being close enough to $200 \mathrm{~Hz}$ ) and there was the possibility that the peaks were caused by subharmonics in the stimulus. However, units with multi-peak functions were found in 12 different experiments and always occurred together with the majority of afferents having mono-peak response profiles. During calibration (sometimes repeated at a later stage of the experiment), no subharmonics or other stimulus artifacts were detected.

In summary, units with multiple peaks in their isolevel frequency functions or tuning curves are unusual among vertebrates, but do occur in teleosts. One such afferent may innervate hair cells with multiple resonances or different BFs. However, the input from hair cells with different BFs is rather unlikely since these individual hair cells would have to be very narrowly tuned. The directional response profiles (general discussion for A. fulvescens see paper on directional tuning) of those units looked like a cosine function indicating that hair cells innervated by any single afferent seemed to be consistent in their orientation. 


\section{Best frequency range and hearing}

The distribution of BFs (or CFs for tuning curves) for 137 afferents (with mono-peak response profiles) was Poisson-like. The majority of afferents had BFs at $100 \mathrm{~Hz}(59 \%)$ or $141 \mathrm{~Hz}(31 \%)$ and only one afferent had its BF at $303 \mathrm{~Hz}$. When stimulated with the same stimulation method used in this study, CFs in saccular, lagenar, and utricular afferents in D. latifrons clustered between $\leq 50$ and $125 \mathrm{~Hz}(\mathrm{Lu}$ et al. 2003, 2004), in P. notatus between $\leq 50$ and $200 \mathrm{~Hz}$ (Weeg et al. 2002), and in O. tau between $\leq 50$ and $250 \mathrm{~Hz}$ (Fay and Edds-Walton 1997b).

A. fulvescens had similar BFs to the teleost species mentioned above which have been classified as hearing non-specialists except no afferents were found that indicated a strong response to frequencies smaller than $100 \mathrm{~Hz}$ in A.fulvescens. In contrast, strong responses were found for example in utricular afferents of $D$. latifrons, saccular afferents in P. notatus, and for lagenar afferents in C. auratus (Lu et al. 2004, Weeg et al. 2002, Meyer et al. 2004).

While BFs in A. fulvescens only ranged between 100 and $300 \mathrm{~Hz}$, some units responded to higher frequencies only at the onset of the stimulus (through a highly phasic response). In addition, the response of some of the units, even though they had their BFs at lower frequencies, extended to frequencies up to $700 \mathrm{~Hz}$. Thus, the total frequency range to which saccular/lagenar afferents in A. fulvescens responded was between 50 and $700 \mathrm{~Hz}$.

While the stimulus used in this study was particle motion, previous recordings of auditory evoked potentials (AEP) used a loudspeaker submerged in water displaying pressure as well as particle motion of sound (Meyer et al. 2002). 
Responses in A. fulvescens occurred up to $2000 \mathrm{~Hz}$. The lowest levels needed to evoke an AEP occurred between 100 and $300 \mathrm{~Hz}$, thus confirming the results found in this study using particle motion only. At frequencies higher than $300 \mathrm{~Hz}$, stimulus levels needed to evoke an AEP increased steeply. A comparable best frequency range for AEPs was also found by Lovell et al. (2005) with lowest thresholds (using sound pressure stimulation) occurring between 200 and $250 \mathrm{~Hz}$ in A. fulvescens. Responses to frequencies larger than $700 \mathrm{~Hz}$ in AEP-studies may be explained by a potential pressure based input via the swim bladder in A. fulvescens. While the bladder is located in some distance to the ear in A. fulvescens (personal observation), it may still be possible that some of the sound pressure based energy that causes the swim bladder to pulsate in the sound field is directed to the ear in the form of (internal) particle motion. Cardiac conditioning experiments in Anguilla Anguilla (European eel, Jerko et al. 1989) revealed that the swim bladder is located in great distance to the ear (about $10 \mathrm{~cm}$ in a $50 \mathrm{~cm}$ long animal), yet the pressure based input from the swim bladder increased the sensitivity to higher frequencies in this species. Since special connections of the swim bladder to the ear were not found in A. Anguilla, authors assumed that bladder movements may be transmitted through ordinary body tissue and that the transmission channel for particle motion between swim bladder and ear is more efficient that just water.

Generally, in AEP- studies, a loudspeaker (creating both pressure and particle motion components) for stimulation and a pressure-based calibration was used. Therefore, a comparison to those studies can only be made in terms of frequency range but not absolute thresholds. The current study based on single unit recordings 
instead, gives insight into absolute thresholds of the ear with regard to particle motion and frequency range (and therefore a better idea of an audiogram for the auditory/ vestibular periphery), as well as into the coding strategies of the auditory (or vestibular) periphery.

Vocalizations are not known in A. fulvescens. One study recorded vocalizations by adult Scaphyrinchus albus (pallid sturgeon) and Scaphyrinchus platorynchus (shovelnose sturgeon) in holding tanks. The dominant frequencies of sounds produced by these species ranged between 90 to $4,200 \mathrm{~Hz}$ (Johnston and Phillips 2003).

While fishes use sounds in behaviors including aggression, defense, courtship, and mating (reviewed in Popper et al. 2002), they are likely to use sound also for gaining information about the environment around them (the auditory scene) to detect prey and escape from predators, as well as to identify locations (Sand and Karlsen 2000, Popper and Fay 1997, Fay and Popper 2000). Most species of the family Acipenseridae are anadromous, which means that they spend most of their lives at sea but ultimately return to their spawning grounds in rivers, sometimes migrating long distances upstream. They are known to have strong homing capabilities (Kynard 1997), and sound could potentially be one of the cues to a particular location that these species use. A. fulvescens can detect particle motion of sound between 50 and $700 \mathrm{~Hz}$, a frequency range that lies within the range for ambient sounds (Scheifele and Darre 2005). A. fulvescens spawns in shallow waters. Since higher frequencies propagate better in shallow waters (however within a shorter range, Rogers and Cox 1988), a detection of higher frequencies (as found in AEP studies for frequencies up 
to $2000 \mathrm{~Hz}$ ) may be useful during spawning, while lower frequencies (up to $700 \mathrm{~Hz}$ ) may be more important during the time animals live in deeper waters (lakes or in the sea for some sturgeon species).

Sharpness in primary afferents innervating saccule or lagena in A. fulvescens

$\mathrm{Q}_{10 \mathrm{~dB}}-\mathrm{values}$ determined for 36 units in A. fulvescens ranged between 0.6 and 1.8 (mean: $1.06 \pm 0.42 \mathrm{SD}$ ). The higher $\mathrm{Q}_{10 \mathrm{~dB}}$, as sharper the unit is tuned. In the chinchilla auditory nerve $\mathrm{Q}_{10 \mathrm{~dB}}$ varied between one at $\mathrm{CFs}$ around $150 \mathrm{~Hz}$ to seven at CFs near $16 \mathrm{kHz}$ (Dallos and Harris 1978). In the auditory periphery of Gekko gecko (Tokay gecko; Eatock et al. 1981), the average $\mathrm{Q}_{10 \mathrm{~dB}}$ increased from two to three at low CFs (200 to $400 \mathrm{~Hz}$ ) to nearly eight at high CFs (2 to $4 \mathrm{kHz}$ ). In the rostral amphibian papilla (AP) of frogs, afferent fibers with low CFs $(100-400 \mathrm{~Hz})$ had $\mathrm{Q}_{10 \mathrm{~dB}}-$ values as high as five. Afferent fibers with high CFs from either caudal AP regions (CF-range: 400 to $1.4 \mathrm{kHz}$ ) or the basillar papilla (BP; CF-range: > $1 \mathrm{kHz}$ ) were more broadly tuned and rarely exceeded $\mathrm{Q}_{10 \mathrm{~dB}}$ values of two. Thus, in mammals, reptiles, and birds, sharpness increased with increasing CF (mammals: Kiang et al. 1965, reptiles: Manley 1990, birds: Gleich and Manley 2000). In frogs (e.g., Rana pipiens, the Northern leopard frog), the sharpness of tuning decreased with CF (Ronken 1991, reviewed in Simmons et al. 2007).

In summary, the sharpness of the tuning curves in A. fulvescens was comparable to the low frequency afferents of mammals, reptiles, and birds, and to the afferents innervating the caudal AP regions or the BP in frogs. As in frogs, there also 
seemed to be a decrease of sharpness of tuning with increasing CF (Fig. 2.5) in A. fulvescens. This correlation might add an interesting point for the discussion on the mechanisms of tuning in fish (and maybe frogs) contrasting it to the mechanisms from reptiles, birds, and mammals (showing the opposite trend).

\section{Frequency coding}

Tuning curves in A. fulvescens varied in sensitivity (threshold at CF), shape, sharpness, and CF. While there is not as much variation in $\mathrm{CF}$ (or BF for isolevel frequency functions, Fig. 2.5 A), the diversity in shape and sensitivity may help to create a specific signature or pattern across a population of afferents being stimulated by a given sound of a certain frequency and intensity. A pattern representing stimulus features such as frequency and intensity could be encoded centrally. In addition, the activation of only a few afferents differing in CF may be enough to encode a particular frequency by central neurons, for example the dominant frequency of vocalizations. Thus, such a code based on spike rate relying on filters centered around a few different frequencies but very variable in shape may not require tonotopicity and may be the most primitive code for spectral analysis in vertebrates.

Revcor experiments (using the reverse correlation method) testing frequency responses in C. auratus and $O$. tau support the view that fish differ from other vertebrate species in having a small number of differently tuned frequency channels compared with a filter bank found in amphibians, reptiles, birds, and mammals (Fay 1999).

There was no indication of a systematic decrease or increase of BFs related to the recording location on the nerve of A. fulvescens. While labeling studies (filling a 
fiber with a neural tracer and identifying the termination site of the fiber at the epithelium) and sampling at finer frequency increments could be undertaken to systematically investigate the possibility of tonotopic organization in A. fulvescens, past studies in teleosts species (especially hearing non-specialists) showed that the ears did not have any tonotopic organization. A very crude organization was found in C. auratus (reviewed in Popper and Fay 1999) in that high-CF afferents originated primarily from the rostral region of the saccule, while low-CF afferents innervated the caudal region (Furukawa and Ishii 1967, Sento and Furukawa 1987). However, hair cells with similar oscillation-frequencies (up to $220 \mathrm{~Hz}$ ) were found on the rostral as well as caudal end of the saccule in C. auratus (Sugihara and Furukawa 1989).

At the peripheral level of the auditory system, frequency information was also preserved by phase locking in A. fulvescens within its entire frequency range of hearing (temporal code). In all vertebrate systems, a temporal code of neural information encodes frequencies by primary afferents that are highly phase-locked to acoustic waveforms up to 4 to $5 \mathrm{kHz}$. Thus, lower frequencies can be encoded by a temporal code and by a spike rate code in vertebrates. One function of a temporal code could also be to increase the dynamic range of a spontaneous unit since thresholds are lower when considering phase locking versus spike rate in a spontaneous unit (as discussed above).

For frequencies above 4 to $5 \mathrm{kHz}$ (relevant for reptiles, birds, mammals, and ultrasound-sensitive fish), the only information about the frequency spectrum of sounds that is available to central auditory processing units is that contained in the 
distribution of discharge rate across the auditory nerve fiber array (reviewed in Irvine 1992, Fay 1999).

In summary, this study shows that in an ancestral vertebrate such as $A$.

fulvescens, frequency information is encoded by both temporal and rate

characteristics of spike trains. Most fish species have hearing ranges below $4 \mathrm{kHz}$.

Thus, a temporal code is sufficient to encode frequencies at this range. However, some teleost fish can detect ultrasound (e.g., Alosa sapidissima, American shad;

Mann et al. 1997, Mann et al. 2001). It is possible that the existence of rate coding in ancestral fish, such as A. fulvescens, "paved the way" for rate coding of higher frequencies (ultrasound) in clupeiformes, assuming that results found in A. fulvescens represent the ancestral condition for frequency coding in bony fish (for more discussion see summary and evolutionary considerations below).

\section{Displacement, velocity, and acceleration tuning curves}

When the tuning curves are plotted using acceleration rather than displacement as the stimulus parameter they appear nearly flat at the lower frequencies. This tuning curve shape is consistent with the idea that the otolith organ is a damped simple harmonic oscillator driven by an external input and the output of the organ is proportional to the displacement of the haircell bundle (de Vries 1950). This arrangement acts as an accelerometer. The output of an ideal accelerometer in response to sinusoidal acceleration depends on frequency (Kalmijn 1988, 1989). At low frequencies, the output displacement depends only on the magnitude $(A)$ of acceleration, thus the system acts like a pure accelerometer (stiffness controlled). At 
intermediate frequencies, the output displacement is proportional to $\frac{A}{\omega}$ (where $\omega$ is the input frequency), thus the system acts like a velocity detector (since this corresponds to the process of integration going from acceleration to velocity). The system at this point is predominantly controlled by viscous drag (acting against the moving mass). At higher frequencies the output displacement instead is proportional to $\frac{A}{\omega^{2}}$ (corresponding to the process of integration going from acceleration to displacement), thus the system becomes a displacement detector (mainly controlled by the inertia of the moving mass).

Thus, plotting intensity as $\mathrm{dB} \mathrm{re} 1 \mathrm{~nm} / \mathrm{s}^{2}$ acceleration (instead of $\mathrm{dB}$ re $1 \mathrm{~nm}$ displacement) for tuning curves may be the better way to show that otolith organs are indeed functioning as accelerometers, as suggested by de Vries (1950), Kalmijn (1988), and Sand and Karlsen (2000). The corner frequency (formerly BF) marks the frequency range up to which the system is most sensitive to stimulus acceleration.

\section{The role of the saccule and the lagena in hearing}

Frequency responses to whole-body acceleration were determined for all three otolithic organ afferents in D. latifrons (Buchser et al. 2003, Lu et al. 2003, 2004). Results showed that saccular, lagenar, and utricular afferents had very similar CFs and that units showed tuning curves appearing like a band pass typical for auditory fibers. Thus, a similarity in response characteristics (with respect to $\mathrm{CF}$ and tuning curve shape) between saccular, lagenar, and utricular afferents to particle motion 
suggested that all three otolithic organs might play a role in hearing (probably in addition to vestibular function).

The saccule and lagena in A. fulvescens are likely to be involved in hearing since tuning curves appeared more like a band pass and all physiological characteristics showed strong resemblance to data on the saccule in teleost fishes (Fay 1984, Fay and Edds-Walton 1997, Weeg et al. 2002, Buchser et al. 2003) which is considered to be involved in hearing. Possibly, otolith organs in fishes have a dual function, both vestibular and auditory (Platt 1989, reviewed in Popper et al. 2002).

There was no dichotomy in the data for A. fulvescens, suggesting a functional separation between the saccule and lagena. However, labeling studies of individual afferents and lesion studies together with behavioral studies might assess more clearly to what extend individual otolith organs are involved in auditory and vestibular function. It may also be useful to investigate the response properties of utricular afferents in A. fulvescens to assess the potential role of the utricle for auditory (and vestibular) function.

\section{Response level functions and coding of level}

Response level functions in A. fulvescens, were monotonically increasing with increasing stimulus level up to a saturation point. The dynamic ranges of response level functions were comparable to data from saccular afferents in $O$. tau (Fay and Edds-Walton 1997a) and lagenar and utricular afferents in D. latifrons (Lu et al. 2003, 2004). Generally, the dynamic ranges of individual afferents seemed to be smaller in fish than for some auditory afferents in other vertebrates (e.g. max. $40 \mathrm{~dB}$ re SPL mammals, Kiang et al. 1965; max. 40 dB re SPL in reptiles, Eatock and Weiss 
1986; max. 50 dB re SPL in frogs, Simmons et al. 2007). However, the wide variation in thresholds for saccular and lagenar afferents in A. fulvescens, also seen in teleost species (C. auratus: Fay 1984, Meyer et al. 2004, O.tau: Fay et al. 1997), may compensate for the limited dynamic range of individual fibers. A higher range of intensities can be encoded by a population of afferents in fish having different thresholds at a wide range of levels.

Generally in vertebrates, the range of stimulus levels over which the response strength of individual response level functions changes is much smaller than the behavioral range of intensities that can be detected; the "dynamic range problem" (Kiang et al. 1965, reviewed in Irvine 1992). It was proposed that intensity discrimination at high intensities is based on a mall proportion of auditory nerve fibers that have elevated thresholds and an extended dynamic range (Liberman 1978), thus stressing a population code to solve this problem.

A sigmoidal, monotonic shape (as seen in all response level functions of $A$. fulvescens) as opposed to a non-monotonic function seen in some vertebrates (e.g. mammals, Kiang et al. 1965) decreases ambiguity and is thus optimal for coding intensities by a population of afferents. The lack of non-monotonic functions may be attributed to the finding that many afferents in A. fulvescens showed a tonic firing (when stimulated at $\mathrm{CF}$ ) even at the highest stimulus levels.

The dynamic range among afferents varied, however the Z-spread was similar among units. Units with a shorter dynamic range but a similar Z-spread would have higher sensitivities to changes in stimulus level. 
Figure 2.7 B shows that slopes of the response level functions of one unit (CF $=141 \mathrm{~Hz}$ ) decreased with frequencies lower and higher than CF in A. fulvescens. A temporal response pattern of units changing with frequency has not been investigated in A. fulvescens but the decreasing slopes at higher frequencies (than CF) might have been caused by a progressively more phasic nature of the responses as found for instance in lizards (Manley 1992). However, afferents firing increasingly with a phasic response pattern with increasing frequency can lead to non-monotonic response level functions, which were not found in A. fulvescens.

Whether slopes and dynamic ranges of response level functions varied in afferents with CFs other than $100 \mathrm{~Hz}$, remained to be investigated, since all response level data for sturgeon are obtained at $100 \mathrm{~Hz}$.

\section{Background activity pattern}

Inter spike interval histograms (ISIHs) obtained for the background activity of frequency selective units in sturgeon were either Poisson distributed (63\%, Fig. 2.8 A) or normally distributed (37\%, Fig. 2.8 B) in A. fulvescens. Some background activity patterns showed bursting which lead to a bimodal distribution of interspike intervals or to multiple peaks. Poisson-like distributions of spontaneous action potentials are common in mammals, birds and reptiles and they are generally attributed to a stochastic release of transmitter packets at the afferent synapse in mammals (Kiang et al. 1965, Gleich and Manley 1992). Most common in teleost saccular afferents were units showing a random firing or bursting firing (with bimodal distributions of inter spike intervals; reviewed in Popper and Fay 1999). 
Units with higher BFs tended to have a lower background rate in contrast to observations in domestic cats (domestic cat; Kiang et al. 1965) and frogs (Rana temporaria, grassfrog; Christensen-Daalsgard et al. 1998) (see Table 2.1). It remains unclear why units with higher BFs would fire at lower background rates. There were no clear correlations between background rate and sharpness (expressed as $Q_{10 \mathrm{~dB}}$ ) as found in cats (Kiang et al. 1965).

Most fibers showed background activity in A. fulvescens. Background activity can add to an increase of the dynamic range for coding since the background activity was sometimes suppressed, such as seen during single tone suppression of some afferents or during suppression after stimulus-offset.

\section{Temporal response pattern}

Most units in A. fulvescens showed a tonic response pattern over the entire duration of the stimulus presentation. The sustained pattern remained unchanged with increasing level (Fig. 2.9 A). This pattern was common in lagenar and utricular fibers of D. latifrons (Lu et al. 2003, 2004) classified as “entrained phase-locking”.

The phase of the waveform at which the peak occurred in the period histogram tended to decrease with stimulus level in A. fulvescens (Fig. 2.9 A, right). This has also been found in saccular afferents of $O$. tau and in saccular and lagenar afferents of $C$. auratus with either low or no spontaneous activity (Fay et al. 1996, Fay and Edds-Walton 1997a, Meyer et al. 2004). In afferents with very high background activity, spikes tended to occur at the same phase or time with respect to the stimulus waveform regardless of sound level within an afferent's dynamic range 
in A. fulvescens (for a detailed discussion refer to directional paper on A. fulvescens) as well as in C. auratus and $O$. tau.

Units with phasic tonic response patterns were also present (15\%; Fig. 2.9 B). Units with phasic tonic response pattern were generally as strongly phase coupled to the stimulus waveform as those with a tonic response pattern. Unit pp7 (Fig. 2.9 B) showed only a small decrease in phase angle with level as compared to unit uu8 having no spontaneous activity (Fig. 2.9 A) as discussed above. This unit however was spontaneously active whereas uu8 was not. A phasic tonic response pattern is very common in vertebrate auditory systems (Gleich and Manley 2000, Ruggero 1992). Background rates are often suppressed after stimulus presentation at higher levels in vertebrate auditory fibers but eventually return to pre-stimulus levels. This was also observed during experiments with A. fulvescens (not shown in Fig. 2.9) and demonstrated in saccular or lagenar afferents of Acipenser brevirostrum stimulated by a loudspeaker under water (Meyer et al. 2003).

A few afferents in A. fulvescens showed phase-locked bursting. Phase-locked bursters have also been shown in lagenar and utricular afferent of D. latifrons (Lu et al. 2003, 2004). In D. latifrons, lagenar afferents that showed no phase locking tended to have a phasic response pattern ( $\mathrm{Lu}$ et al. 2003). Phasic tonic response patterns were instead found in strongly and weakly phase-locking afferents in D. latifrons.

Double phase-lockers could not be found in A. fulvescens but have been described for utricular and lagenar afferents in D. latifrons and saccular afferents in C. auratus (Furukawa and Ishii 1967). Double phase-lockers in D. latifrons had period histograms with two peaks that were $180^{\circ}$ apart from each other. It is generally 
believed that those afferents innervate hair cells with opposing bundle orientations with one group of hair cells responding to the compression the other (opposing) group of hair cells responding to the rarefaction phase of sound (Furukawa and Ishii 1967). Thus, afferents in A. fulvescens may not innervate groups of hair cells with opposing orientations.

While the effect of frequency on temporal response patterns was not investigated, it was noticed during the experiments that some units fired at high frequencies (higher than $300 \mathrm{~Hz}$ ) in a purely phasic manner (just at the onset of the stimulus).

\section{Summary and evolutionary considerations}

This is the first study of the response properties of primary auditory afferents in a non-teleost bony fish focusing on a detailed physiological analysis of frequency and intensity coding and the first investigation on sensory coding in a non-teleost bony fish in general. In addition, the hearing range and sensitivity in a non-teleost bony fish was discussed placing it in a neuroethological context.

The hearing range for A. fulvescens was estimated from best frequencies and maximal tuning curve widths determined for saccular and lagenar afferents stimulated by particle motion. Afferents responded to a total frequency range between 50 and $700 \mathrm{~Hz}$ with best responses between 100 and $300 \mathrm{~Hz}$. The estimated total hearing range thus resembles the ones found in teleost hearing non-specialists. However, the results for A. fulvescens differed to teleosts in that no afferents were found that indicated a strong response to frequencies smaller than $100 \mathrm{~Hz}$ in comparison to 
teleost hearing non-specialist (using a similar stimulation method) and to the lagenar afferents in C. auratus (Meyer et al. 2004) The hearing range may enable $A$. fulvescens to use sounds for migration and other aspects of auditory scene analysis and to encode lower frequency vocalizations.

While the best frequencies and frequency range did not vary much between otolith organs in D. latifrons (the only species for which frequency data of all three otolith organs are known), it may still be useful to measure the response properties of utricular afferents in A. fulvescens to investigate the potential role of the utricle for auditory (and vestibular) function.

Towards an understanding of the origin of hearing

Recordings from primary afferents innervating the saccule and lagena in $A$. fulvescens showed many similarities to the findings described for the auditory (or vestibular) periphery in teleost fishes and to auditory systems in vertebrates. Among those findings were strong phase coupling, single tone suppression, band pass-like tuning curves, variation in shape and sharpness of tuning curves, a few frequency filters (as in teleosts), a very well suited system to encode level through population coding, variable response pattern (as in teleosts), and variable spontaneous activity pattern (as in teleosts and other vertebrates).

The similarities to teleosts are interesting considering that A. fulvescens belongs to a group of fishes that had their major radiation hundreds of million years ago and broke off from the teleost radiation approximately 250 million years ago. The similarities to other vertebrates (and teleosts) is also interesting when assuming as a 
first approximation that the coding strategies found in A. fulvescens may represent the ancestral condition for all vertebrates, at least as far back as the origin of the bony fishes. However, it should be noted that comparing more species of the group of nonteleost bony fish would be necessary to make a full assessment of ancestral versus more modern coding strategies (within the group of bony fishes), since A. fulvescens may have developed modern advanced characteristics during its own evolutionary course.

An alternative way to interpret the results found in A. fulvescens is that similar coding strategies for frequency and intensity evolved independently in distantly related species (convergent evolution). This would indicate the possibility that selective pressures lead to only a few coding strategies that a vertebrate auditory system can evolve. However, it seems likely that similiarities occurred because the auditory system in vertebrates derived from a common ancestor. The assumption is based on the notion that certain auditory/vestibular structures are considered homologous, such as the vertebrate hair cell (Coffin et al. 2004) and that the gross morphology of the inner ears is very similar among vertebrates.

The vertebrate hair cell is at the basis of all vestibular and auditory sensory systems, as well as of the lateral line. Its origin can be dated back at least to the earliest vertebrates (agnatha, jawless fishes) if not to the earliest chordates (Coffin et al. 2004, Manley and Ladher 2008). The first inner ear with hair cells was found in Myxines (hagfishes), the sister group of vertebrates. The ears of hagfishes have one single semicircular canal with two crista ampulares and a sensory epithelium (Jarvik 1980). The lampreys (Petromyzon and Lampetra) are the living representatives of the 
earliest vertebrates and they possess an ear with two semicircular canals and a macula communis that is partially differentiated into several regions (Loewenstein et al. 1968). The functions of the ears in lampreys or hagfishes are not known, but it is assumed that they serve the detection of linear acceleration and gravity.

Three semicircular canals, each oriented in a different plane, and three separated otolith organs were first found in the ears of elasmobranchs and the earliest bony fish. It was suggested that hearing arose from the same structures as vestibular systems (van Bergjick 1967, Ladich and Popper 2004). The first evidence for hearing and sound sensitivity to sound of the inner ear was found in elasmobranchs (Loewenstein and Roberts 1950, Myrberg et al. 1972, Nelson and Johnson 1976, Corwin 1981). Therefore it seems plausible that elasmobranchs most likely show similar coding strategies to ancestral bony fish and teleosts. The significance of hearing in Agnatha still remains to be investigated.

Evolution of coding strategies in vertebrates

Spectral analysis in A. fulvescens as well as in teleosts is probably based on temporal as well as rate coding. All afferents strongly phase-coupled to the stimulus waveform within the frequency range they detected in A. fulvescens. Primary afferents can encode the frequency of a stimulus through phase locking since the information of the stimulus waveform is preserved in the times between spikes. Since phase-locking to lower frequencies (up to about $4 \mathrm{kHz}$ ) is common in all vertebrate 
auditory systems, temporal coding for frequency seems to be a primitive neural code for hearing which occurred early in vertebrate history (Popper and Fay 1999).

A code based on spike rate relying on filters centered on a few different frequencies may not require tonotopicity but central neurons that can encode the population response of a few afferents with different filter shapes. Such a code may be the most primitive rate code for spectral analysis in vertebrates (the ancestral condition).

Throughout the course of evolution, auditory systems developed a greater sensitivity and a greater frequency response range. Encoding a wider spectrum of frequencies was then achieved by breaking up the spectrum into a continuum of narrow ranges, each of which was encoded by a number of hair cells arranged in a tonotopic organization (however tonotopicity is not necessary for frequency coding). Tonotopicity was achieved by systematically varying anatomical (e.g. cell morphology, height of stereovillar bundles) and/or electrophysiological (e.g. ionchannel characteristics) properties of the hair cells and then became a fundamental principle underlying the organization of tetrapod hearing organs.

During the evolution of most groups of amniotes, auditory epithelia (e.g., the basilar papilla in birds, reptiles, and mammals) elongated to a greater or lesser extent independently in all groups (Manley and Clack 2004). Micromechanical tuning became increasingly important during specializations for responses to increasingly high frequencies (lizards up to $8 \mathrm{kHz}$, birds up to $14 \mathrm{kHz}$, mammals up to $100 \mathrm{kHz}$ ) and mechanisms of active amplification improved sensitivity overcoming resistances to movement for hair cells in the inner-ear fluids - just to name a few important 
developments in birds, reptiles, and mammals (for more detail refer to Manley and Clack 2004).

By comparing parameters tested in A. fulvescens with other vertebrate auditory systems, this study has attempted to bring out some of the important commonalities and differences between fish auditory and tetrapod auditory systems (mostly aspects related to coding and the peripheral auditory nervous system). This study sought to improve the understanding on the origin, the underlying mechanisms, and the development of frequency and intensity coding (or even hearing) throughout vertebrate evolution. 

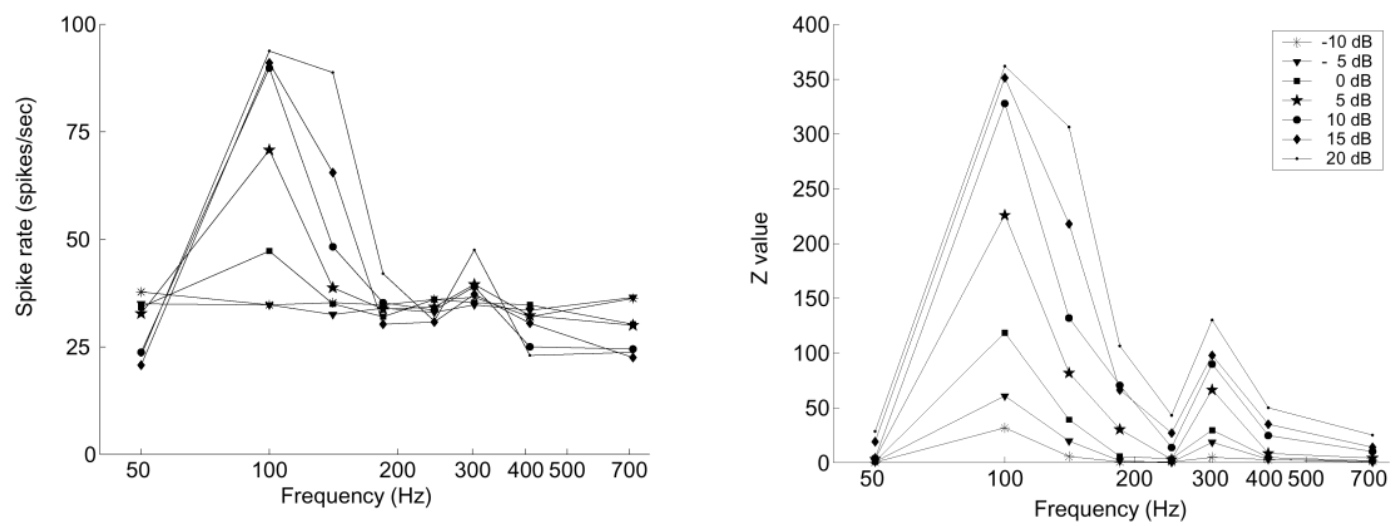

Fig. 2.1: Example of an isolevel frequency function plotted for unit pp9 using two different measures of response strength: spikes per sec (left) and $\mathrm{Z}$ value (right). PP9 has a spontaneous rate of 34 spikes/sec, which leads to a smaller dynamic range during sampling at the same levels to a signal noise detection at a higher threshold when choosing spike rate as the measure of response strength. 


\section{ff11}
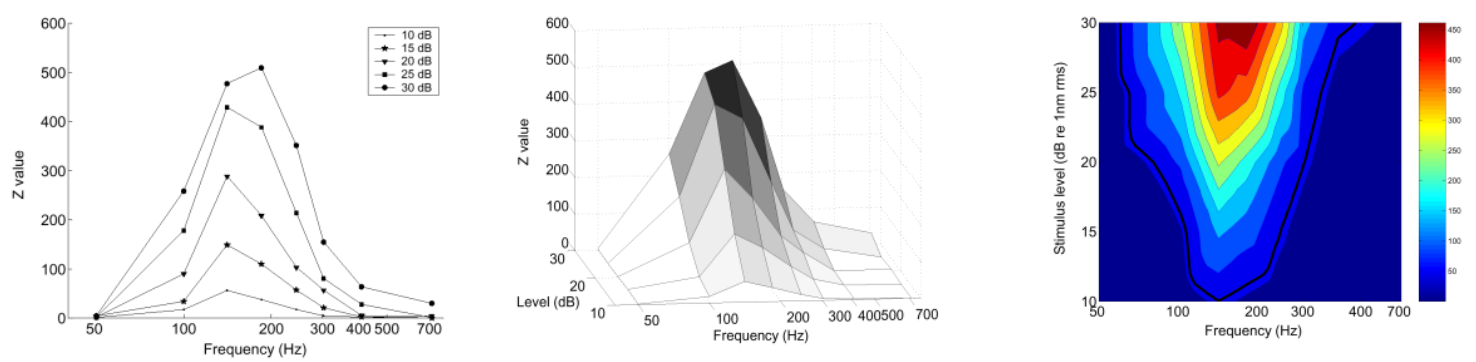

qq5
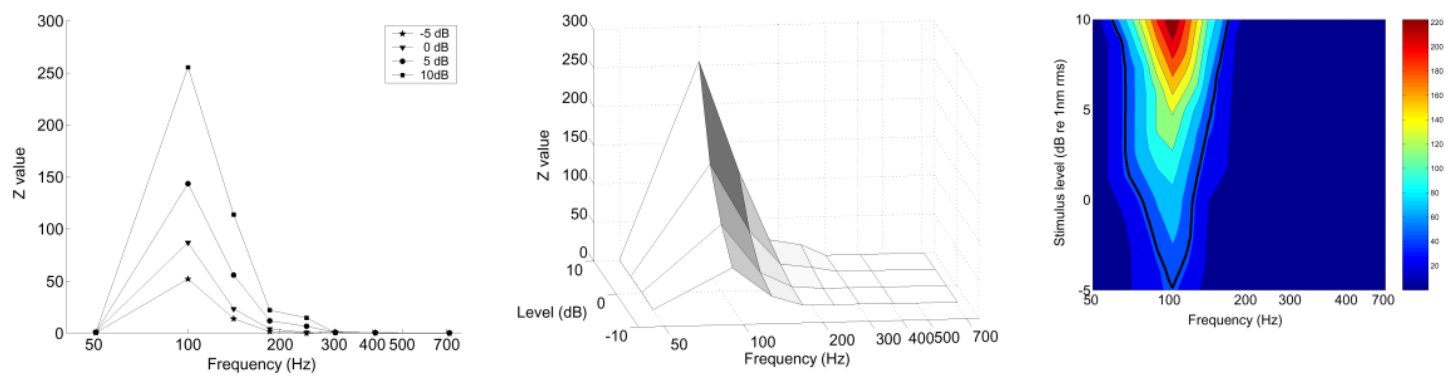

ss11
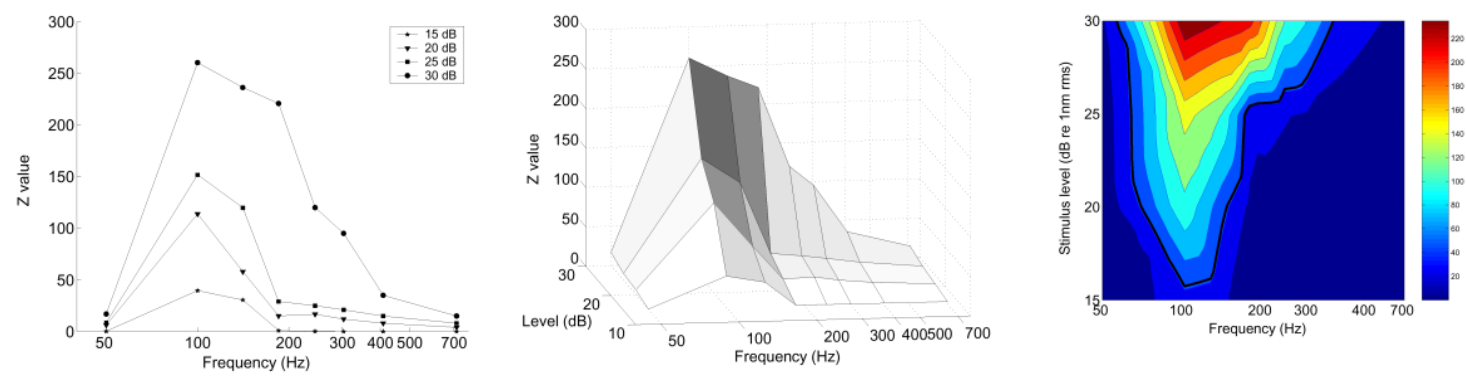

\section{tt3}
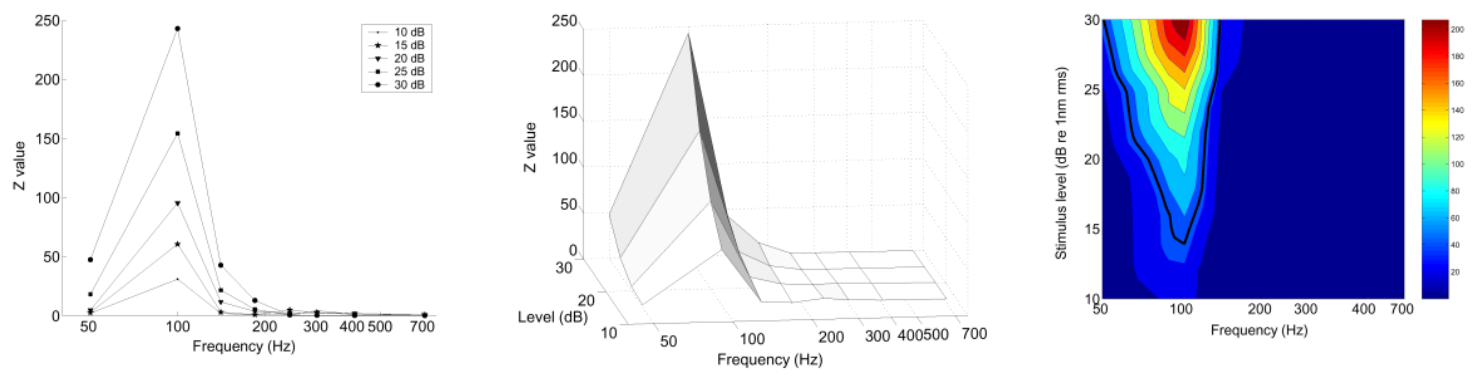
Fig. 2.2: Frequency responses of four units. For each unit isolevel frequency response functions at different levels and a contour plot are plotted. The black line in the contour plot outlines the tuning curve (criterion: $Z=50$ ). Color code to the right of contour plot indicates $\mathrm{Z}$-values (with areas containing more yellow or red components reflecting higher Z-values). ff11: $\mathrm{BF} / \mathrm{CF}=141 \mathrm{~Hz}$ (note the shift of $\mathrm{BF}$ to $185 \mathrm{~Hz}$ at $30 \mathrm{~dB}$ ), BW $(10 \mathrm{~dB}$ re threshold $)=236 \mathrm{~Hz}, \mathrm{Q}_{10 \mathrm{~dB}}=0.57$, Background rate $=15$ spikes $/$ sec. qq5:

$\mathrm{BF} / \mathrm{CF}=100 \mathrm{~Hz}, \mathrm{BW}(10 \mathrm{~dB}$ re threshold $)=79 \mathrm{~Hz}, \mathrm{Q}_{10 \mathrm{~dB}}=1.3$, Background rate $=20$ spikes/sec. ss1: $\mathrm{BF} / \mathrm{CF}=100 \mathrm{~Hz}, \mathrm{BW}(10 \mathrm{~dB}$ re threshold $)=125 \mathrm{~Hz}, \mathrm{Q}_{10 \mathrm{~dB}}=0.83$, Background rate $=43$ spikes/sec. tt3: $\mathrm{BF} / \mathrm{CF}=100 \mathrm{~Hz}, \mathrm{BW}(10 \mathrm{~dB}$ re threshold $)=64$ $\mathrm{Hz}, \mathrm{Q}_{10 \mathrm{~dB}}=1.61$, Background rate $=30 \mathrm{spikes} / \mathrm{sec}$. 
A
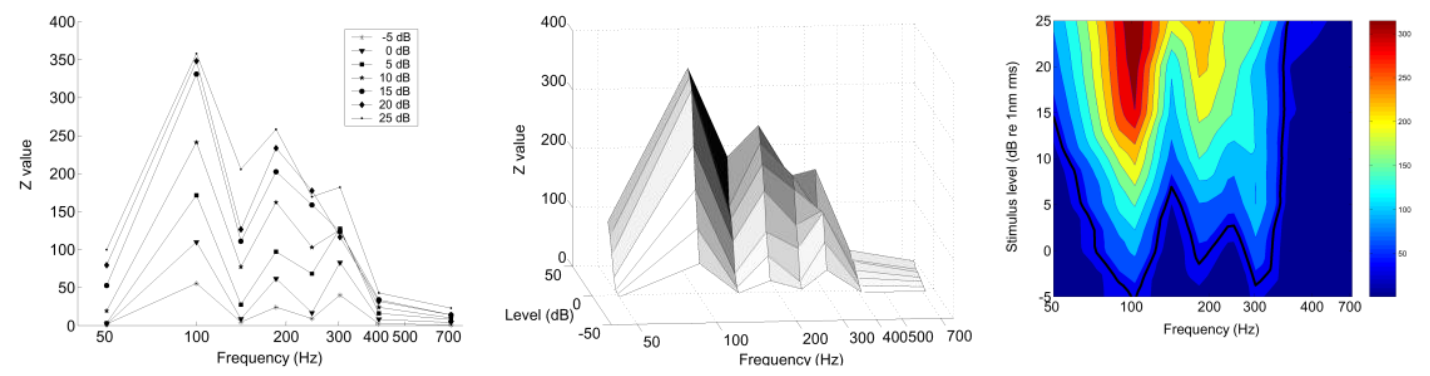

B

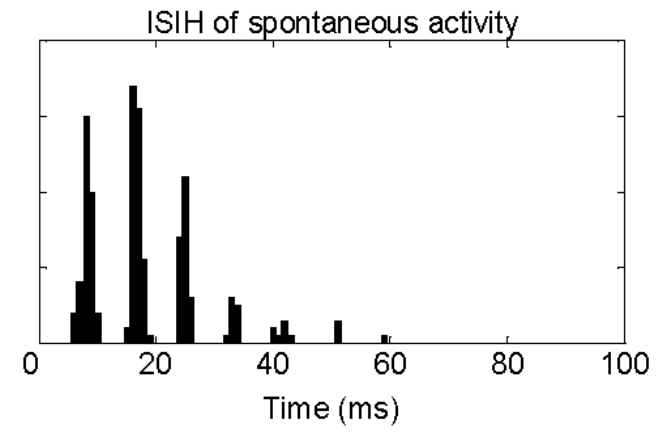

C
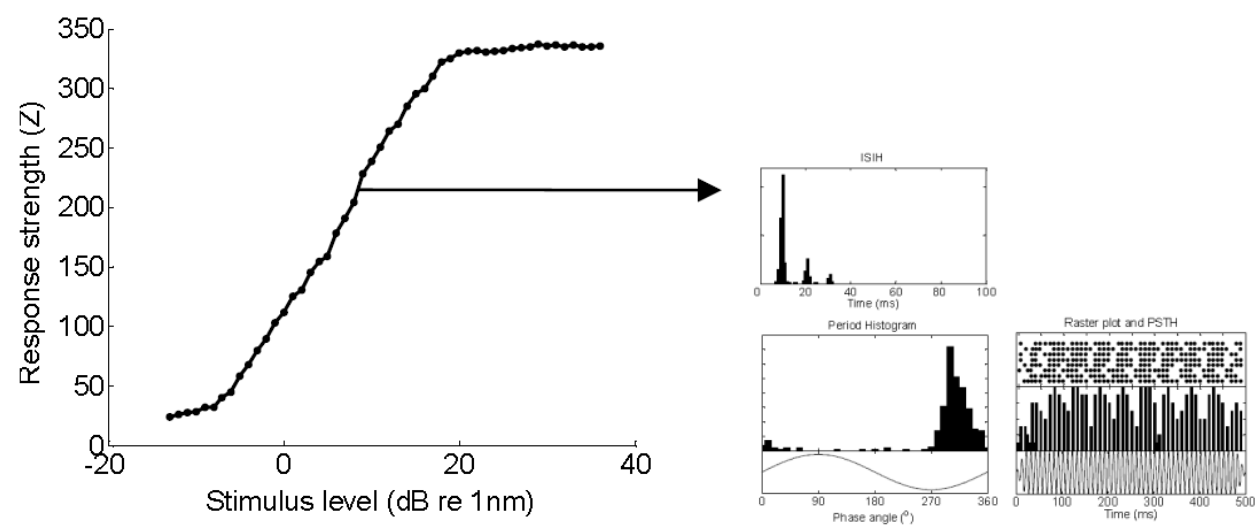

Fig. 2.3: A: Isolevel frequency function of unit tt7 obtained at seven levels showing three peaks $(100,185,303 \mathrm{~Hz})$ and contour plot. B: ISIH of the spontaneous activity of unit tt7 with Poisson distribution. Background rate was 54 spikes/sec. C: Response versus level function of unit $\mathrm{tt} 7 \mathrm{obtained}$ at $100 \mathrm{~Hz}$ (left). Response pattern determined for unit tt7 at $100 \mathrm{~Hz}$ (right); from top to bottom: raster plot and PSTH, ISIH, and PH. Note: all graphs indicate that this was a single unit recording. The unit is strongly phase coupled as the intervals of the ISIH correlate with the multiple integers of the stimulus frequency $(100 \mathrm{~Hz})$. 
A

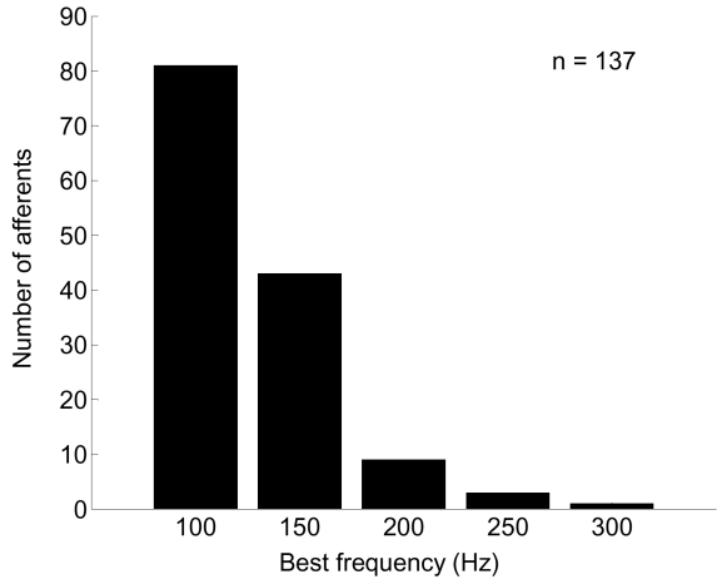

B

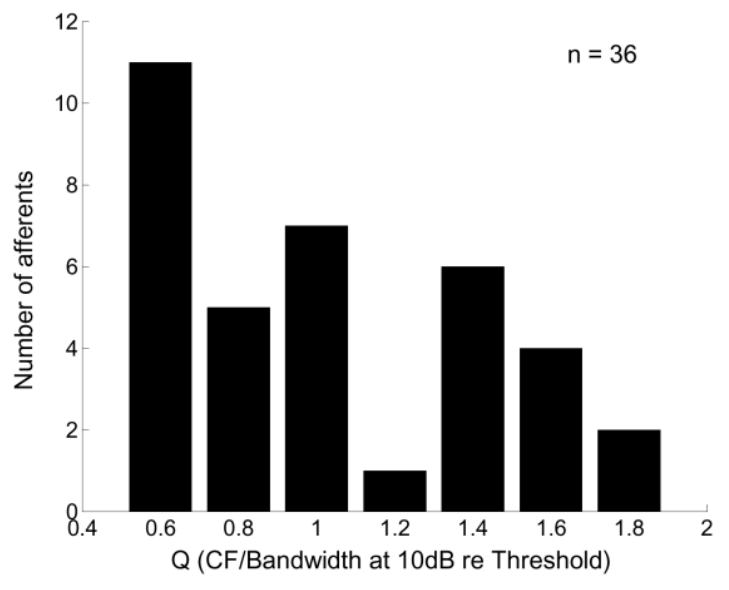

Fig. 2.4: A: Distributions of CFs of mono-peak functions. Bin size is $50 \mathrm{~Hz}$. B: Sharpness of 36 afferents determined from tuning curves: as BW at $10 \mathrm{~dB}$ above threshold at $\mathrm{CF}$ (left, bin width: $20 \mathrm{~Hz}$ ), and $\mathrm{Q}_{10 \mathrm{~dB}}(\mathrm{CF}$ divided by $\mathrm{BW}$ at $10 \mathrm{~dB}$ above threshold) (right, bin width: 0.2). 

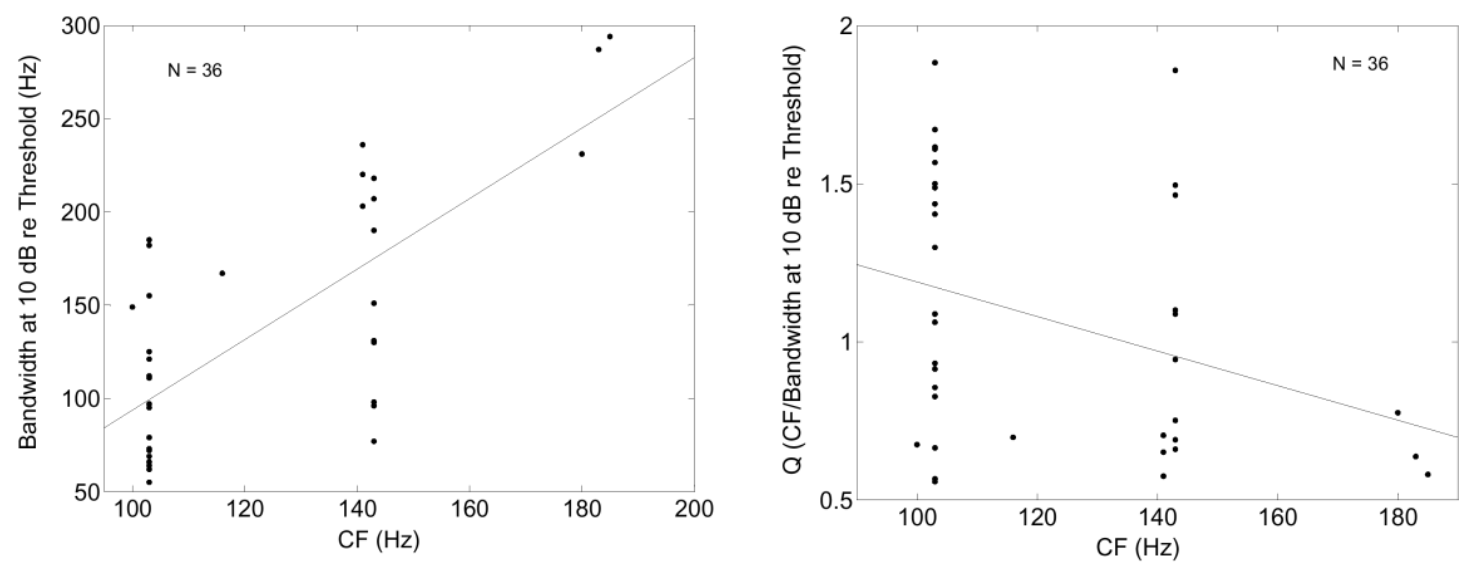

Fig. 2.5: Sharpness of tuning curves decreased with CF. Left: BW at $10 \mathrm{~dB}$ above threshold at $\mathrm{CF}$ is plotted as a function of $\mathrm{CF}$ for 36 afferents $(\mathrm{R}=+0.73, \mathrm{n}=36$, two tails). Right: $\mathrm{Q}_{10 \mathrm{~dB}}$ is plotted as a function of $\mathrm{CF}$ for 36 afferents $(\mathrm{R}=-0.34, \mathrm{n}=36$, two tails). 


\begin{tabular}{|c|c|c|c|c|c|c|c|c|c|}
\hline & $\begin{array}{c}\mathrm{BF} / \mathrm{CF} \\
\mathrm{N}\end{array}$ & $\begin{array}{c}\mathrm{BF} / \mathrm{CF} \\
\mathrm{R}\end{array}$ & $\begin{array}{c}\mathrm{p}- \\
\text { value }\end{array}$ & $\begin{array}{c}\text { SR } \\
N\end{array}$ & $\begin{array}{c}\text { SR } \\
\text { R }\end{array}$ & $\begin{array}{c}\mathrm{p}- \\
\text { value }\end{array}$ & $\begin{array}{l}\text { Thres } \\
\text { h }\end{array}$ & $\begin{array}{c}\text { Thresh } \\
\text { R }\end{array}$ & $\begin{array}{c}\mathrm{p}- \\
\text { value }\end{array}$ \\
\hline $\begin{array}{l}\mathrm{BW}_{10 \mathrm{~dB}} \\
\text { (re CF) }\end{array}$ & 36 & +0.728 & 0.000 & 33 & -0.402 & 0.020 & 35 & -0.037 & 0.833 \\
\hline $\begin{array}{l}\mathrm{Q}_{10 \mathrm{~dB}} \\
\text { (re CF) }\end{array}$ & 36 & -0.339 & 0.043 & 33 & +0.302 & 0.089 & 35 & +0.053 & 0.762 \\
\hline $\begin{array}{l}\text { Sprate } \\
\text { (re BF) }\end{array}$ & 142 & -0.216 & 0.010 & & & & & & \\
\hline
\end{tabular}

Table 2.1: Overview of correlations of parameters; Abbreviations: $N=$ number of units, $\mathrm{R}=$ correlation coefficient; $\mathrm{SR}=$ Spontaneous (background) rate, Thresh = Threshold, $\mathrm{BW}_{10 \mathrm{~dB}}=$ bandwidth obtained at $10 \mathrm{~dB}$ above threshold at $\mathrm{CF}$ for tuning curves, $\mathrm{Q}_{10 \mathrm{~dB}}=\mathrm{CF}$ divided by $\mathrm{BW}_{10 \mathrm{~dB}}$, $\mathrm{p}$-value (the value had to be larger than 0.05 if the correlation was considered to be significant; two tailed test). 
A
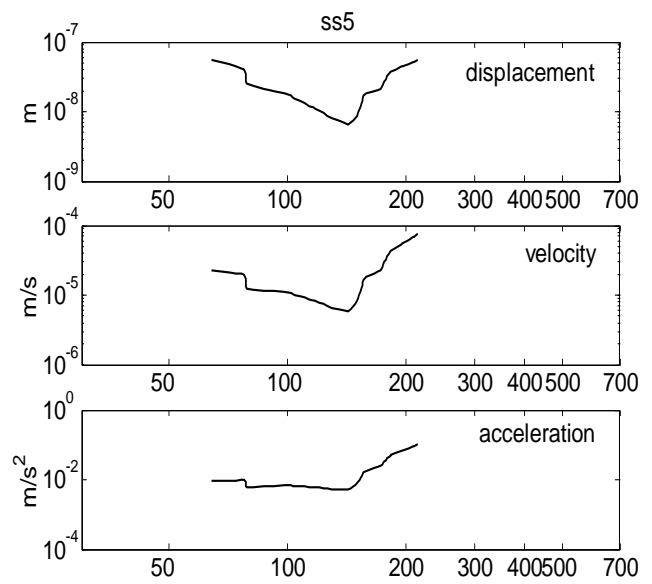

B
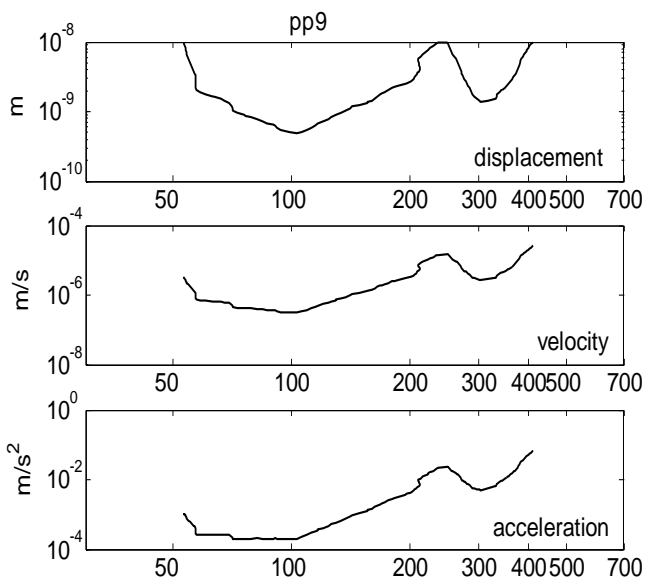

C
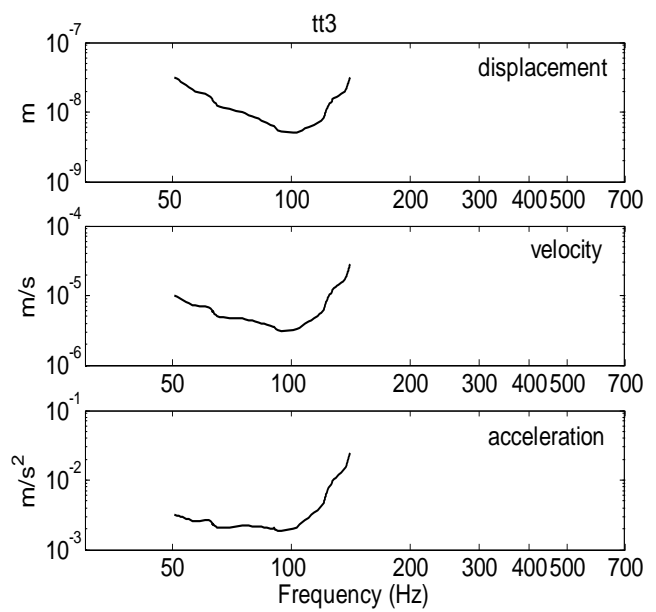
Fig. 2.6: The tuning curves of three different units ss5 (A), pp9 (B), and tt3 (C) with different shapes or CFs were plotted in three different ways: as $\mathrm{dB}$ re $1 \mathrm{~nm}$ displacement (top), $\mathrm{dB}$ re $1 \mathrm{~nm} / \mathrm{s}$ velocity (middle), as $\mathrm{dB}$ re $1 \mathrm{~nm} / \mathrm{s}^{2}$ acceleration - as a function of frequency. All units turned despite of their different shapes into low pass filters when converted from displacement to acceleration. 
A

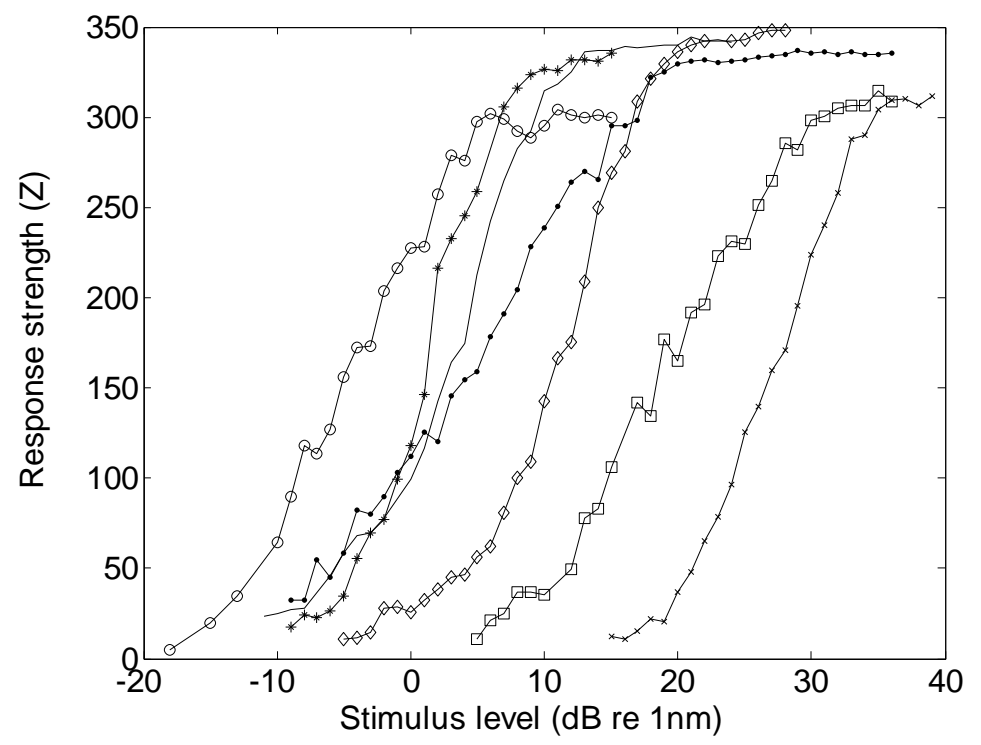

B

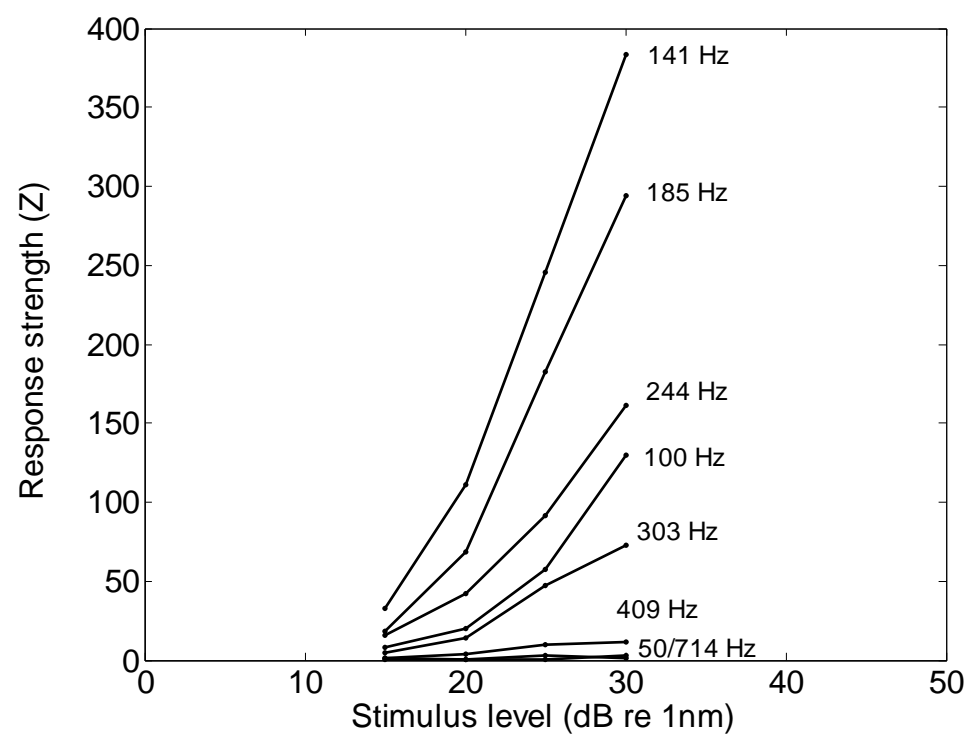

Fig. 2.7: A: Response level functions obtained at the BF of 7 units with a wide variety of thresholds. All functions are monotonically increasing up to a saturation point. The dynamic range and slope also varies between units. B: Response level functions of unit ff 3 with $\mathrm{BF}=141 \mathrm{~Hz}$ at various frequencies. The steepest slope occurs at the BF and steepness decreases with deviation from BF (at frequencies lower, as well as higher than $\mathrm{BF}$ ). 
A

ISIH of Spontaneous activity

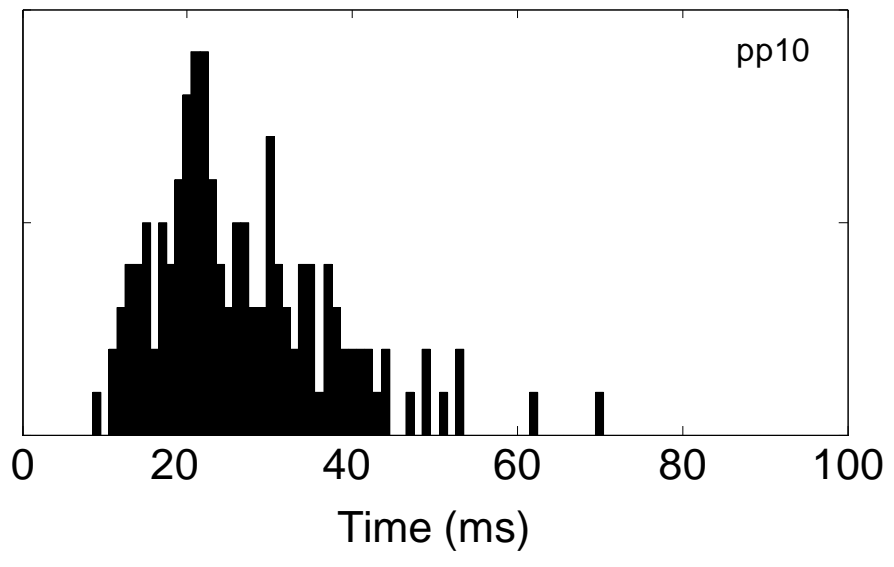

B

ISIH of spontaneous activity

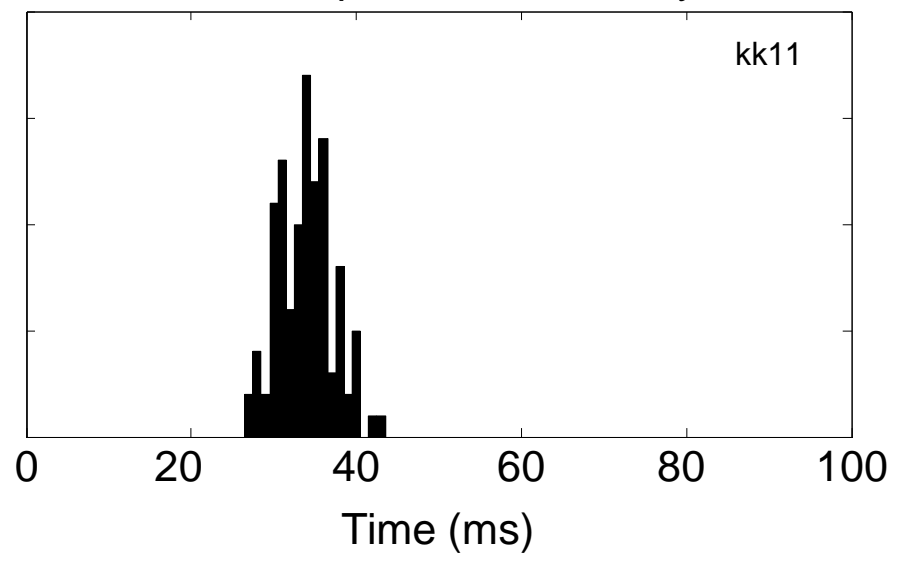

Fig. 2.8: ISIHs of spontaneous (background) activity for two examples. A: unit pp10 showing a Poisson distribution of inter spike intervals - representing $63 \%$ of all investigated frequency-responding units. B: unit kk11 having a Gaussian distribution of inter spike intervals representing $37 \%$ of all investigated frequency-responding units. Spontaneous rate: pp10: 37.5 spikes/sec, kk11: 30 spikes/sec. 
A

Raster plot and PSTH

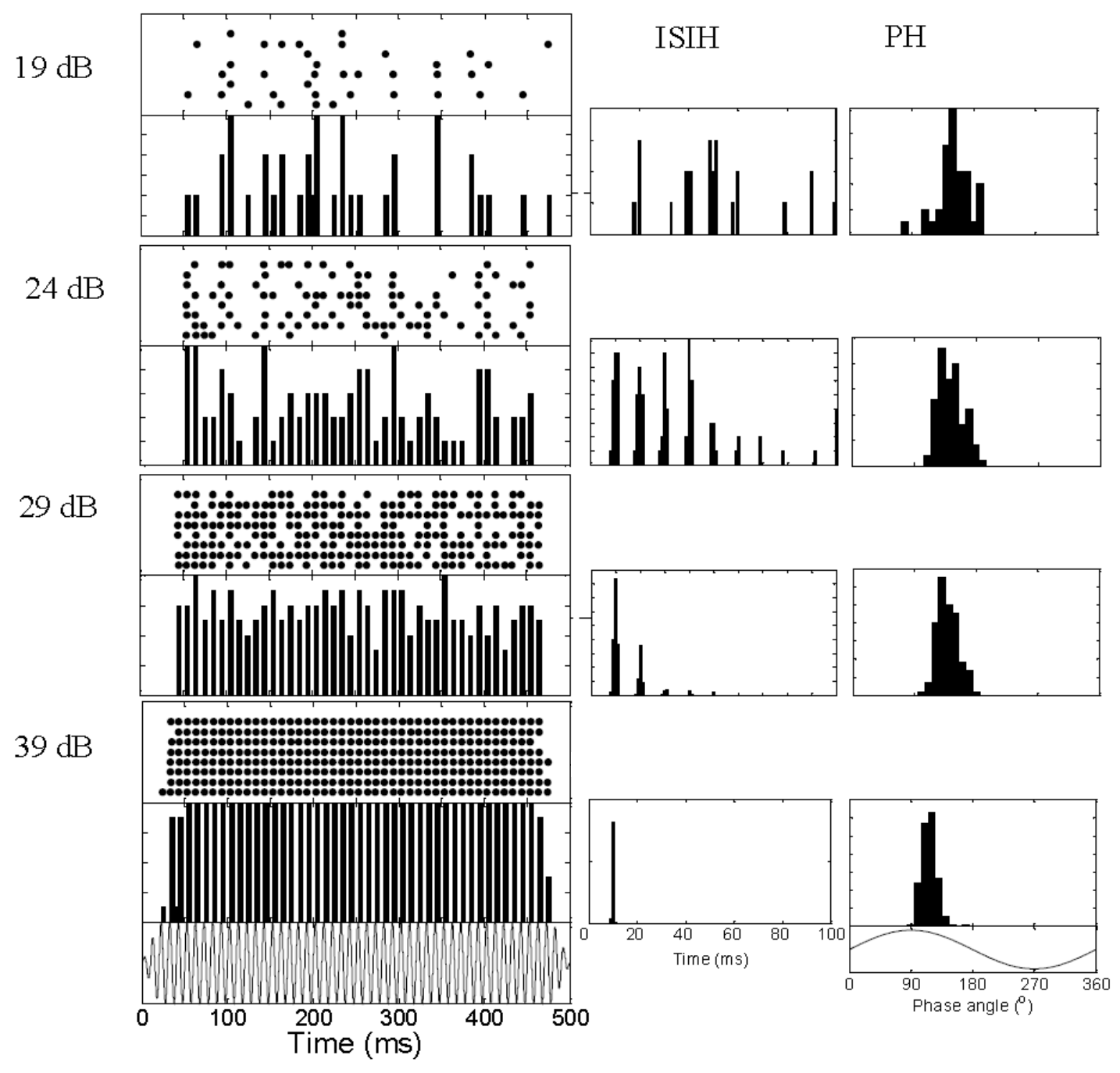


B

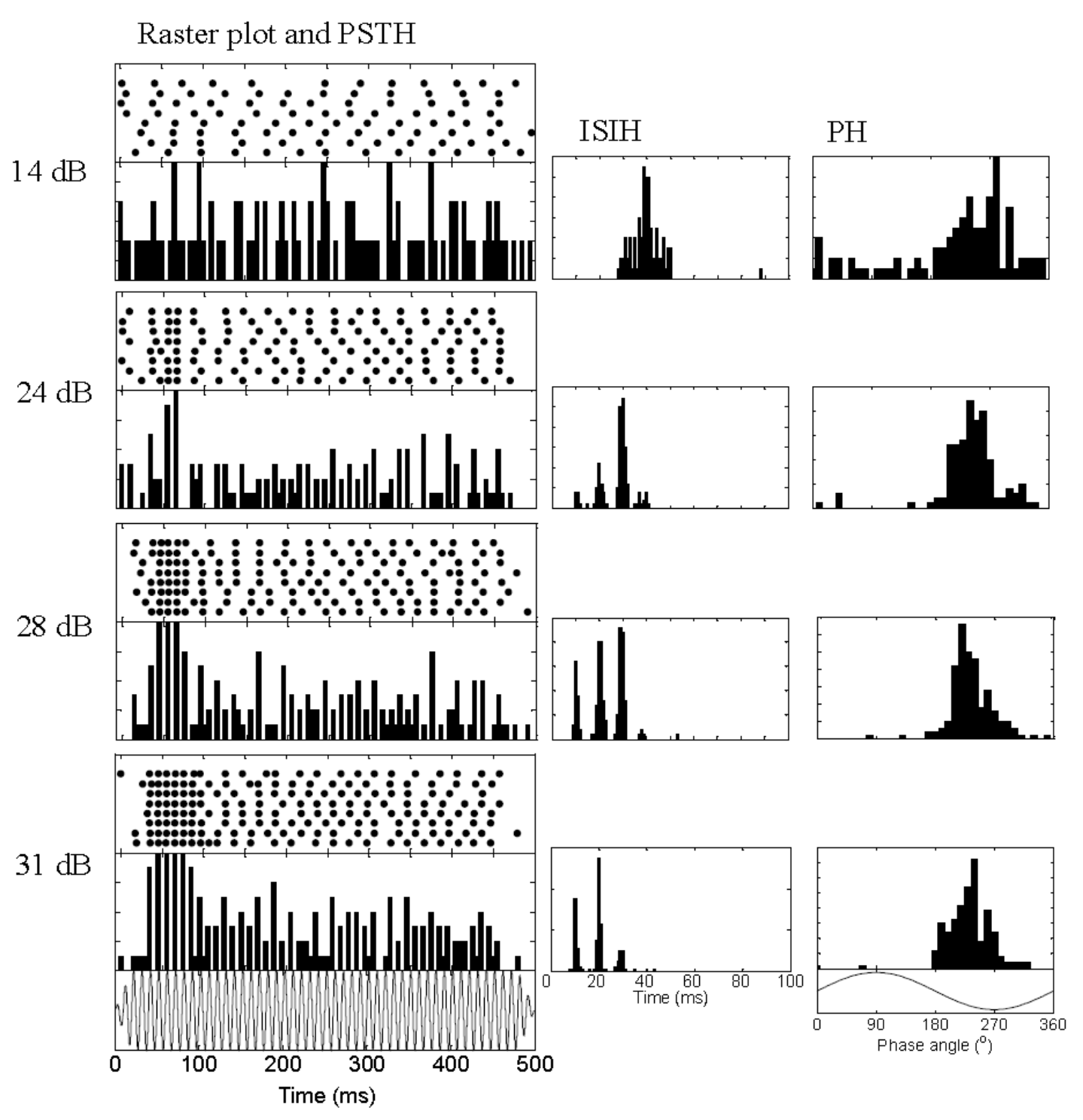


C Raster plot and PSTH
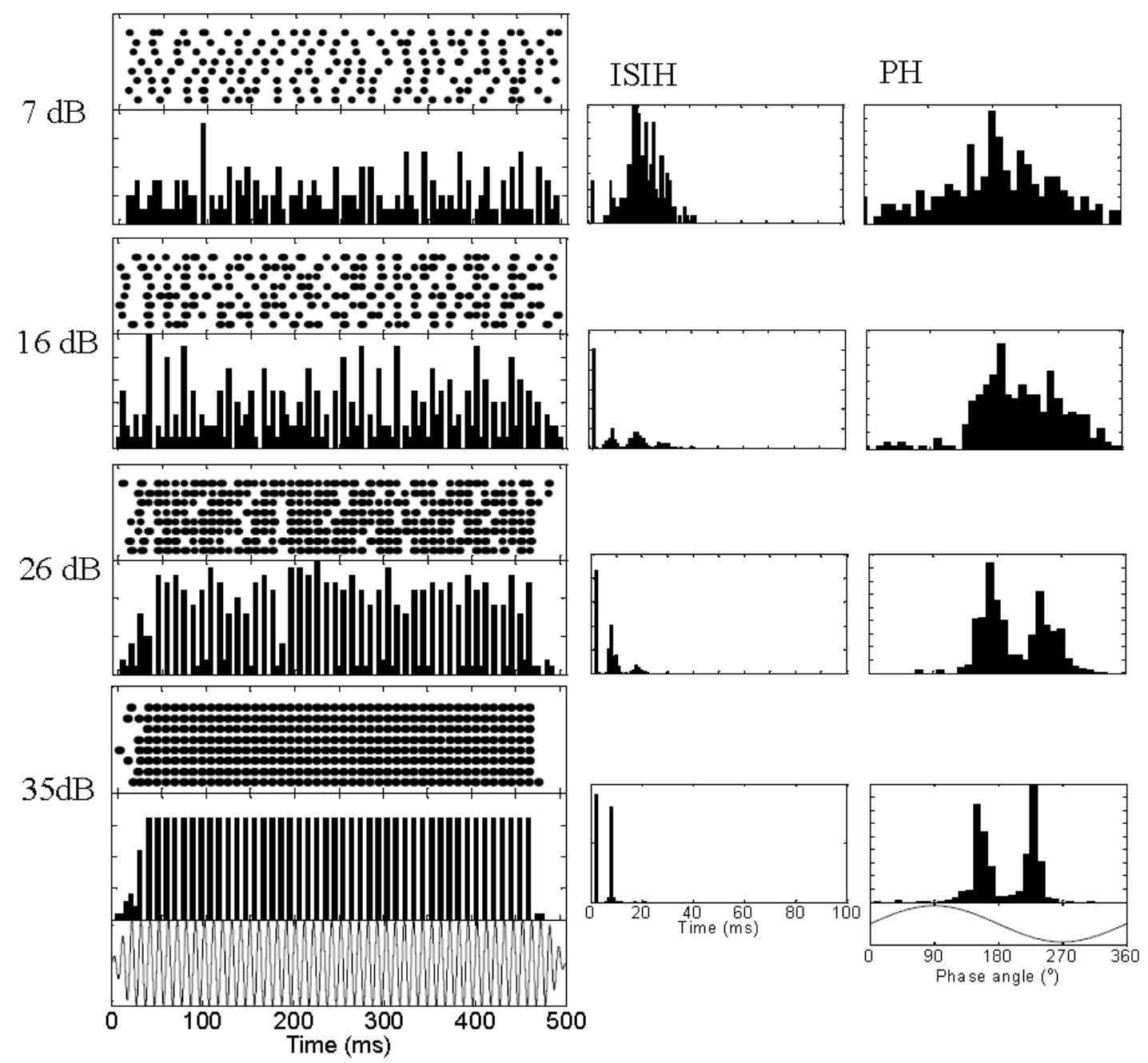

Fig. 2.9: Examples of response patterns; from left to right: raster plot and PSTH ( $1^{\text {st }}$ row), ISIHs ( $2^{\text {nd }}$ row $), \mathrm{PH}\left(3^{\text {rd }}\right.$ row). Each plot is presented at 4 different stimulus levels to show the effect of level on the response pattern. A: Example of unit uu8 showing a tonic response pattern to sinusoidal stimulation at $100 \mathrm{~Hz}$. Background rate: 0 spikes/sec. Threshold: $18 \mathrm{~dB}$ re $1 \mathrm{~nm}$. B: Example of unit pp7 showing a phasic tonic response pattern to sinusoidal stimulation at $100 \mathrm{~Hz}$. Background rate: $23 \mathrm{spikes} / \mathrm{sec}$. Threshold: $13 \mathrm{~dB}$ re $1 \mathrm{~nm}$. C: Example of a phase locked bursting unit (ss10) to sinusoidal stimulation at 100 Hz. Background rate: 43 spikes/sec. Threshold: $6 \mathrm{~dB}$ re $1 \mathrm{~nm}$. 


\section{CHAPTER III}

\section{Coding of sound direction in the auditory periphery of the lake sturgeon, Acipenser fulvescens}

\section{Introduction}

The detection and localization of sound sources in a complex acoustic environment is important for an animal's survival. This information is needed for a variety of behavioral responses, such as escape from predators or approach towards conspecifics. For an animal to make an appropriate behavioral response, its auditory system needs to locate and segregate the range of sound sources it detects into individual auditory events or objects. As a consequence, sound source localization is an important component of source segregation and auditory scene analysis (e.g., Bregman 1990, Fay and Popper 2000, Lewis and Fay 2007). Auditory systems of all vertebrate taxa (e.g., reviewed in Fay and Feng 1987, Fay 2005) as well as of some invertebrates (e.g., reviewed in Robert 2005) have mechanisms to determine the location of a sound source.

The ears of terrestrial vertebrates are sensitive to sound pressure, which in contrast to the particle component of sound cannot convey directional information. Thus, sound source location in terrestrial vertebrates is centrally computed by comparing differences in arrival times, phase, and intensity between the two ears. In aquatic environments, the higher impedance of water results in higher propagation velocities (about 4.5 times higher than in air) of sound waves reducing the differences 
in arrival time and phase at the two ears of a fish. In addition, the small distance between ears relative to the long wavelength of sounds that fish can detect, makes it unlikely that fish use interaural intensity difference cues.

This insight into physical constraints for sound source location under water initially created a debate as to whether fish can localize sound, because it was assumed that fish ears function as sound pressure receivers (v. Bergeijk 1964). In addition, behavioral experiments on sound source location in fish differed in their conclusions therefore leading to controversy about the general capability of fish to locate a sound source (e.g., von Frisch and Dijkgraf 1935, Kleerkoper and Chagnon 1954, Myrberg et al. 1969, Schuijf et al. 1972, Popper et al. 1973; see also reviews by Fay 2005, Sand and Bleckmann 2008).

In contrast to behavioral experiments, studies on the mechanisms possibly underlying sound source location in fish provided less ambiguous insights. The current understanding on the peripheral mechanisms for sound source location is based on the notion that fish ears are mainly adapted to detect the particle motion component of sound (instead of pressure) functioning as inertial accelerometers (de Vries 1950). Particle motion is a vector quantity and potentially carries information about the magnitude and axis of a sound source (Rogers and Cox 1988). Fish (Fay 1984, Edds-Walton and Fay 1999) and some insects (e.g., caterpillars, Tautz 1977, or crickets, Gnatzy and Tautz 1980) that have evolved ears that are suited to detect the particle motion component of sound, are thus potentially able to use this information to detect and locate a sound source. 
The inner ear in fishes (bony and cartilaginous fishes) includes three otolithic end organs (saccule, lagena, utricle), three semicircular canals with their associated crista ampularis, and the macula neglecta. Hair cells in the otolith organs form orientation groups (each with a different orientation) in which the ciliary bundles (including the kinocilium) of each hair cell point approximately in the same direction. Schuijf and Buwalda (1975) hypothesized that the morphologically and physiologically polarized sensory hair cells in each otolith organ (Popper 1976, 1977; Dale 1976) may be innervated by directionally selective primary afferents, representing the axis of particle motion ("vector theory"). Neurophysiological evidence supporting the vector theory was obtained by results using a shaker table which was used to move the fish along various axes during recordings from the eighth nerve, thereby stimulating the fish by the particle motion component of sound (Hawkins and Horner 1981, Fay 1984, Fay and Edds-Walton, 1997a). Results showed that eighth nerve afferents responded to variations in stimulus axes in a cosine-like manner with best directions that are the result of the hair cell orientation of the epithelium that they innervated and the orientation of the epithelium in space (Fay 1984, Fay and Edds-Walton 1997a, Lu et al. 1998, Lu and Popper 2001). Existing studies of the mechanisms of sound source location have been conducted in a few species: Gadus morhua (atlantic cod; Hawkins and Horner 1981), Carassius auratus (goldfish; e.g., Fay 1984, Meyer et al. 2004), Opsanus tau (oyster toadfish; e.g., Fay and Edds-Walton 1997a), Porichthys notatus (plainfin midshipman; Weeg et al. 2002), and Dormitator latifrons (sleeper goby; e.g., Lu et al. 
1998, 2003, 2004). All those studies have been conducted in teleosts (modern bony fishes).

The current study is the first to examine directional responses of eighth nerve afferents in a non-teleost bony fish. Acipenser fulvescens, the lake sturgeon (Rafinesque 1817), belongs to the ray-finned fishes or Actinopterygians. While the majority of Actinopterygians are teleosts (more than 26,000 living species, Nelson 2006), most non-teleost Actinopterygians are extinct (Liem et al. 2001) and encompass only 48 extant species. Of these, 25 are sturgeon (family: Acipenseridae), while two species belong to the sister group, the paddlefishes (Polyodontidae) together forming the order Acipenseriformes.

The Acipenseriformes are known for a long phylogenetic history dating back about 200 million years (Grande and Bemis 1996). Interest in the sensory capabilities of Acipenseriformes has grown (e.g., Sillman et al. 2007) due to their ancient heritage, their migratory behavior (Kynard 1997), and their substantial economic importance. In addition, the threatened or endangered status of many of these species indicates that there may be only limited time left to study these organisms (Krieger et al. 2000).

Studies on non-teleost Actinopterygian groups make important contributions to the evolution of vertebrate hearing since they provide insight into mechanisms of signal processing in an ancestral group. The comparison of data obtained from nonteleost Actinopterygian groups with results from teleost studies helps to find out which character traits are evolutionary ancestral and which are derived (Wilez 1981, Northcutt 1985, 1986, Bolker 2004). Physiological studies on the mechanism for 
directional hearing in any other ancestral group of fishes (cartilaginous, jawless, or lobe finned fishes) have not been conducted before (except for one study in sharks, Casper and Mann 2007). In summary, by selecting an ancestral bony fish (phylogenetically very different from teleosts), this study seeks to add to the understanding on the mechanisms for sound source location and the origin of directional hearing in vertebrates.

In this study we investigated the responses of primary eighth nerve afferents (innervating the saccule and lagena) of A. fulvescens to directional stimuli provided by movement of the fish along defined axes in three dimensions (thereby simulating the particle motion component of sound). By choosing a non-teleost bony fish (phylogenetically very different from teleosts), this study seeks to contribute to the understanding on the peripheral mechanisms of sound source determination in fish and to the evolution of directional hearing in vertebrates.

\section{Material and Methods}

\section{Animal preparation}

Recordings from twenty-one juvenile A. fulvescens (between one and two years old) were used in this study. Their body sizes ranged from 25 to $37 \mathrm{~cm}$ in total length and from 21 to $28 \mathrm{~cm}$ in standard length (body masses: 45 to $115 \mathrm{~g}$ ). They were obtained from the Wild Rose fish hatchery of the Wisconsin Department of Natural Resources and housed in 45-gallon tanks containing aerated water filtered by external filters. Animals were kept at 17 to $18{ }^{\circ} \mathrm{C}$ on a $12 \mathrm{~h}$ light $/ 12 \mathrm{~h}$ dark cycle, and 
were fed with frozen krill (Jehm Co., Inc.). The University of Maryland Institutional Animal Care and Use Committee approved all experimental procedures.

At the start of an experiment, fish were lightly anesthetized in a bath containing $0.05 \%$ buffered 3-aminobenzoic acid methane-sulfonate salt (Sigma) dissolved in tank water, immobilized with an intramuscular injection of gallamine triethiodide in goldfish Ringer's solution ( $43 \mu \mathrm{g} \mathrm{g}^{-1}$ body weight), and then transferred to a surgical dish. Aerated fresh water was pumped through polyethylene tubing inserted into the fish's mouth for artificial respiration (flow rate: 100 to 200 $\mathrm{ml} / \mathrm{min}$ ). For analgesia, the skin at the dorsal surface of the head was dabbed with the local anesthetic Lidocaine (2.5\%) (Astra Chemicals GmbH, Wedel, Germany) for 15 min to let it take effect. Afterwards, skin and bony material of the cranium at the level of the entrance of the eighth nerve into the brain was removed and an opening into the underlying cartilage was cut with a fine, sharp knife. Excess fatty tissue and fluids were aspirated away to reveal the brain surface above the eighth nerve, and exposing part of the cerebellum and the medulla. The exposed brain was kept moist with a fluoroinert liquid (FC-77, Sigma) and special care was taken not to damage semicircular canals and all otolith organs of the ear during surgery. This was confirmed when the tissue was dissected after experiments.

Upon completion of the surgery, the fish was transferred to a cylindrical stimulus dish $($ diameter $=23 \mathrm{~cm}$, height $=6 \mathrm{~cm}$ ) filled with water from a filtered water tank reservoir. The animal's mouth was fitted over and clamped to an aluminum respirator tube delivering aerated tank water of 17 to $18{ }^{\circ} \mathrm{C}$ via a Neslab RTE-111 chiller pump and which was rigidly attached to the cylindrical dish. The 
temperature of the water in the stimulus dish corresponded exactly to the temperature of the holding tank of the animal throughout the entire experiment.

\section{Stimulation system}

This stimulus system was developed to measure directional responses in fish to whole-body translatory movement simulating the effects of the particle motion component of underwater sound in a natural environment (Fay 1984, see also Fay and Edds-Walton 1997a, 1997b, Weeg at al. 2002). The stimulus system consisted of five mechanical shakers. Two pairs of vibration shakers (Bruel and Kjaer \# 4810) were mounted orthogonally to one another via metal rods connected with the cylindrical stimulus dish: one pair was mounted front to back and the other in a side-to-side position of the fish. The fish was tightly attached to the dish via a head holder that was especially made for A. fulvescens. These four shakers created linear oscillatory motions of the dish, thereby moving the fish along distinct axes in the horizontal plane. A fifth shaker (Bruel and Kjaer \# 4809) was attached via a rod to the bottom center of the dish and functioned to oscillate the fish vertically. The combined output from the front-back shaker pair and the vertical shaker created motions along distinct axes in the vertical (or midsaggital) plane of the fish. Similarly, the combined output from the front-back shaker pair and the side-side shaker created motions along distinct axes in the horizontal plane of the fish. The entire shaker system was positioned on a Micro-G pneumatic vibration isolation table.

The dish movement was calibrated for each experiment after the fish was positioned in the stimulus dish by use of three orthogonally oriented accelerometers

(Piezotronics, PCB model 002A10, Flexcel). Using a calibration program (Fay 1984), 
the accelerometer-output was used to make adjustments to the phases and amplitudes required for the shakers to create sinusoidal, linear movements of the dish in the horizontal and midsagittal planes.

During recordings (for details see "extracellular recordings"), a search stimulus was presented at $100 \mathrm{~Hz}$. This search stimulus provided the fish with a constantly changing directional stimulation to maximize the chances of detecting a cell with any directional preference. Once a unit was identified, the directional stimuli (at $100 \mathrm{~Hz}$ ) were presented in six azimuthal axes in the horizontal plane at $90^{\circ}, 60^{\circ}$, $30^{\circ}, 0^{\circ}, 330^{\circ}$, and $300^{\circ}$ and six elevational axes in the mid-saggital plane at $0^{\circ}, 30^{\circ}$, $60^{\circ}, 90^{\circ}, 120^{\circ}$, and $150^{\circ}$. If a unit showed directional selectivity, the directional stimulus protocol was repeated at different levels, spontaneous activity was measured, and for as many units as possible, response-level functions obtained and thresholds determined (for definition of threshold, see "data acquisition and analysis").

Signals to the shaker system were digitally synthesized sinusoids, $500 \mathrm{~ms}$ long, with $20 \mathrm{~ms}$ rise and fall times repeated eight times. They were independently read out of the three channels of a 16 bit digital-to-analog converter (Tucker Davis Technologies, TDT, DA) at a $10 \mathrm{kHz}$ sampling rate. Each signal was low-pass filtered at $2 \mathrm{kHz}$, controlled in amplitude by TDT PA4 programmable attenuators, and amplified by a Techron power amplifier (Model 5507). The amplified signals were further attenuated by $32 \mathrm{~dB}$ using a resistor network to improve signal-to-noise ratio at the shaker inputs. 


\section{Extracellular recording}

Fish were left for recovery from anesthesia for about $1 \mathrm{~h}$ before starting electrophysiological experiments. Extracellular recordings were made using $2 \mathrm{M}$ $\mathrm{NaCl}$-filled glass pipettes with resistances between 20 to $50 \mathrm{M} \Omega$. These fine tip resistances were achieved using a micropipette puller (Sutter Instrument Co, P97). To gain access to the posterior ramus of the eighth nerve (innervating the right ear), the brain was carefully retracted to the left side using a self-made plastic retractor, the micropipette positioned on the nerve under visual control, and then advanced through with a remote hydraulic micro drive. The tip of the reference electrode (a silver silver-chloride wire of $1 \mathrm{~mm}$ diameter) was placed in the fluid of the brain cavity. Neural signals were amplified using a DAM 80 (World Precision Instruments) and band pass filtered between 300 to $3000 \mathrm{~Hz}$.

\section{Data acquisition and analysis}

Once action potentials from a single afferent fiber were recorded, responses to different axes (as defined above) were measured. Spikes were discriminated using a single voltage criterion, and spike times were recorded with a $10 \mathrm{kHz}$ sampling rate.

From the spike times recorded during stimulation, period histograms were formed and the coefficient of synchronization $(\mathrm{R})$ was calculated to measure the degree of phase locking (Goldberg and Brown 1969). In order to minimize misinterpretations of R when having a smaller number of spikes (Ns), the Rayleigh statistic $\mathrm{Z}=\mathrm{R}^{2} \mathrm{~N}_{\mathrm{s}}$ was applied to quantify phase locked responses (Batschelet 1981, Fay 1984). The $\mathrm{Z}$ value represented the response magnitude for afferents in this study. 
For units that could be held long enough, response-versus-level functions were determined for stimuli at three orthogonal stimulus axes: $90^{\circ}$ elevation (updown), $90^{\circ}$ azimuth (side-side), and $0^{\circ}$ azimuth (front-back). The threshold for each of the three orthogonal directional stimuli was determined as the stimulus displacement level (dB re: $1 \mathrm{~nm}$ ) which corresponds to $\mathrm{Z}=20$ (the probability of obtaining a $\mathrm{Z}$ value of 20 or more by chance is less than 0.001 ).

To quantify directional preference of individual afferents, the Z-value as a function of stimulus direction was plotted and shown in two polar coordinate plots, one formed using the six defined stimulus axes in the horizontal plane and the other using the six corresponding axes in the mid-saggital plane. These polar plots, or directional response profiles (DRPs), were plotted independently for different stimulus levels. For each plot, the response data at the six angles were plotted twice, once at the nominal axis angle then again at the angle plus $180^{\circ}$. This convention was used because the stimulus was an oscillatory motion along the given axis and neural responses were averaged therefore not defining one preference for one polarity of oscillation over the other. The best axis (BA) in three dimensions were computed from the BA components measured in the horizontal and vertical planes. 


\section{Results}

\section{Anatomy}

\section{Spatial orientation of otolith organs and innervation}

The sensory epithelia of the utricle, saccule, and lagena in A. fulvescens are covered by otoconia embedded in a gelatinous matrix, unlike in teleosts, which possess a solid otolith. The utricle is horizontally oriented while the saccule and lagena are located in one vertically oriented pouch. Most hair cells on the saccule are dorsoventrally oriented, but the anterior part of the saccule bends such that hair cells of that portion are now oriented approximately along the anterior posterior axis (Fig. 3.1). The hair cells of the lagena show more variation with some hair cells being oriented dorsoventrally and some deviating from this orientation slightly (Fig. 3.1). Most areas of the saccular and lagenar epithelia are nearly parallel to the mid-saggital plane as determined from CT scans (computer-assisted tomography scans) that were constructed from cross sections through the head of A. fulvescens (Meyer and Ketten unpublished).

The eighth nerve in A. fulvescens exits the brain and branches off into two major rami, one that innervates the utricle and the anterior and horizontal cristae (anterior ramus) and the other innervating the saccule, lagena, macula neglecta, and the posterior crista (posterior ramus). The posterior ramus runs parallel to saccule and lagena (Fig. 3.1). At the level of the saccule, the ramus sends off many small and very short branches (all more or less parallel to each other), which innervate hair cells of 
the saccule (Fig. 3.1). Then the ramus divides into two major branches closely attached to the pouch, one of them innervates the posterior crista, and the other innervates the lagena and macula neglecta.

Recordings were made from the portion of the posterior ramus that was located most proximal to the brain. It was impossible to access any distal portions of this ramus for recordings due to its tight connection with the pouch containing the saccule and lagena and without severely damaging those epithelia (Fig. 3.1). For referral to certain units and animals, the convention aa, bb, cc was used to designate specific animals and numbers were used to identify units (e.g. "pp11" for unit number 11 recorded from animal "pp").

\section{Physiology}

Neurophysiological recordings were made from single afferents of the right posterior ramus of the eighth nerve in A. fulvescens. A total of 177 single units from 21 animals showed directional selectivity and phase locked to the stimulus waveform. Directional responses were analyzed with respect to best azimuth, best elevation, and best response axis in three dimensions. Eighty-three units were encountered during recordings that showed no phase-locking and no directionality. A small subset of these units (27/83) were considered for data analysis to test the effect of level and frequency on spike rate of these units. 


\section{Background activity and thresholds}

Background activity was defined as spontaneous firing of spikes of units in the absence of an intentional stimulus. Background rates were determined for 159 afferents and ranged from 0 to 142 spikes s $^{-1}$ with a mean of 23 spikes s $^{-1}(\mathrm{SE}=1.9$; Fig. 3.2). Most afferents (91\%, $145 / 159$ tested) were spontaneously active and only 9\% (14/159) had no background rate. Twenty eight percent (44/159) fired with a rate lower than five spikes s ${ }^{-1}$ and 3\% (5/159) had a spike rate higher than 80 spikes s$^{-1}$ (Fig. 3.2).

For 67 units, response-level functions (in $1 \mathrm{~dB}$ increments) were recorded and thresholds determined. Figure 3.3 shows the distribution of displacement thresholds obtained with vertical movement since most units responded best to stimulation at about $90^{\circ}$ in the vertical plane. Thresholds ranged from $-23 \mathrm{~dB}$ to $34 \mathrm{~dB}$ (re: $1 \mathrm{~nm}$ ) with a mean of $9 \mathrm{~dB}$ re: $1 \mathrm{~nm}$, rms (SE: 1.7) corresponding to displacement values of $0.07 \mathrm{~nm}(-23 \mathrm{~dB}), 2.8 \mathrm{~nm}(9 \mathrm{~dB})$, and $50 \mathrm{~nm}(34 \mathrm{~dB})$. Background activity and threshold were significantly correlated in that units with high background activity tended to have low thresholds $(\mathrm{r}=-0.25, \mathrm{n}=65, \mathrm{p}<0.05$, two tails $)$. After removal of two units with very high background activity (outliers, see Fig. 3.4), however, the correlation was not significant $(\mathrm{r}=-0.17, \mathrm{n}=63, \mathrm{p}<0.05$, two tails $)$.

\section{Directional responses}

Directional selectivity was found in 177 afferents. Directionally selective afferents showed a directional response profile (DRP) that approximately resembled a 
cosine function (more or less circular in polar coordinates), with a best axis in the vertical and/or horizontal plane indicating the best axis of a unit (BA).

The DRP-shapes shown in Figure 3.5 are representative for directionally tuned units in A. fulvescens and resemble the DRPs seen in teleost fishes (e.g. Fay 1984, Weeg et al. 2002, Lu et al. 2004, Meyer et al. 2004). Most afferents (76 \%, 135/177) had their best axis in the vertical plane (between $80^{\circ}$ and $100^{\circ}$ ) and did not respond at all to horizontal stimulation (afferents pp11, oo2 in Fig. 3.5). The remainder $(24 \%, 42 / 177)$ showed responses in a cosine-like manner in the horizontal plane (bb13, ff11, mm14) within the afferents dynamic range (ff11). The BAs in the horizontal plane were grouped between $-28^{\circ}$ and $25^{\circ}$ azimuth (26/42). For these afferents, the BAs in the vertical plane usually differed from $90^{\circ}$ (e.g., ff11: vertical plane-BA $=120^{\circ}$ ). There were, however, a few afferents with BAs in the vertical plane at $90^{\circ}$, which had BAs at or near $90^{\circ}$ as well (16/42; mm14 Fig. 3.5).

Figure 3.6 shows two examples of afferents representing extreme cases. Afferent oo9 had a narrow vertical plane DRP within the dynamic range of the unit (5 and $10 \mathrm{~dB}$ ) and lost directionality at the highest level (circle at $30 \mathrm{~dB}$ ). The response to horizontal plane stimuli (DRP with BA at $0^{\circ}$ ) only occurred at the highest levels (at the saturation of the unit).

The DRP of a unit was called "narrow" when the ratio of the $\mathrm{Z}$ at BA and the $\mathrm{Z}$ deviating $\pm 30^{\circ}$ from BA was larger than the ratio of these two Z-values for a cosine-shaped DRP. Six percent (10/177) of afferents had a narrow DRP at lower levels like oo9, but this afferent was the only one loosing directionality at the highest level tested. Afferent mm8 also had a narrow DRP in the vertical plane and the profile 
remained narrow across all levels (even at the highest level: $35 \mathrm{~dB}$ ) as it was the case for three percent $(6 / 177)$ of all afferents.

Non-directional responses

Eighty-three units encountered during recordings did not show any phase locking to the stimulus waveform $(\mathrm{Z} \leq 5)$ and did not respond in a cosine-like manner to directional stimuli. Spike rate as a function of stimulus angle was plotted for a subset of these units (27/83). Thirteen of them fired with an increased spike rate to directional stimuli but they showed approximately the same spike rate to all angles tested (Fig. 3.7 top) and were therefore classified as omnidirectional. In addition, their isolevel frequency response function resembled a low pass filter with a best frequency smaller than $100 \mathrm{~Hz}$ (not shown in Fig. 3.7 top) and their spike rate increased with stimulus level. Fourteen of the non-phase-locked afferents also showed no increase in spike rate to directional stimuli and thus are not classified as omnidirectional.

\section{Best directions in three dimensions}

Figure 3.8 summarizes the distributions of BAs in the horizontal and vertical planes showing uniformity for BAs in the vertical plane (clustered around $90^{\circ}$ ) and some spread of BAs in the horizontal plane. The majority of horizontal plane BAs are found between $-28^{\circ}$ and $25^{\circ}$.

The angular orientations for 161 afferents evaluated for BAs in azimuth and elevation are summarized in spherical space (Fig. 3.9). Although the majority of 
afferents had their best axis in three dimensions at $90^{\circ}$ vertical, the $3 \mathrm{D}$ representations illustrate that there is some spread in the vertical plane (Fig. 3.9 A, C).

There is much less variety in responses of units that respond to stimuli in the horizontal plane (Fig. 3.9 B). Most axes pointed towards $0^{\circ}$ azimuth (Fig. 3.9 B, front back direction). Since major parts of the saccule and lagena are located parallel to the midsaggital plane, a horizontal best axis near $0^{\circ}$ for most afferents was expected. Those afferents that had a BA at $90^{\circ}$ for vertical and horizontal DRPs were not included in the $3 \mathrm{D}$ representation (see discussion for explanation).

Dependence of response strength $Z$ and phase angle on level and spontaneous activity

Response vs. level functions and phase angle vs. level functions for two afferents with different spontaneous activity are plotted in Figure 3.10. Response level functions were obtained at three different directions (front back, side to side, vertical). The shape of the response level functions as shown for unit tt7 was usually independent of direction (for all units), yet thresholds varied for each direction as expected from the DRPs.

There was no significant correlation between slope of the response-level functions and spontaneous rate $(\mathrm{r}=-0.5, \mathrm{n}=11, \mathrm{p}<0.05$, two tails $)$ and dynamic range versus spontaneous rate $(\mathrm{r}=+0.35, \mathrm{n}=10, \mathrm{p}<0.05$, two tails $)$. Phase angle versus level functions were plotted to determine whether the phase angle would change with level or not. For afferent $t \mathrm{t} 7$, that had a lower spontaneous activity $(54.5$ spikes $\mathrm{s}^{-1}$ ), the phase angle decreased with level, whereas the phase angles of afferent tt6 with high spontaneous activity (117 spikes s $\left.^{-1}\right)$ remained constant with increasing 
level. Plotting the slopes (of 10 phase angle versus level functions) as a function of spontaneous rate showed no significant correlation between these parameters $(\mathrm{r}=$ $+0.59, \mathrm{n}=10, \mathrm{p}<0.05$, two tails). However, the phase angle of afferents that had very high spontaneous rates (higher than 70 spikes s$^{-1}$ ) tended to stay constant with level.

\section{Discussion}

Afferents innervating the saccule or lagena in A. fulvescens had cosine-like response profiles indicating directionality although mostly to stimuli in the vertical plane (with a preference near $90^{\circ}$ vertical). Most afferents were spontaneously active and spontaneous activity between afferents was very variable. Afferents had a wide variety of thresholds ranging between 0.07 and $50 \mathrm{~nm}$ displacement. Afferents were highly phase locked to the stimulus, thus information about phase can be conveyed to the central nervous system. However, phase angles varied among afferents and with stimulus level (except for units with spontaneous activity larger than 70 spikes $\mathrm{s}^{-1}$ ). Therefore, it seemed unlikely that phase angle information could be used to resolve a $180^{\circ}$ ambiguity in $A$. fulvescens.

\section{Background activity and sensitivity}

Afferents in A. fulvescens showed high variability in background firing as seen in teleost fishes. Maximum values for A. fulvescens (142 spikes s ${ }^{-1}$ ) were lower than maximum values detected for the lagena (160 spikes s ${ }^{-1}$, Meyer et al. 2004) and saccule in C. auratus (250 spikes s ${ }^{-1}$, Fay and Ream 1986), the saccule in $O$. tau (180 spikes s$^{-1}$, Fay and Edds-Walton 1997a), and the saccule of D. latifrons (162 spikes 
$\mathrm{s}^{-1}$, Lu et al. 1998). Although $28 \%$ of the afferents (44/159) fired with a rate lower than five spikes s${ }^{-1}$, only $9 \%(14 / 159)$ were not spontaneously active in contrast to studies in teleosts (e.g., $45 \%$ of the saccular afferents of D. latifrons showed no background activity; Lu et al. 1998).

Thresholds of afferents are widely distributed. Thresholds were determined at $90^{\circ}$ vertical stimulation (no horizontal angle), since this was the BA for most afferents in A. fulvescens. Thresholds ranged from below $-23 \mathrm{~dB}$ to $34 \mathrm{~dB}$ re: $1 \mathrm{~nm}$, which corresponds to displacements ranging from $0.07 \mathrm{~nm}$ to $50 \mathrm{~nm}$, rms. This wide threshold variation among afferents is equivalent to that for saccular or lagenar afferents of C. auratus (Fay and Ream, 1986; Fay 1984; Meyer et al. 2004), O. tau (Fay and Edds-Walton 1997), and D. latifrons (Lu et al. 1998). A wide variation in thresholds guarantees that sounds of widely different level may be encoded within the unit's dynamic range.

The lowest threshold found in saccular afferents of C. auratus (Fay 1984) and O. tau (Fay and Edds-Walton 1997) was $0.1 \mathrm{~nm}$ for both species. The saccular otolith shapes are very different across these fish species. In addition, the otolith in $O$. tau has a far greater mass than the one in C. auratus (Popper and Coombs 1982) yet, saccular afferents in both species have similar thresholds. The ears in A. fulvescens contain otoconia that appear like compressed or loosely distributed particles (in contrast to a dense otolith in teleosts; Lychakov 1995, Gauldie 1996). Taking together the observations from O. tau, C. auratus, and A. fulvescens, there seemed to be no evidence that otolith mass or shape correlates with or causes lower thresholds. 
The low thresholds found in A. fulvescens and teleost species are not uncommon for auditory systems in vertebrates. For instance, the basilar membrane displacement in the guinea pig is about $0.2 \mathrm{~nm}$ at $0 \mathrm{~dB}$ sound pressure level (Allen 1996) and phase locked responses have been recorded to vibrations as low as $0.1 \mathrm{~nm}$ from afferents innervating the sacculus of Lithobates catesbeianus (American bullfrog; Koyama et al. 1982).

In O. tau, low spontaneous saccular afferents tended to have higher thresholds than high spontaneous afferents (Fay and Edds-Walton 1997a) similar to recordings from cochlear afferents of mammals (reviewed in Ruggero 1992). Such a correlation was not found in D. latifrons (Lu et al. 1998).

In A. fulvescens, the result is unclear. Low spontaneous afferents tended to have higher thresholds but the correlation depended critically on two afferents with very high background activity.

\section{Directional and non-directional afferents}

Directional selectivity was found in $68 \%(177 / 260)$ of the afferents in the sense that the shape of the isolevel response functions to varying directional stimuli of these afferents resembled a cosinusoidal function (dipole) - as seen in teleosts (Hawkins and Horner 1981, Fay 1984, Fay and Edds-Walton 1997a, Lu et al. 1998, Weeg et al. 2002). These cosine-like shapes are similar to the functions expected from single hair cell stimulation (Flock 1964, Hudspedth and Cory 1977).

Some afferents showed deviations from the typical cosinusoidal DRP. These included narrowing of the DRP, either at lower levels within the unit's dynamic range or across all levels. The unit shown in Figure 6 (oo9) had narrow DRPs at levels 
within the unit's dynamic range, changing to cosine like DRPs at saturation-levels of the unit and then becoming omni-directional at the highest stimulus level. At saturation-levels, the unit also showed responses in the horizontal plane with a BA at $90^{\circ}$. A response pattern of this sort might be explained by hair cells that are not exactly oriented at $90^{\circ}$ (vertical) but instead are slightly tilted within the vertical plane. Such hair cells become responsive to front back stimulation only at very high levels. This example illustrates that directionality depended on level. If afferents were stimulated at very high levels, directionality became ambiguous.

Afferents with sharpened DRPs across all levels (mm8, Fig. 3.6) have also been described for a few saccular afferents in P. notatus (Weeg et al. 2002) and lagenar afferents in D. latifrons (Lu et al. 2003). As in P. notatus (Weeg et al. 2002), C. auratus (Fay 1984), and D. latifrons (Lu et al. 1998), some DRPs were found that flattened and broadened as levels increased in A. fulvescens and will be discussed further below (see model fitting DRPs).

Afferents that did not show any phase locking to the stimulus waveform were also found in A. fulvescens. These units (27 investigated/ 83 non-phase-locking) had very low $Z$ values $(Z \leq 5)$ and 13 of them fired with increased spike rates to directional stimulation. These units showed approximately the same spike rate to all angles tested (omni directional) but the spike rate decreased with frequency (lowpass) and increased with stimulus level. Similar responses have been reported for $O$. tau (Edds-Walton et al. 1999, Weeg et al. 2002) and have been categorized as responses from efferent fibers. Anatomical studies in $O$. tau showed that fibers of this response type innervated a much larger area of the saccular epithelium than did units 
showing phase-locked, directional tuning (Edds-Walton et al. 1999). The remainder of the non-phase-locking afferents had no low-pass characteristics and did not change in firing rate with level or direction. These afferents may innervate the posterior crista or macula neglecta.

\section{Coding for sound source location}

The posterior ramus of the eighth nerve in A. fulvescens carries neurons that innervate the saccule and the lagena, the posterior crista, and the much smaller macula neglecta. Afferents with directional tuning included in this study, most likely received their input from the saccule or the lagena since their responses show strong resemblance to the shape of DRPs recorded from afferents innervating otolith organs in teleosts. The macula neglecta and the posterior crista do not contain otoliths or otoconia. Instead, their hair cells are covered by a gelatinous mass and respond to fluid motion at lower frequencies. They most likely do not respond to the method of stimulation used in this study since they don't resemble inertial accelerometers. Thus, the following interpretation on the directionality of afferents investigated in this study will assume that their input was received from hair cells of the saccule or the lagena.

Best directions for the vertical plane clustered around $90^{\circ}(75 \%$ of all directionally tuned afferents). These afferents did not respond to horizontal stimulation or only at very high levels. Such preference for $90^{\circ}$ angles in the vertical plane appeared to be correlated with the vertical orientation of the otolithic organs, the saccule and lagena, and the dorsoventral orientation of a large proportion of hair cells on these epithelia (Popper 1978, Lovell 2005). Some afferents responded to angles other than $90^{\circ}$ in the vertical plane. This spread may be explained by those hair 
cells of the lagena or saccule that are not dorsoventrally oriented (Fig. 3.1).

Considering the variety of hair cell orientations in the vertical plane, and especially of the lagena, one would however expect more afferents with BAs other than $90^{\circ}$ vertical. Instead, there is uniformity of afferent best responses to vertical plane stimuli at $90^{\circ}$, even though these afferents varied a lot in terms of thresholds and spontaneous activity. In $O$. tau, the best elevations could not always be predicted by hair cell orientation in the region innervated (Edds-Walton et al. 1999) and the curvature of the epithelia and interactions of the otoliths with the hair cell layer may result in unpredicted irregularites in directional tuning of afferents (Edds-Walton et al. 1999). The same may apply to afferent responses in A. fulvescens. During experiments, attempts were made to sample as completely and as uniformly as possible from saccular and lagenar afferents. The electrode was positioned at different locations of the posterior ramus of the eighth nerve for recordings, advanced through the entire nerve (in $1 \mu \mathrm{m}$ steps depth) and positioned on top of the nerve at multiple locations (at least about 10 different positions per experiment) to increase sampling variety.

The azimuths of primary afferents clustered around $0^{\circ}$. A subset of afferents $(8 / 42)$ showed a preference for angles between $-28^{\circ}$ and $-20^{\circ}$ which may be explained by the caudal part of the lagena being slightly angled to the side. However, the number of afferents showing best azimuths between $-28^{\circ}$ and $-20^{\circ}$ is small. Most afferents that responded to horizontal plane stimuli had BAs that did not deviate much from $0^{\circ}$, which can be explained by major areas of the saccule and lagena being oriented parallel to the midsaggital plane of the fish. All findings in teleost fishes studied so far indicated that the best azimuth of primary afferents innervating the 
saccule or lagena generally is in line with the orientation of the epithelium as seen in A. fulvescens (Fay 1984, Fay and Edds-Walton 1997a, Lu et al. 1998, Weeg et al. 2002, Lu et al. 2003, Meyer et al. 2004).

In teleost fishes, the saccule and lagena deviate from the midline of the fish at angles between $16^{\circ}$ and $40^{\circ}$ in azimuth (Fay 1984, Edds-Walton and Fay1997a, Lu et al. 1998, Lu et al. 2003). For any sound source, the stimulation of the right and left saccules (or lagenae) leads to differences in response magnitude arising from these epithelia in teleost fishes. This difference in response magnitude could then be used to centrally compute the azimuth of a sound source (first suggested by Sand 1974). This strategy would leave the animal with some ambiguities regarding certain azimuthal angles but ambiguities of this sort are faced by all two-eared animals, and could be compensated for by behavioral strategies using other senses (Fay 2005).

In A. fulvescens, a comparison between the overall output of the two ears may not be useful since most portions of the saccular and lagenar epithelia are oriented parallel to the midline of the fish and stimulation by a sound source located at the right or left would cause similar response magnitudes in both ears. One alternative mechanism to accomplish source location in azimuth would be through activation of hair cells on the horizontally oriented utricle. In C. auratus and D. latifrons, afferents innervating the utricle showed a wide range of BAs to horizontal plane stimuli (Fay 1984, Lu et al. 2004). This was consistent with the widespread morphological polarization of hair cells of the utricle in these species. The horizontally oriented utricle of A. fulvescens and a related sturgeon species (Scaphyrhinchus platorynchus) also has a wide range of hair cell orientations (Popper 1978, Lovell et al. 2005). Thus, 
it would be interesting to investigate utricular afferent responses to horizontal plane stimuli in A. fulvescens to test whether the utricle may play a role in encoding the particle motion axis in azimuth similar to C. auratus and D. latifrons. The use of interaural time differences would potentially be possible in adult $A$. fulvescens since adult animals have larger heads (assuming for instance a distance between the two ears of $10 \mathrm{~cm}$ would result in time differences of $65 \mu \mathrm{s})$.

Nine percent (16/177) of all directionally tuned afferents had DRPs with BAs at $90^{\circ}$ vertical and nearly $90^{\circ}$ in the horizontal plane. For an afferent with a best response at $90^{\circ}$ (vertical plane), one would normally expect to see a null in the horizontal plane if these afferents innervated hair cells that are oriented dorsoventrally. A response at $90^{\circ}$ in the horizontal plane may be explained by hair cells that are located at parts of the epithelia, which slightly bend into the horizontal plane (most caudal part of the lagena) or by hair cells that are tilted at parts of the epithelium that are uneven, thus their exact BAs could be best determined by moving the fish along axes in the frontal plane.

\section{Dependence of response strength $Z$ and phase angle on level and spontaneous activity}

According to the theory of vector detection, a population of directionally tuned afferents can resolve the major axis of particle motion but it does not determine which end of the axis pointed toward the source or away from it. This $180^{\circ}$ ambiguity could be solved if afferents encode the sound pressure and particle motion waveform separately (dual encoding). A phase shift of $180^{\circ}$ between the pressure and particle motion component of the waveform could be detected by two sets of afferents, which 
then could lead to central computations about the vector pointing to or away from the sound source ("phase-model," Schuijf 1975, Schuijf and Buwalda 1975). As a consequence, one would expect a bimodal distribution of phase angles in afferents, reflecting the variation in directional orientations of hair cells. Dual encoding is theoretically possible in species that can detect sound pressure through a swim bladder (or other gas filled structure) connected to the ear.

Phase angles however varied uniformly across afferents in A. fulvescens and did not simply fall into two groups, $180^{\circ}$ out of phase with one another. The same has been found in teleosts (Horner et al. 1981, Fay 1984, Fay and Edds-Walton 1997a, Weeg et al. 2002). In addition, for most units in A. fulvescens the phase angle changed with level (except for units with spontaneous activity larger than 70 spikes $\mathrm{s}^{-1}$ ). If considering the phase model as a way to solve the $180^{\circ}$ ambiguity, the results found in A. fulvescens would make the determination of the phase angle between particle motion and pressure rather difficult in this species. Thus, a $180^{\circ}$ ambiguity could potentially be solved through behavioral adjustments as in other vertebrates (e.g., humans) that face ambiguities such as sound source determination between the front and back of the head.

A correlation between phase angle and response magnitude (depending on stimulus level) for low spontaneous afferents leads to response phase or timing differences that could be used for intensity coding in A. fulvescens. For studies in teleosts it was proposed that an interaural response phase or timing difference between the saccules, for instance from the right and left ears of $O$. tau, played a role in representing response magnitude and thus giving rise to an interaural response 
timing code for azimuth (Fay and Edds-Walton 1997a). As mentioned before, a sound source stimulating the saccules and lagenae in A. fulvescens would not cause a strong difference in response magnitude between the two ears unless acoustic cues would play a role.

\section{Summary and evolutionary consequences}

A. fulvescens belongs to one of the few living representatives of a group of fishes that had their major radiation hundreds of million years ago in contrast to modern teleost fishes. This is the first study on physiological coding strategies (using single unit recordings) for direction in the auditory system of this group. For the interpretation of the results presented here, it is assumed as a first approximation that the coding strategies found in A. fulvescens, represent the ancestral condition of all fishes. It should be noted however, that during the course of its evolution, $A$. fulvescens might have developed characteristics in parallel to those of modern fishes. A full assessment of ancestral versus more modern coding strategies can only be made by comparing more species of the group of non-teleost Actinopterygian bony fishes (since it is obviously not possible to study physiological processes in fossils).

Afferents of A. fulvescens are very sensitive to the particle motion component of sound, some afferents showing phase-locked responses to displacements as low as $0.07 \mathrm{~nm}$ rms. This sensitivity is similar to that found in teleost fishes and other vertebrates, such as in the mammalian cochlea. Thus, the presence of otoconia as well as the fact that juvenile $A$. fulvescens were used in this study apparently did not result in unusually poor sensitivity of the ear. The mechanisms responsible for such low 
sensitivities may have appeared first in non-teleost Actinopterygian fishes and has been retained throughout the evolution of vertebrates.

Many physiological characteristics of the auditory periphery of A. fulvescens were very similar to those in teleosts including the spontaneous rates, wide variety of spontaneous rates and thresholds, varying phase angles, and cosine-like DRPs. These common physiological characteristics may reflect important functions that have been conserved throughout the evolution of fishes or (for some functions) of all vertebrates. An alternative way to interpret these results found in A. fulvescens is that the process of convergence resulted in similar coding strategies for sound source location in phylogenetically unrelated species. This would indicate the possibility that selective pressures lead to only a few coding strategies that an aquatic auditory system can evolve. However, it seems likely that similiarities occurred because the auditory system in vertebrates derived from a common ancestor. The assumption of common ancestry is based on the notion that certain auditory/vestibular structures are considered homologous, such as the vertebrate hair cell (Coffin et al. 2004) and on the observation that the gross morphology of the inner ears is very similar among vertebrates.

The first evidence for hearing and sound sensitivity to sound of the inner ear was found in elasmobranchs (Loewenstein and Roberts 1950, Myrberg et al. 1972, Nelson and Johnson 1976, Corwin 1981). Elasmobranchs have three otolith organs with the lagena and saccule located in one pouch with vertical orientation in space (just as in A. fulvescens) and a horizontally oriented utricle. Therefore it seems plausible that elasmobranchs most likely show similar coding strategies for direction 
to ancestral bony fish and teleosts. The significance of hearing in Agnatha still remains to be investigated.

Mechanisms for sound source location in A. fulvescens

The cosine-like directional tuning found in horizontal plane as well as vertical plane DRPs of afferents can serve as the basis for coding the location of a sound source in A. fulvescens. The great majority of afferents have directional properties (cosine-like) that would be expected if they innervated a single hair cell or a group of hair cells having the same directional orientation.

In general, best axes in the horizontal plane tended to lie parallel to the orientation of the saccule and lagena in the horizontal plane, which means along the midline of A. fulvescens. Therefore best axes of the major areas of the saccules and lagenae are also parallel to each other and therefore cannot give rise to interaural differences in response magnitude between the two ears. One potential mechanism to compute sound source azimuth in A. fulvescens would be by the help of the utricle.

Since afferents in A. fulvescens seem to have the capacity to determine the axis of acoustic particle motion, a computation of the direction of a sound source in the vertical is possible although limited in A. fulvescens due to the preference for $90^{\circ}$ in the vertical plane. Other computational strategies may be used, for instance, a comparison of the inputs from afferents innervating the horizontally oriented utricle with the inputs from vertically oriented saccule and lagena to compute the location of a sound in azimuth and elevation centrally. Non-auditory strategies (body movements) may be used to compensate the limitations found in A. fulvescens. 
Finally, despite the difference in otolith structure (otoconia versus otolith in teleosts), the phylogenetic position of the family Acipenseridae (occurring much earlier in vertebrate evolution than teleosts) and the fact that juvenile A. fulvescens had to be used in this study due to size limitations of the experimental setup and availability, responses from eighth nerve afferents show striking similarities to experiments conducted in adult teleost fishes using the same stimulation and recording method. 

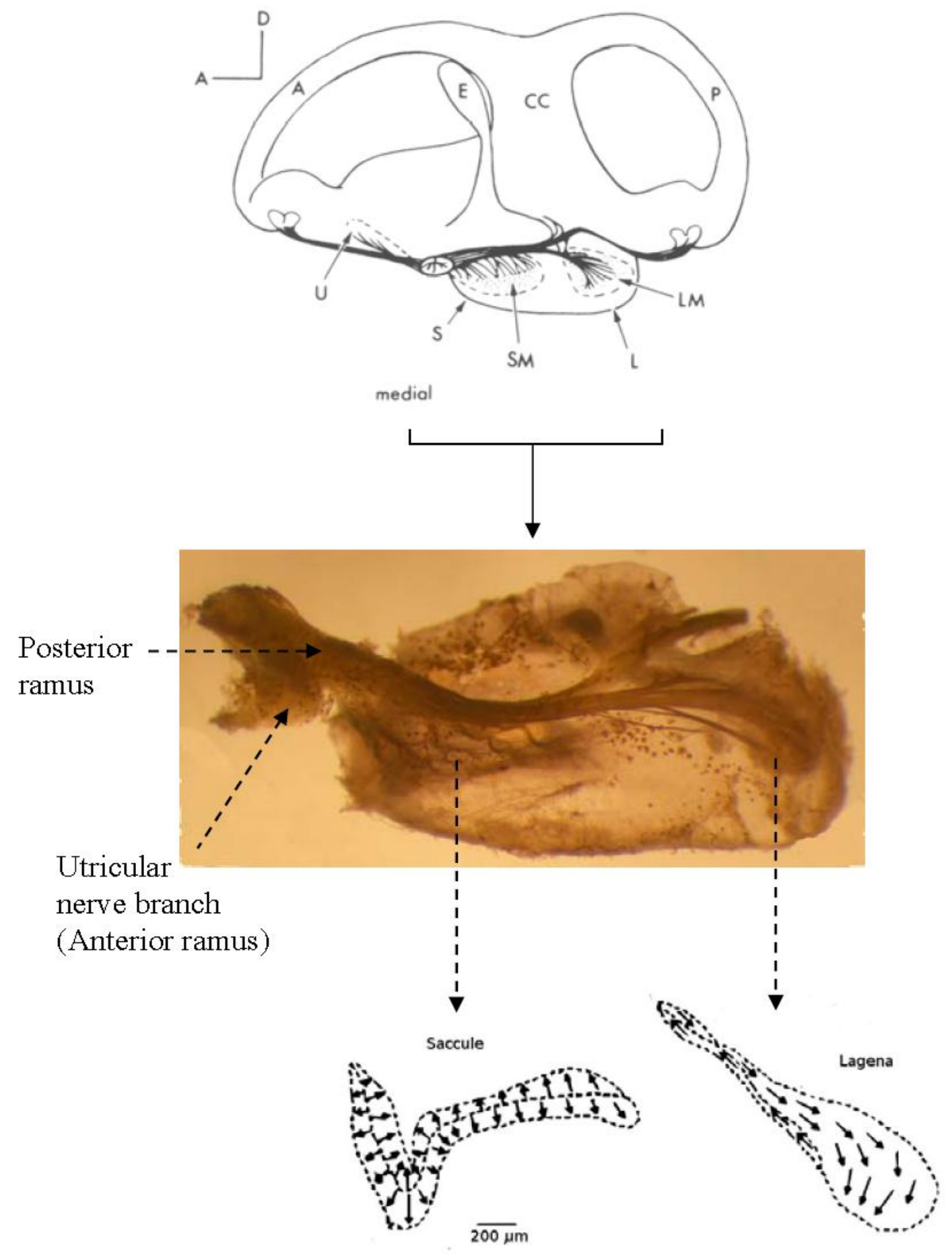
Fig. 3.1: Top: right ear and innervation of Acipenser sturio (Retzius 1881). Abbreviations: A - anterior semicircular canal, CC - Crus commune, E Endolymphatic duct, L - Lagena, LM - Lagenar macula (epithelium), P - Posterior semicircular canal, S - Saccule, SM - Saccular macula, U - Utricle. Middle: innervation of the right saccule and lagena in A. fulvescens (photograph taken by M.Meyer). Bottom: hair cell orientation pattern in A. fulvescens (adapted from Lovell et al. 2005). 


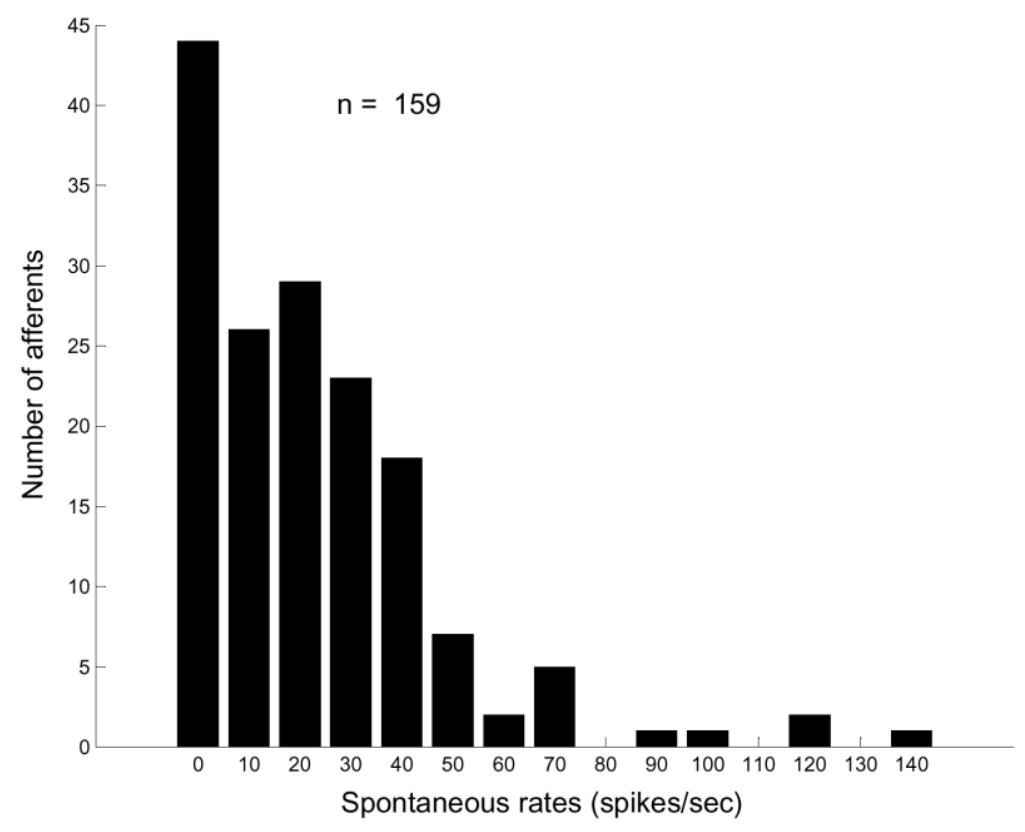

Fig. 3.2: Distribution of spontaneous rates for 159 afferents. Bins are 10 spikes s $^{-1}$ except for first bin $\left(5\right.$ spikes s ${ }^{-1}$ : range $=0$ to $\left.\leq 4.9\right)$. 


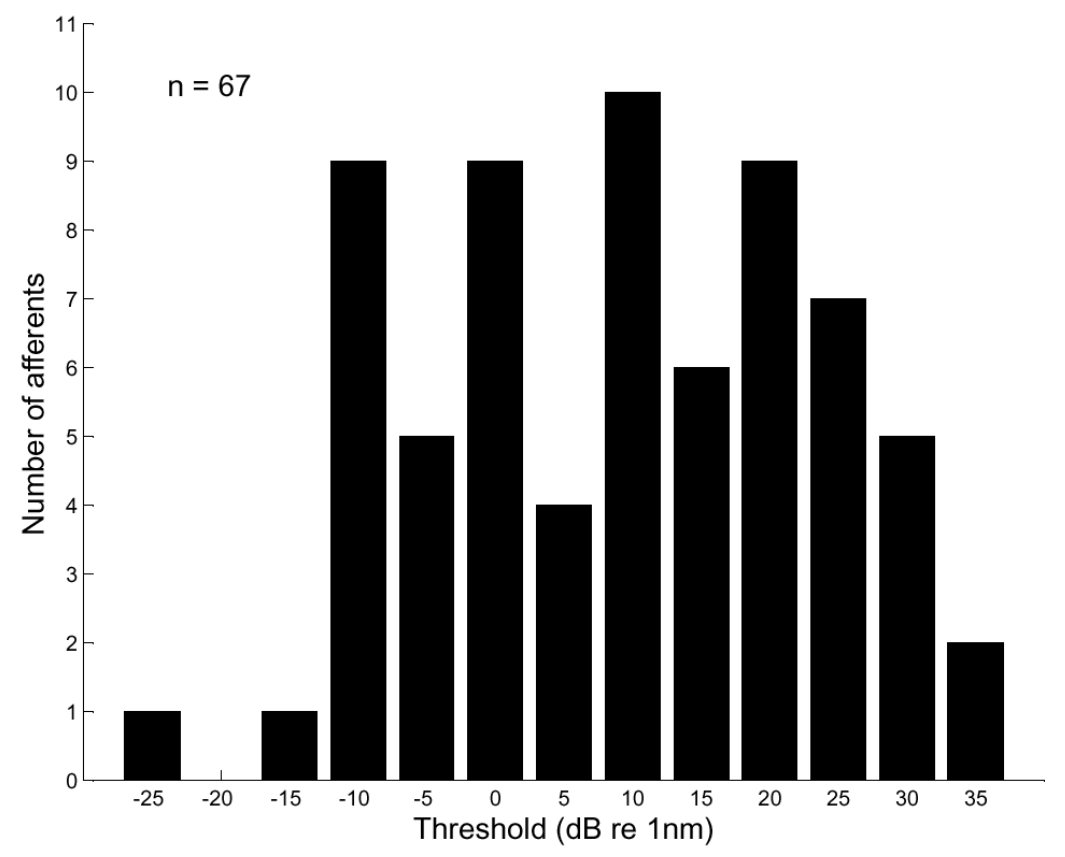

Fig. 3.3: Distribution of absolute displacement thresholds $(Z \leq 20)$ in response to 100 $\mathrm{Hz}$ at $90^{\circ}$ vertical and $0^{\circ}$ horizontal stimulation for 67 afferents. Bins are $5 \mathrm{~dB}$. 


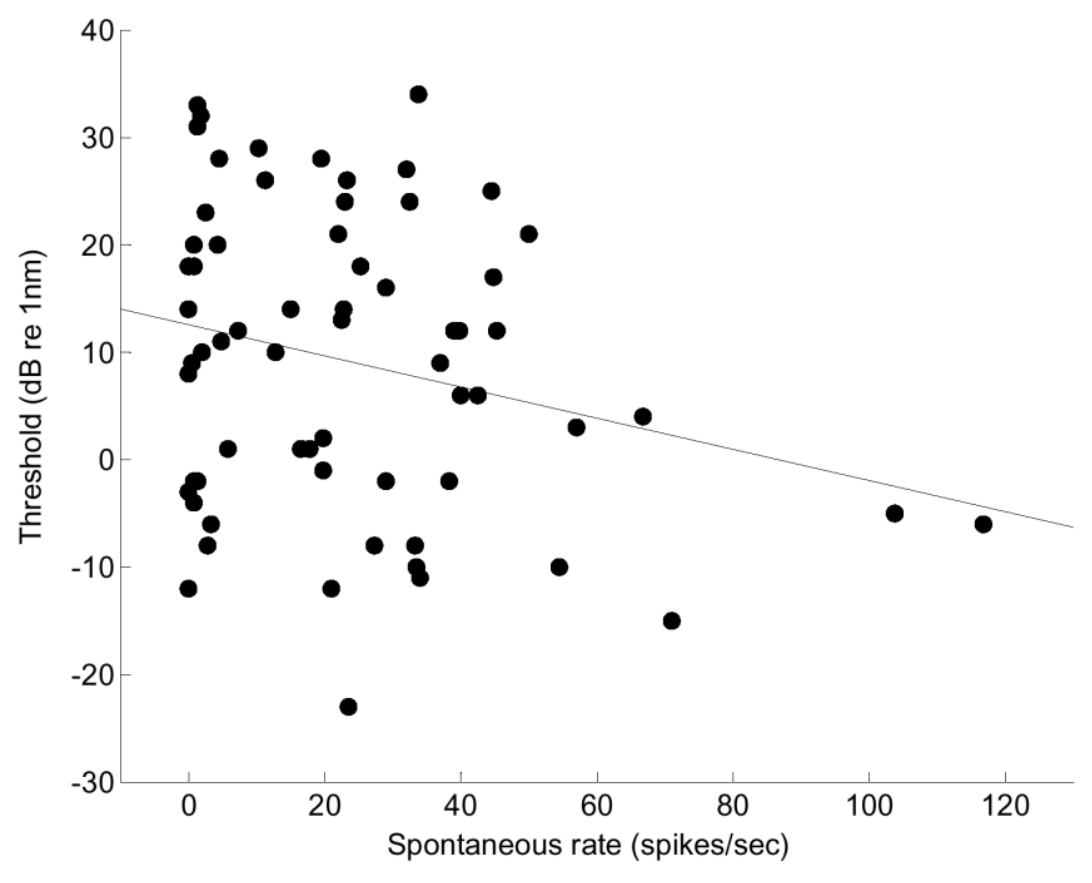

Fig. 3.4: Significant correlation between spontaneous rate and threshold as determined for 65 afferents $(\mathrm{r}=-0.25, \mathrm{n}=65, \mathrm{p}<0.05$, two tails $)$ but without outliers (two data points with highest spontaneous rates) the relationship is not significant ( $\mathrm{r}=$ $-0.17, \mathrm{n}=63)$. 
pp11

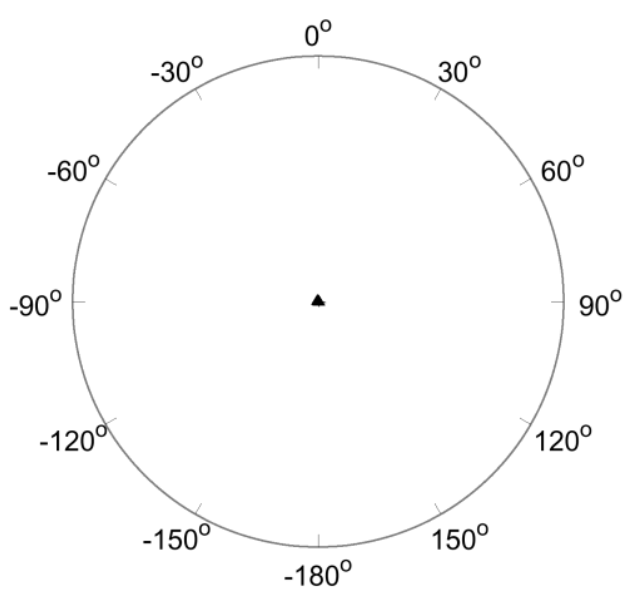

oo2

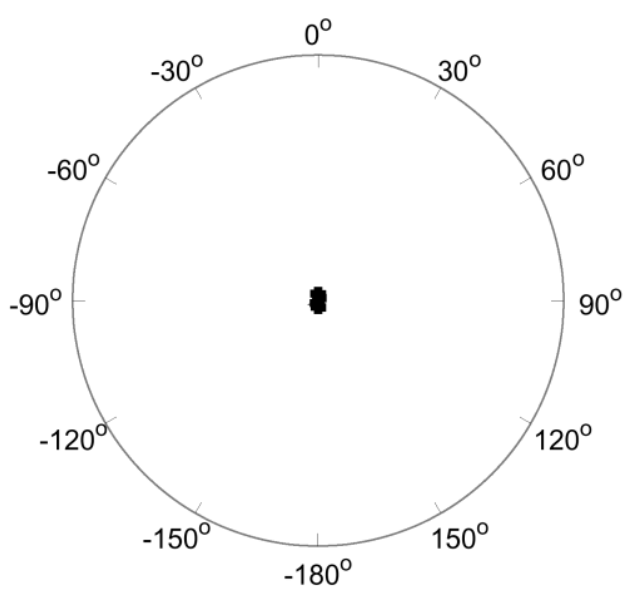

bb13

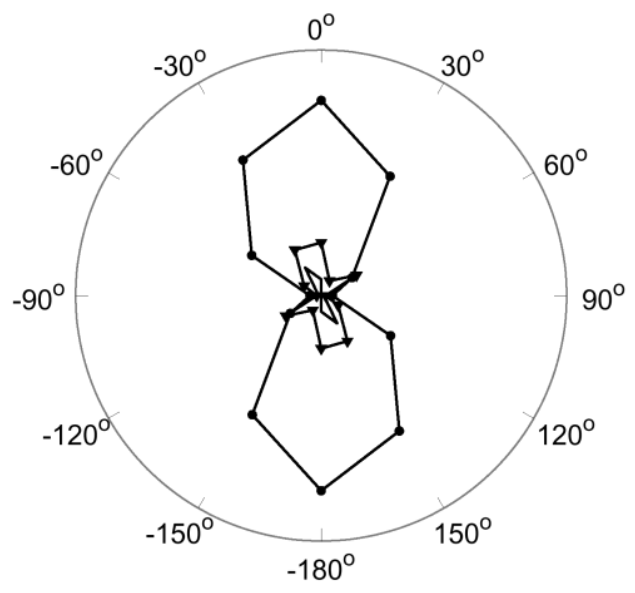

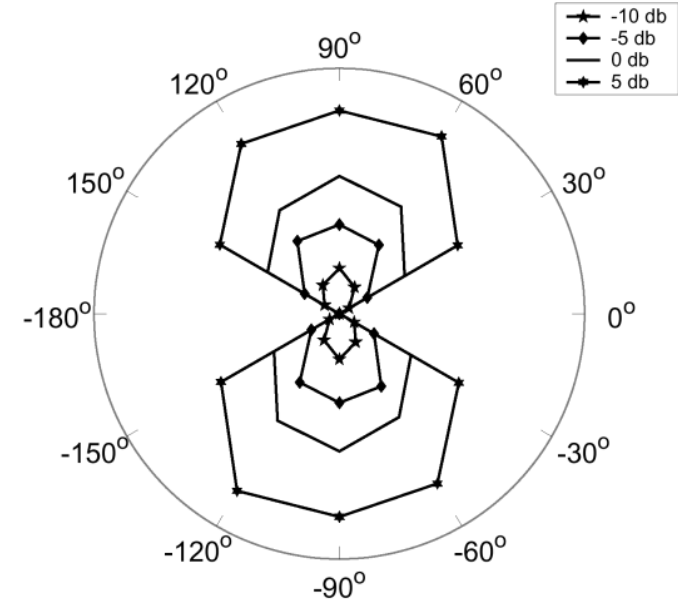
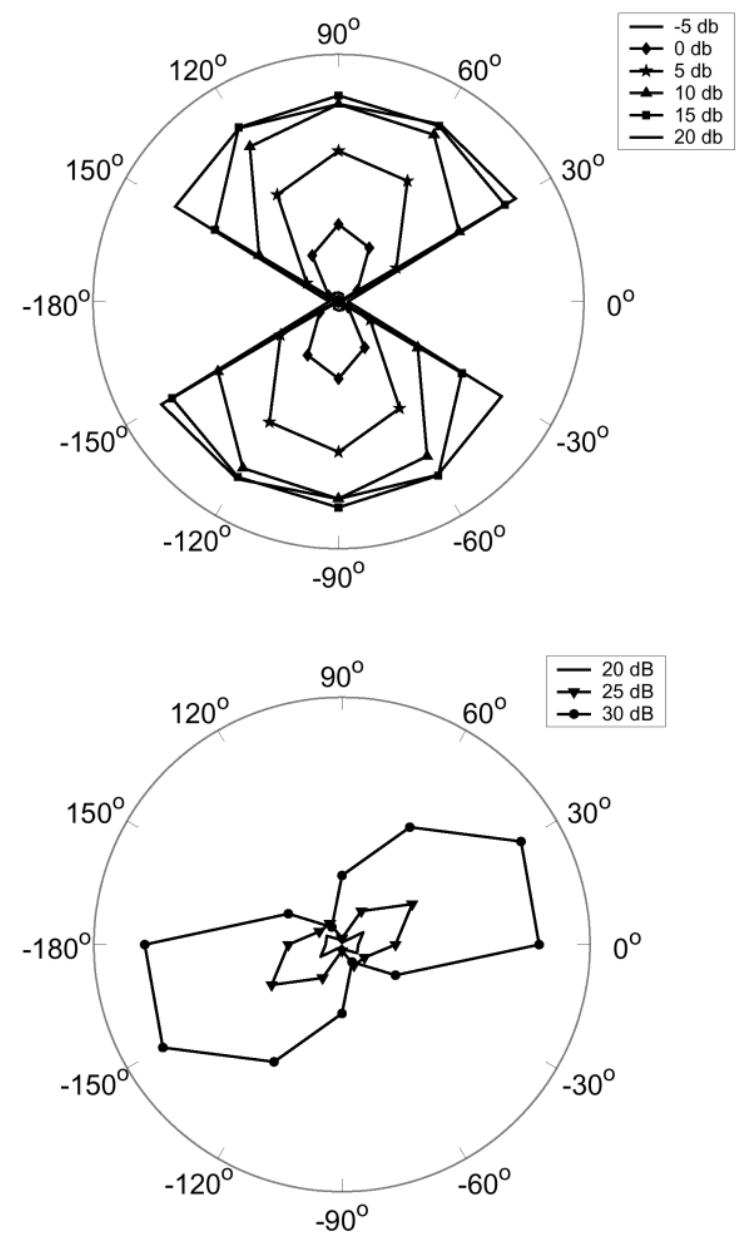
ff11
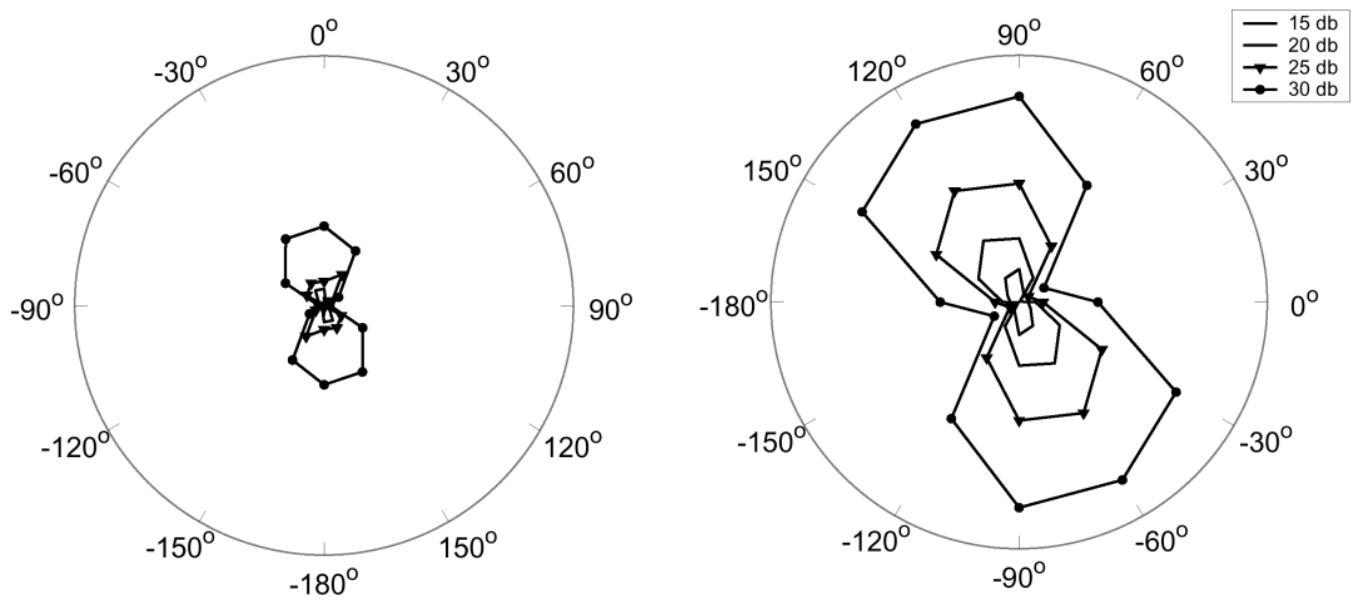

$\mathrm{mm} 14$
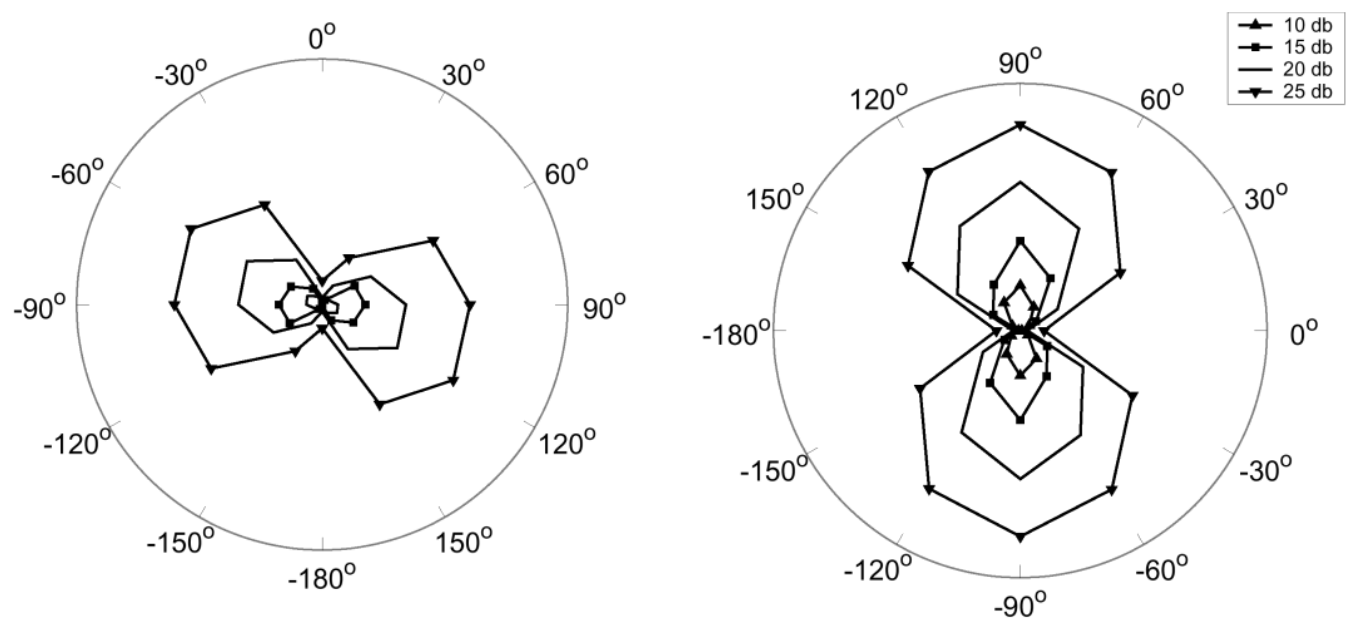

Fig. 3.5: DRPs in the horizontal (left column) and vertical (right column) planes for five representative afferents obtained at various levels (see legend to the right for each pair). The maximum amplitude (max. response) for each unit was: pp11: $Z=304$, oo2: $Z=342$, bb13: $Z=42$, ff11: $Z=278, \mathrm{~mm} 14: Z=243$. 

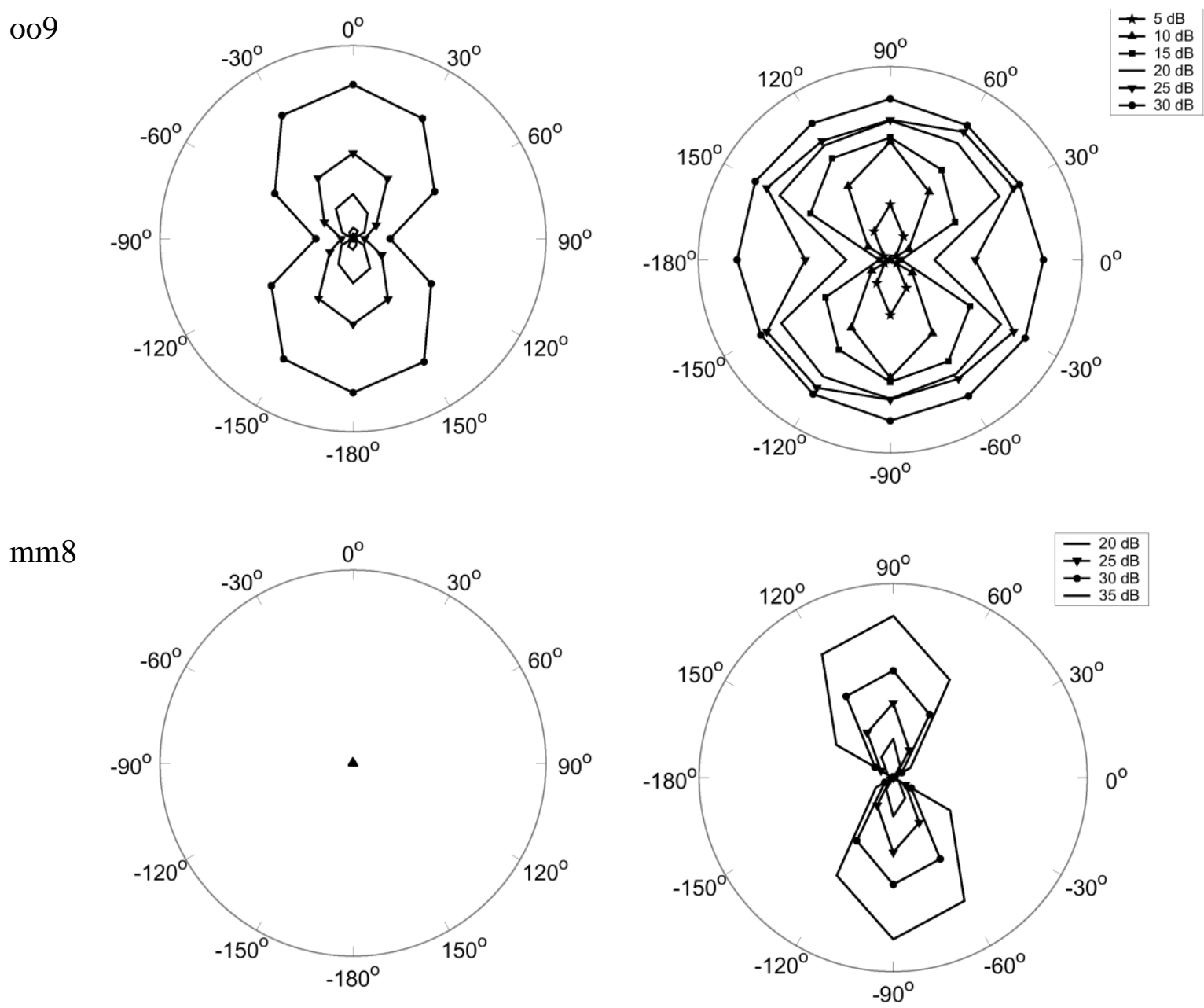

Fig. 3.6: Two afferents that represented extreme cases in terms of DRP shape. See text for details. The maximum amplitude (max. response) for each unit was: oo9: $\mathrm{Z}=415, \mathrm{~mm} 8: \mathrm{Z}$ $=189$. 


\section{Horizontal}

ff1

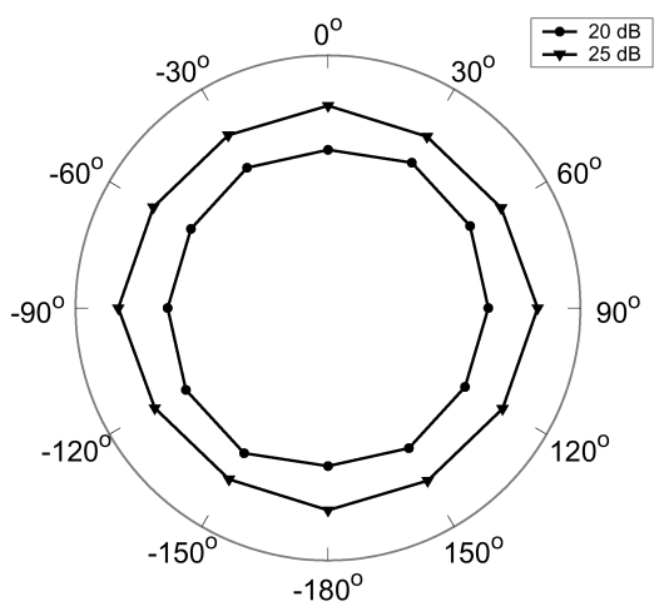

Vertical

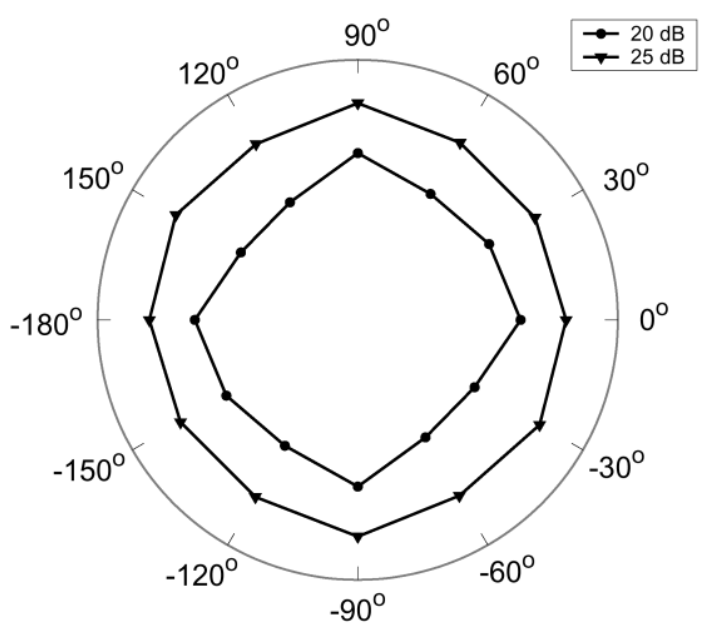

Fig. 3.7: Example of a non-directional unit with Z-values lower than 5. Spike rate is plotted as a function of stimulus angle. Spike rate increased with level. Units with this response pattern have been classified as efferents in teleosts (Edds-Walton et al. 1999, Weeg et al. 2002). Average spike rates: 55 spikes s $^{-1}(20 \mathrm{~dB}), 68.9$ spikes s$^{-1}(25 \mathrm{~dB})$, background rate: 26 spikes $\mathrm{s}^{-1}$. 

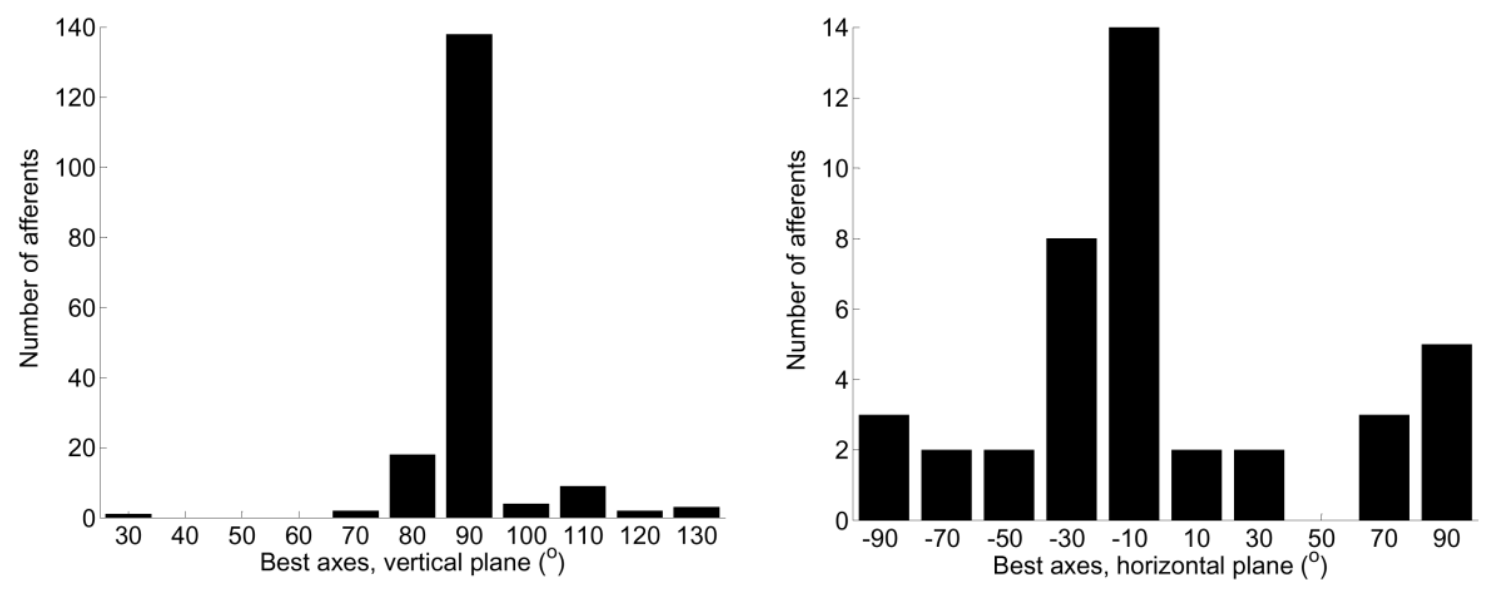

Fig. 3.8: Distributions of BAs in the vertical (left, $n=177$, binwidth: $10^{\circ}$ ) and horizontal plane (right, $\mathrm{n}=42$, binwidth: $\pm 20^{\circ}$ ). Note: 135 afferents did not show any response to stimuli in the horizontal plane. 
A

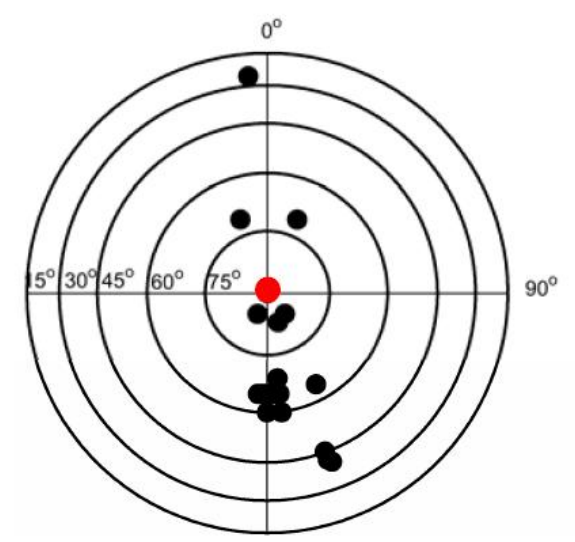

$B$
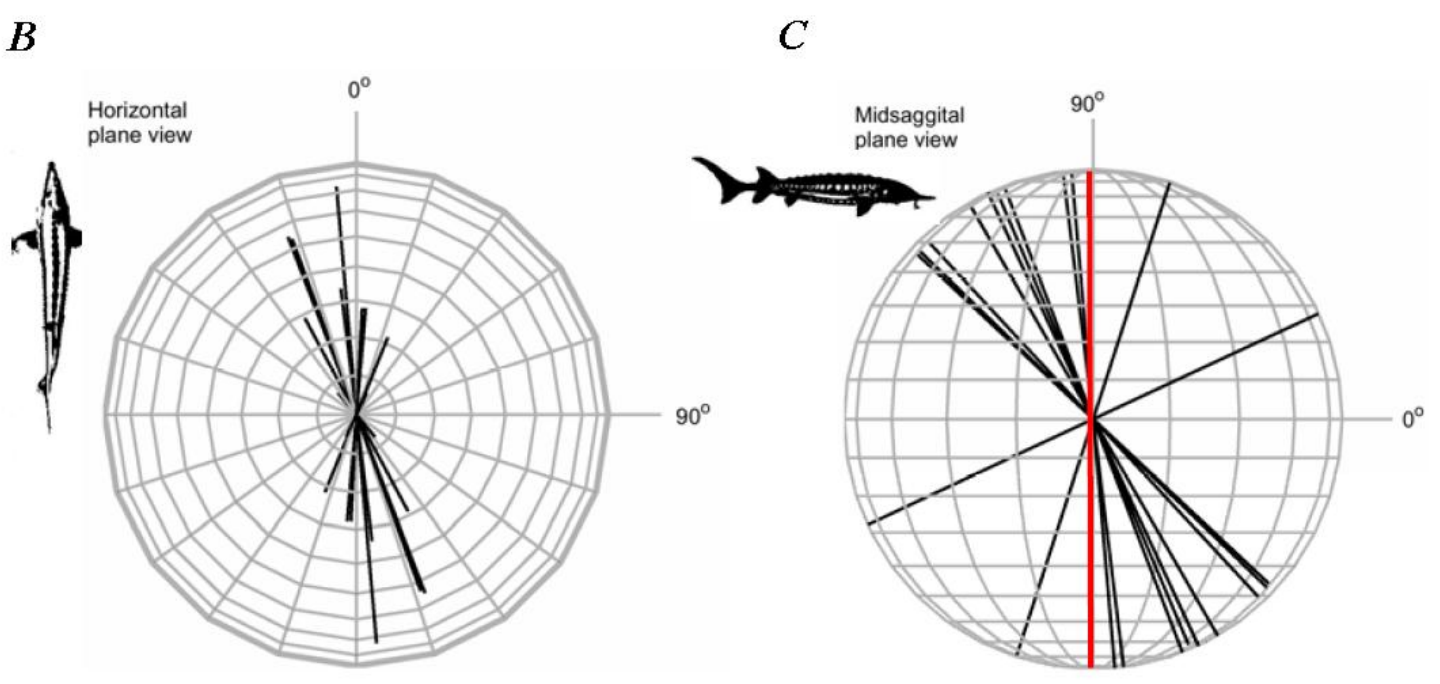

Fig. 3.9: Best axis in three-dimensional space for 161 afferents plotted at different perspectives. A: Top view of a globe with the fish at the center. Concentric circles represent elevations (cross point at the center represents $90^{\circ}$, red dot; note that most afferents have their best axis at this point). Each point represents the location on the globe's surface at which one afferent's best axis penetrates the surface. $0^{\circ}$ azimuth is in front of the fish; $90^{\circ}$ azimuth is to the right of the fish. B: horizontal plane projection of the $3 \mathrm{D}$ vectors. C: vertical plane projection of the $3 \mathrm{D}$ vectors. Note: most fibers (135) only responded to $90^{\circ}$ stimuli in the vertical and not to horizontal stimulation as illustrated by the red dot in $\mathbf{A}$ and the red vector in $\mathbf{C}$. 
A

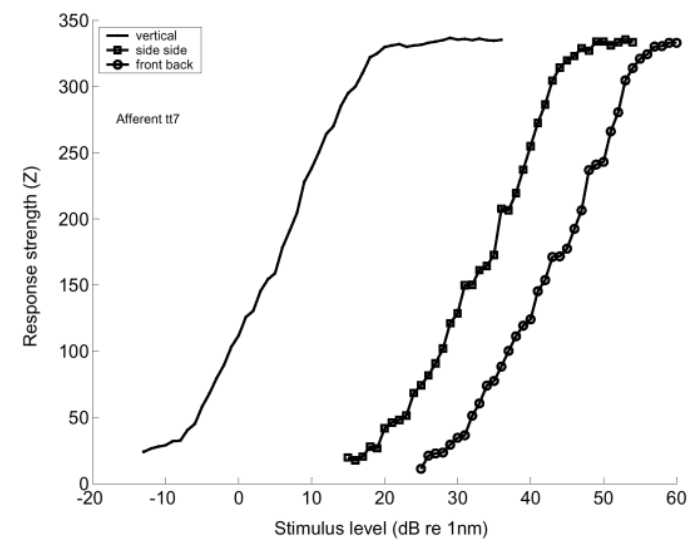

B

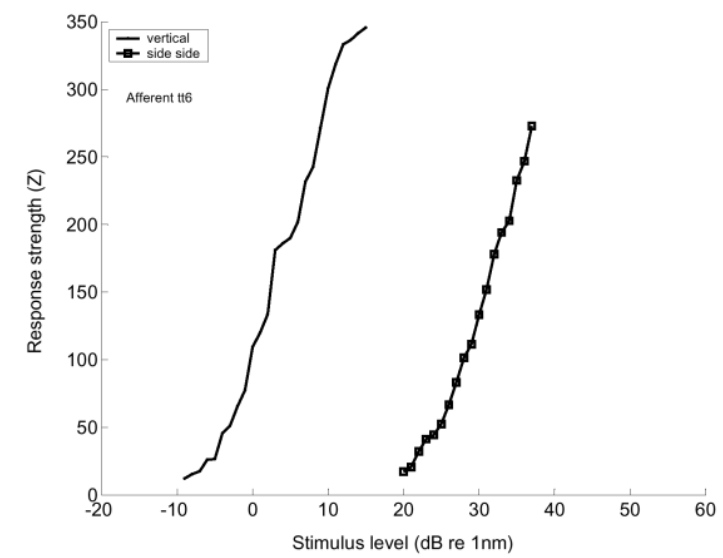

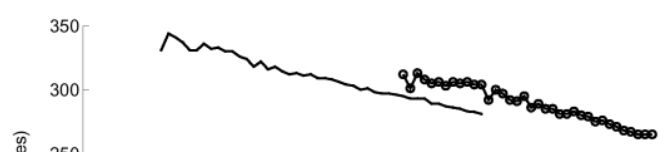

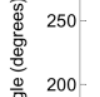

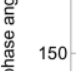

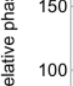

50
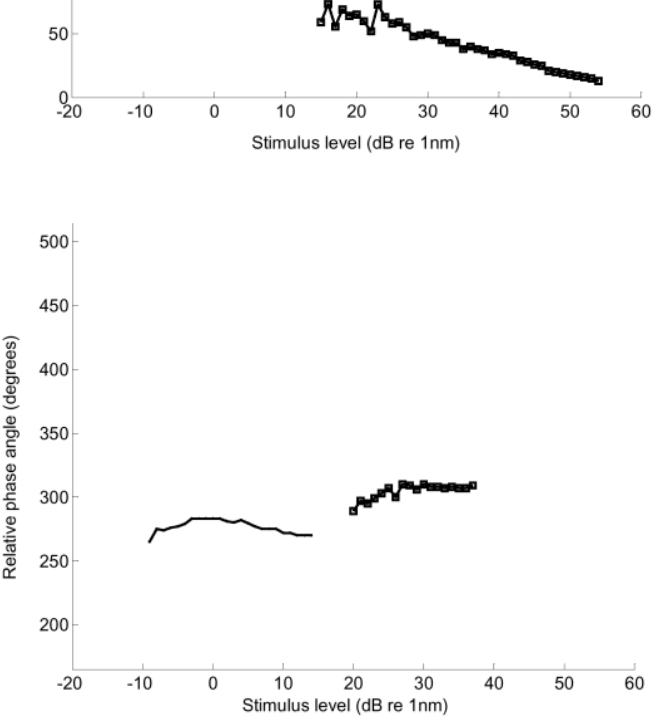

Fig. 3.10: Response versus level functions and phase angle versus level functions for two representative afferents with different spontaneous activity. Legends on the left of each pair indicate functions obtained at vertical, side-side, or front back stimulation (when available). A: unit tt7, background activity: 54.5 spikes $\mathrm{s}^{-1}$. B: unit tt6, background activity: 117 spikes s$^{-1}$. Data for front back stimulation are not available for tt6. 
CHAPTER IV

\section{A mathematical model to quantify directional and non- directional response profiles in Acipenser fulvescens, the lake sturgeon}

\section{Introduction}

In Acipenser fulvescens (the lake sturgeon) most directionally selective afferents innervating the saccule and lagena showed a directional response profile (DRP) in the horizontal and vertical planes that approximately resembled a cosine function (Chapter 3). This is consistent with DRP data from teleosts and matches the directional tuning of an individual hair cell (Hudspeth and Corey 1977). In some cases, however, the profiles differed sufficiently from a cosine shape to suggest deeper investigation of the DRP shape. Some DRPs looked flattened and broadened as compared to a cosine function whereas others appeared narrow. Such deviations of DRPs from a cosine-shape have also been described qualitatively in the past for teleosts (Fay and Edds-Walton 1997a, Weeg et al. 2002, Lu et al. 2003, 2004) but the nature and extent of the deviations have not been quantified. This study seeks to quantitatively fit the data obtained in A. fulvescens with a mathematical model that can serve as a description of the measured DRPs. Such a mathematical description is useful, for example, in generating computational models of central auditory coding, or for creating formal hypotheses of how hair cell responses are integrated by afferents (Fay and Edds-Walton 1997a, Fay and Edds-Walton 1999, Fay and Edds- 
Walton 2001, Ma and Fay 2002, Edds-Walton and Fay 2003, Edds-Walton and Fay 2005).

The mathematical model used here to model the observed DRP-shapes is a modified cosine function. This function has only one more parameter than the cosine function, but it captures the observed distortions in the DRP-shape including flattening and narrowing as well as the omni-directionality measured in a few cells characterized as efferents (Chapter 3). Fitting the observed DRP data to the modified cosine function results in a quantitative description of DRP-shape in the form of model parameter values. These numerical values are used to investigate the effects of certain stimulus parameters, such as stimulus level, on DRP-shape, which is a second goal of this study.

\section{Methods}

Data used in this study were obtained from previous recordings of saccular and lagenar afferents in the A. fulvescens (for methods and results, see chapter 3). Data from 74 afferents were fitted using the model and used to investigate the effects of level on DRP-shape.

Most units used in this study were directionally selective (70/74) and a few examples were not (4/74), which means that they did not have a cosine-like DRP and instead showed an omni-directional response profile. For the directionally selective afferents, the Z-value (as a measure of response strength) was plotted as a function of stimulus angle. $\mathrm{Z}$ was defined as: $\mathrm{Z}=\mathrm{N}_{\mathrm{s}} \mathrm{R}^{2}$, where $\mathrm{N}_{\mathrm{s}}$ defines the number of spikes and $\mathrm{R}$ represents the coefficient of synchronization (chapter 3 ). For non-directional 
afferents, instead of $Z$, the spike rate was plotted as a function of stimulus angle (since these units were not phase-coupled to the stimulus waveform). Only the response profiles obtained from stimulation in the vertical plane were chosen since many afferents did not respond to horizontal plane-stimuli (Chapter 3). However response profiles obtained for stimuli in the horizontal plane had similar shapes to data obtained in the vertical plane (Chapter 3). For each afferent (used in this investigation), response profiles were obtained at different stimulus levels mostly within the unit's dynamic range.

The formula used to fit the response profiles was:

$$
\left.Z=Z_{\text {max }} \mid \cos \theta-\alpha\right]^{k}
$$

Here $\mathrm{Z}$ = the fitted $\mathrm{Z}$ value (or $\mathrm{Z}$-value of the model-response), $\mathrm{Z}_{\max }=$ the $\operatorname{maximum} \mathrm{Z}$ value or $\mathrm{Z}$ value at best axis (BA) for a given level, $\theta=$ any angle tested, $\alpha=$ the angle of the BA. When $\theta=\alpha$, then $\mathrm{Z}=\mathrm{Z}_{\max } . \mathrm{K}$ can be called "distortion factor". When $\mathrm{K}=1$, the fit is an exact cosine-function (Fig. 1A) and there is no distortion. For $\mathrm{K}>1$ the cosine functions becomes "narrow" (Fig. 1B), and for $\mathrm{K}<1$, the cosine function becomes "flattened" (Fig. 4.1C).

For each afferent two fits were performed. In one $\mathrm{K}$ was forced to be one $(\mathrm{K}=$ 1), which resulted in a pure cosine fit. In the other, $\mathrm{K}$ was optimized along with the other parameters resulting in a modified cosine fit. For both fits, an error for each DRP at any given level was computed and plotted as a function of stimulus intensity. The error was computed using the following formula:

$$
\left.\mathrm{E}(\text { level })=\frac{1}{N} \sum_{n=1}^{N} \mid \frac{Z_{n}^{\prime}-Z_{n}}{\max \boldsymbol{C}_{n}}\right\rfloor
$$


Here (for each DRP): $N=$ total number of data points, $\mathrm{Z}_{\mathrm{n}}{ }^{\prime}=$ fitted $\mathrm{Z}$ - value, $\mathrm{Z}_{\mathrm{n}}=$ experimental $\mathrm{Z}$-value, $\max \left(\mathrm{Z}_{\mathrm{n}}\right)=$ maximum $\mathrm{Z}$ value of all experimental $\mathrm{Z}$ values at one particular level (corresponding to the $\mathrm{Z}$ value at, or near, the $\mathrm{BA}$ ).

To test the effect of level on DRP-shape, the change of the distortion factor K was investigated with level.

\section{Results}

The three examples in Figures 4.2, 4.3, and 4.4 each show the experimental DRP data, the model fits, the variation of the model parameter value $\mathrm{K}$, and the error with different stimulus parameters, for three different units. In each figure, panel A shows the unit's measured DRP overlaid with the best cosine fit $(K=1$, all other parameters optimized for best fit) for each level. Panel B shows the unit's measured DRP overlaid with the best modified cosine fit (all parameters optimized for best fit). Panel C and D show model parameter variation with stimulus intensity while panel E shows variation in model fit error, also with stimulus intensity.

Units have been identified with letters and numbers, where letters (aa, bb, cc) were used to designate specific animals, and numbers to indicated a specific unit in that animal (e.g., " $t \mathrm{t} 7$ ” stands for unit number seven, recorded from animal tt). Units differed mainly in how the K-value changed with level. For unit pp18, the K-value for the modified cosine fit was larger than one at stimulus-levels near the threshold of the unit (please compare Figs. 4.2 C with D), reached values around one within the unit's dynamic range, and exceeded one when the unit reached saturation. 
The values for $\mathrm{K}$ (being 1, larger or smaller than 1) reflected the shape of the DRP, which changed with level. At levels near the threshold of the unit, the DRPshape was narrow, within the unit's dynamic range, the unit looked more like a perfect cosine, and at saturation points, the DRP-shape appeared flattened. The error values for the modified cosine model were generally smaller than the error values for the cosine model indicating a better fit. The $\mathrm{U}$ shape of the error curve for the cosine fit indicates that only at the intermediate intensity levels (corresponding to the linear region of the response-level curve) was the cosine a good fit to the data. At higher and lower stimulus intensities the distortion in the DRP (from a cosine fit) is captured by the modified cosine model. This distortion effect can also be seen in panel D where $\mathrm{K}$ takes on values close to one only in the middle of the intensity range.

The second example (unit tt7) differed from unit pp18 in terms of how the Kvalue (for each DRP) changed with level. On first glance (without quantification), the DRPs of the two units pp18 and tt7 looked very similar. However, the K-values in unit tt7 were smaller than one (also see Figs. $4.3 \mathrm{C}$ and D) at all levels at which the unit was tested. Interestingly, $\mathrm{K}$ was smaller than one even within the unit's dynamic range (levels 5 to $15 \mathrm{~dB}$; levels near threshold of the unit are not available) fitting to the more flattened shape of the unit. The modified cosine fit seemed to be the best fit for most DRPs (obtained at different levels) of this unit.

Unit ff1 was not directional resulting in omni-directional response profiles (Figs. 4.4 A or B). For this unit, level did not have any effect on the response profile shape or size (Fig. 4.4 C). Consequently, the K-values and the error values remained constant with level (Figs. 4.4 D, E). K reached values near zero to fit this response 
profile. The modified cosine function was a good fit for the data resulting in very low error values (and a lot lower than the cosine-fit).

The maximum response strength $(\mathrm{Z})$ was obtained from DRPs fitted by the modified cosine model and plotted as a function of stimulus level (Fig. 4.5) for the 70 directionally selective afferents. As can be obtained from the graph, the dynamic range, slopes, and the spread in Z-values were similar between units but afferents varied widely in threshold. The consistency in the spread of Z-values was useful for the interpretation of the following two graphs.

Figure 4.6 compares the mean errors for both models as a function of the maximum $\mathrm{Z}$ value (of each DRP). Since the spread in $\mathrm{Z}$-values was consistent with level among most units (Fig. 4.5), a high, medium, or low Z-value would reflect whether the unit is stimulated at high, medium, or low levels even if the stimulus level for that particular unit is not specified.

On average the modified cosine fit resulted in smaller error values at all Zvalues (and levels) than the cosine fit. The U-shape of the cosine-fit indicated that this model resulted in high error-values at very low and very high Z-values. The modified cosine fit worked well at low and especially high Z-values.

Figure 4.7 investigates the effect of Z-value (or level) on the K-value of the modified cosine fit. There was a significant correlation between the two parameters $(\mathrm{R}=-0.62, \mathrm{~N}=294, \mathrm{p}=0.05$, two-tailed) when plotting the $\mathrm{K}$-values on a logarithmic scale. On a linear scale the K-value decreased exponentially with increasing response strength (or level). However, K-values smaller than one (flattened DRPs) also occurred at low $Z_{\max }$-values (levels) indicative of units that had a flattened 
DRP-shape at a wide range of levels (also within their dynamic range). In addition, $\mathrm{K}$-values larger than one (narrow DRPs) also occurred at high $\mathrm{Z}_{\max }$-values (levels), which can be explained by units that maintained a narrow DRP-shape even at higher levels. Table 4.1 shows the distributions of units that were categorized based on how K changed with level. For 37\% of the units most of their K-values were larger than one within their dynamic range (for most of those units, the level of saturation however was not known). For $31 \%$ of the units $\mathrm{K}$ changed from values smaller than one to values larger than one indicating a more narrow shape near threshold and a more flattened DRP-shape at saturation (e.g., pp18). Twenty-one percent of the units had K-values smaller than one at most levels within their dynamic range (e.g., tt7). Only $2 \%$ of the units had K-values corresponding to one at all levels except the level near threshold.

\section{Discussion}

The modified cosine fit was the better fit (leading to smaller error values) for all response profiles (whether they were obtained near threshold, within the dynamic range, or at saturation of the unit) of all afferents, since it adjusted to variations from the cosine fit including a more narrow, flattened, or omni-directional shape.

The value of $\mathrm{K}$ was used to investigate the effect of stimulus level on the DRP-shape of units. Taking together the K-values from all DRP-data, a correlation between $\mathrm{K}$-value and Z-value (that was proportional to the levels individual units were tested at for most units) was found (Fig. 4.7). With increasing Z-value or level, the K-value decreased exponentially. Thus, an increase in stimulus level caused most 
DRPs to become less narrow (e.g., unit pp18, Fig. 4.2). Near threshold, $\mathrm{K}$ was always larger than one for all units (for which those data were available; e.g., pp18, Fig. 4.2).

In most studies on teleosts, narrowing of DRP-shape was interpreted as a threshold effect (occurring at stimulus levels near the threshold of the unit) and flattening as a saturation effect (due to the unit going into saturation at higher levels) (Weeg et al. 2002, Ma and Fay 2002, Lu et al. 2003). However, such a change of DRP-shape with level was only seen in some units, maximally in $68 \%$ of the units investigated in this study including those units for which the level of saturation was not known. In contrast, not all DRPs were purely cosine shaped within their dynamic range (which was linear for all directional afferents, Fig. 4.5), such as seen in unit pp18 (Fig. 4.2). In fact, for $30 \%$ of the units, $\mathrm{K}$ was smaller at many levels within the unit's dynamic range (e.g., tt7, Fig. 4.3; table 4.1). Thus, other processes besides level might lead to deviations from a pure cosine-shape (which is the shape seen for single hair cells, in the auditory periphery of fish, Hudspeth and Corey 1977). The deviations from the cosine towards a more flattened shape may be the result of single afferents innervating hair cells varying slightly in hair cell orientation. Units with Kvalues less than one would then be likely to receive inputs from hair cells with a slight diversity of best axes. In the extreme case where the unit receives equal input from hair cells in-all directions, the K-value would be near zero. Conversely, those afferents having K-values close to one would reflect input from hair cells with the same orientations.

Studies in teleost fishes have shown that afferents innervate numerous hair cells in one otolith organ (Presson et al. 1992, Edds-Walton and Popper 1995, Edds- 
Walton et al. 1999). Indeed, studies in Opsanus tau (Oyster toadfish) using intracellular injections of neurobiotin showed that a single saccular afferent had up to 111 synaptic sites (Edds-Walton et al. 1999), yet those afferents would still be very directional (showing cosine like DRPs). Variations in DRP-shape, such as seen for $A$. fulvescens, were also observed in $O$. tau. For future experiments, anatomical studies in A. fulvescens studying the correlation of directional selectivity with the hair cell orientation pattern (including the number of hair cells innervated) would be useful to verify that a flattened DRP-shape can be caused by one afferent innervating hair cells with slightly different orientations. Lu et al. 2004 also found that some utricular afferents showed flattened and narrow shapes within the unit's dynamic range.

In summary, the modified cosine fit was the better model to describe the variations of DRP-shape in the auditory periphery of A. fulvescens and the distortion factor K captured all possible variations described in Chapter 3 (corresponding to the variations of response profiles seen in teleosts). Therefore, the K-value can be used to describe the effect on DRP-shape of certain parameters. Thus, for future modeling experiments (e.g., describing and quantifying central computations), the modified cosine fit may be a better description than the plain cosine function.

Altogether, this model allows to quantitatively describe directional properties in the auditory periphery of fish, which gives us a better understanding of the periphery's contribution (at the hair cell level) to sound source location mechanisms. 

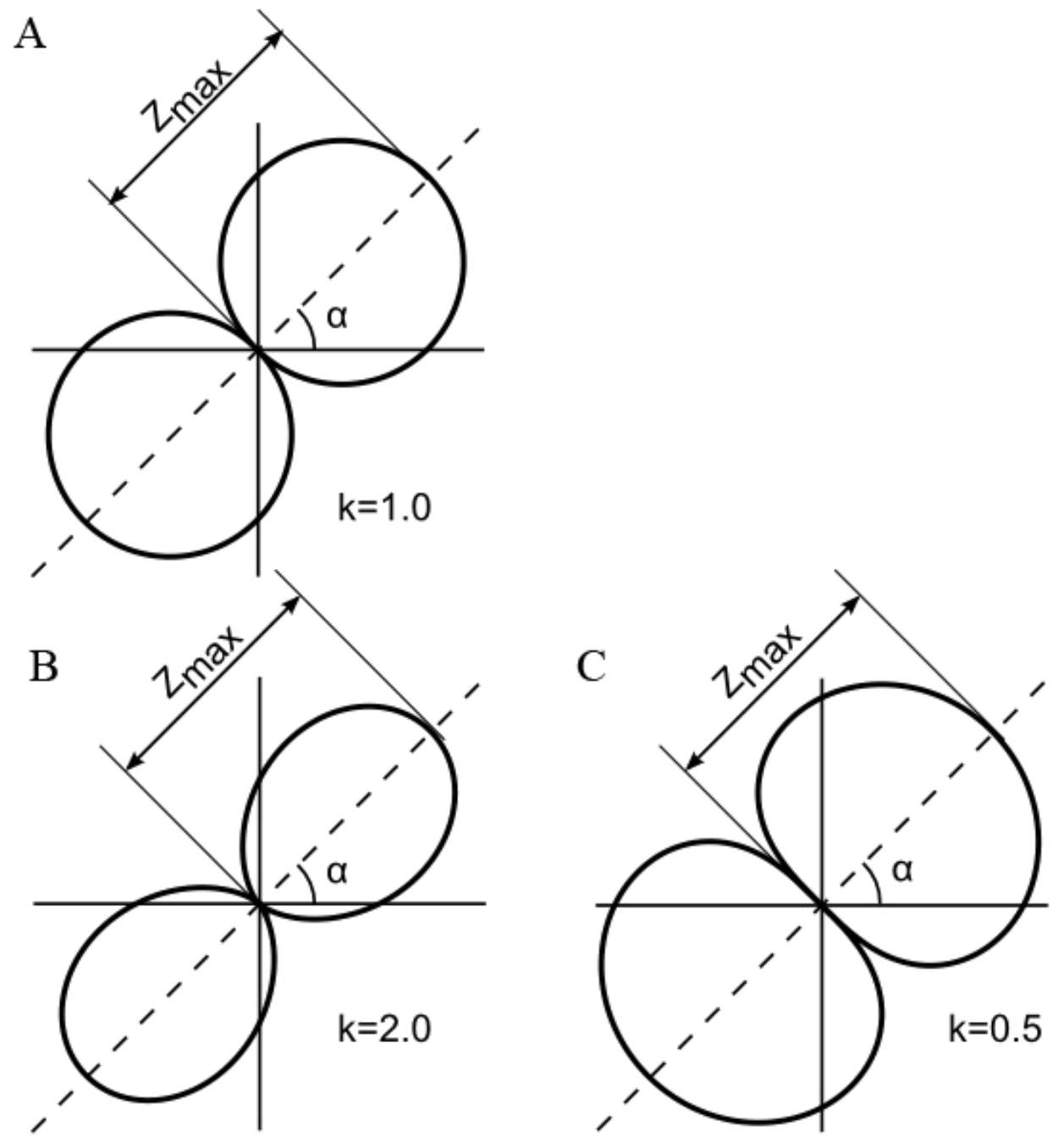

Fig. 4.1: Graphic representations of the model fitting different DRP-shapes ("cosine, "narrow", "flattened" DRP) when changing the parameter k (for formula and detailed explanation, see text). $\alpha=$ angle of best axis (BA), $\mathrm{Z}_{\max }=\operatorname{maximum} \mathrm{Z}$ value at $\mathrm{BA}$ for a given level. A: setting $\mathrm{K}$ to 1 results in a cosine-shaped function. B: setting $\mathrm{K}$ to 2 (or other values larger than 1 results in a "narrow-shaped" function. C: setting K to 0.5 (or other values smaller than 1) results in a "flattened" DRP shape. 

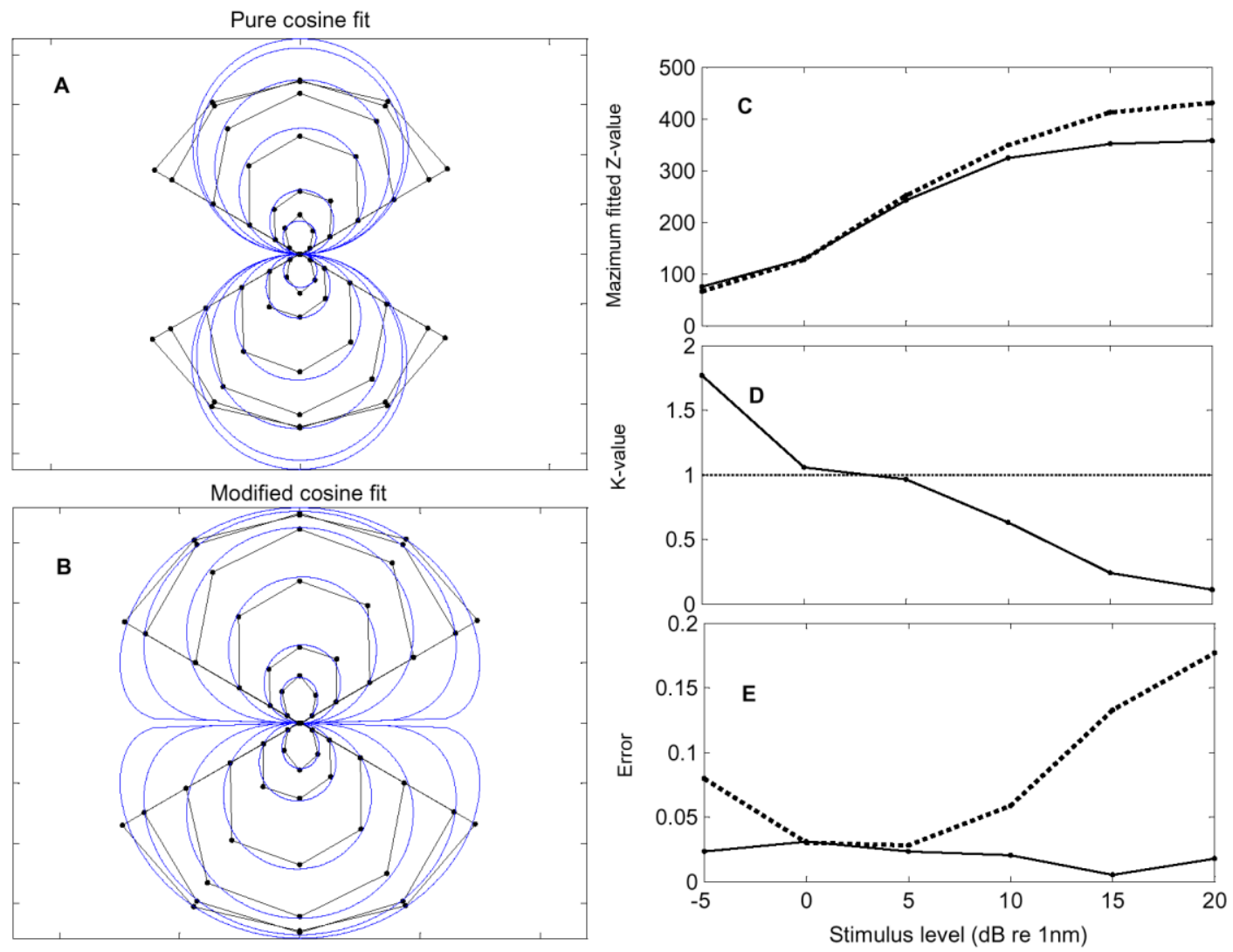

Fig 4.2: Example of a unit (pp18) fitted with a pure cosine fit (A), and the modified cosine fit $(\mathbf{B})$. C: $Z_{\max }$ as a function of stimulus level indicating the start of saturation of a unit. $Z_{\max }-$ values were obtained from the cosine fit (dotted line) and from the modified cosine fit (solid line). D: the $\mathrm{K}$-value is plotted as a function of stimulus level for the modified cosine fit. E: Errors are plotted for the cosine fit (dotted line) and the modified cosine fit (solid line) as a function of stimulus level. When $\mathrm{K}$ equals 1 the errors of the cosine and the modified cosine fit are equal. For this unit K equals 1 within the unit's dynamic range (at 0 and $5 \mathrm{~dB}$ re $1 \mathrm{~nm}$ ) and declines rapidly when the unit reaches saturation $(10,15 \mathrm{~dB})$. 

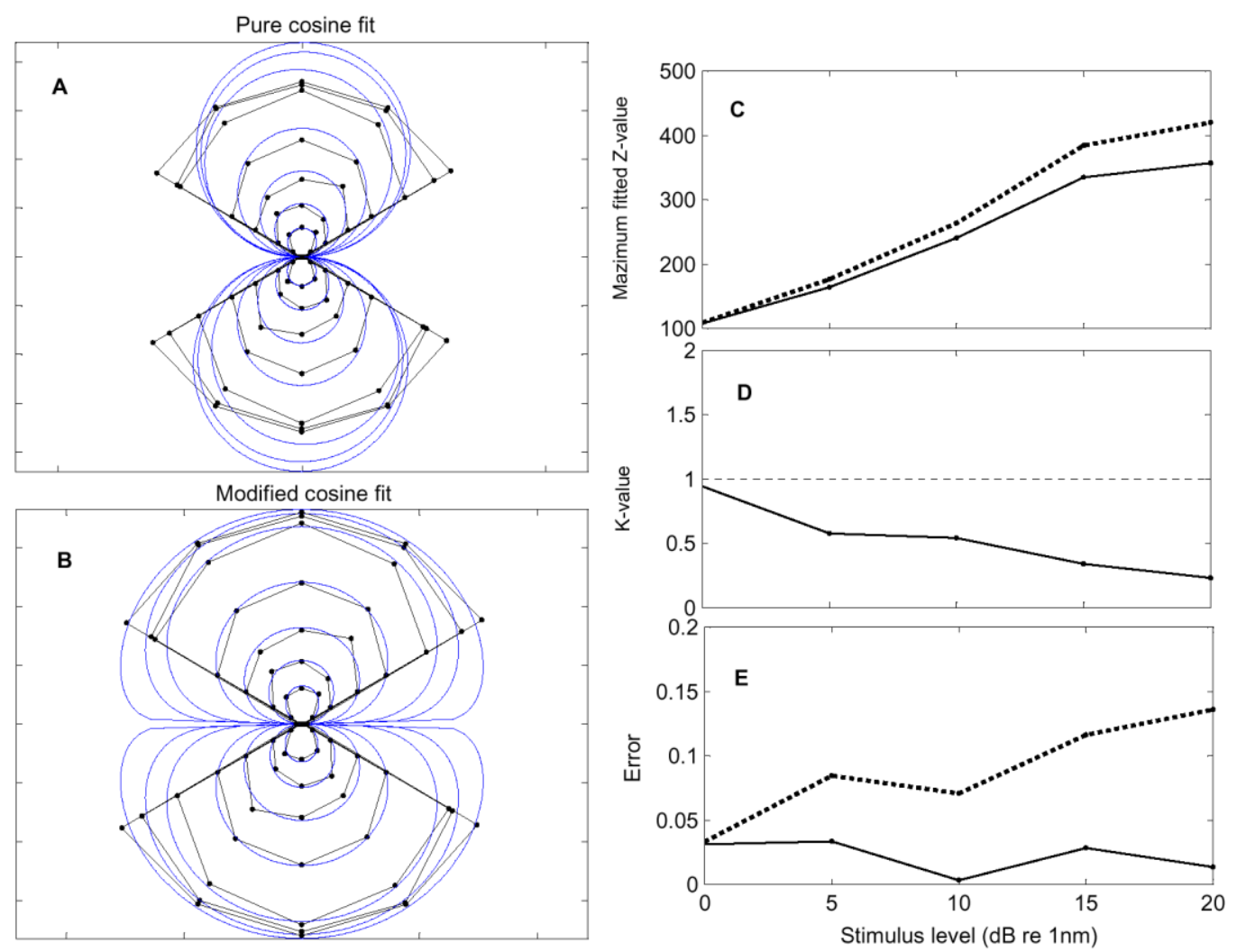

Fig 4.3: Example of a unit (tt7) fitted with a pure cosine fit (A), and the modified cosine fit $(\mathbf{B})$. C: $Z_{\max }$ as a function of stimulus level indicating the start of saturation of a unit. $Z_{\max }$-values were obtained from the cosine fit (dotted line) and from the modified cosine fit (solid line). D: the $\mathrm{K}$-value is plotted as a function of stimulus level for the modified cosine fit. E: Errors are plotted for the cosine fit (dotted line) and the modified cosine fit (solid line) as a function of stimulus level. For this unit most of the values for $\mathrm{K}$ are smaller than 1 indicating a more flattened shape even when unit is not in saturation yet ( 5 and $10 \mathrm{~dB}$ levels). 

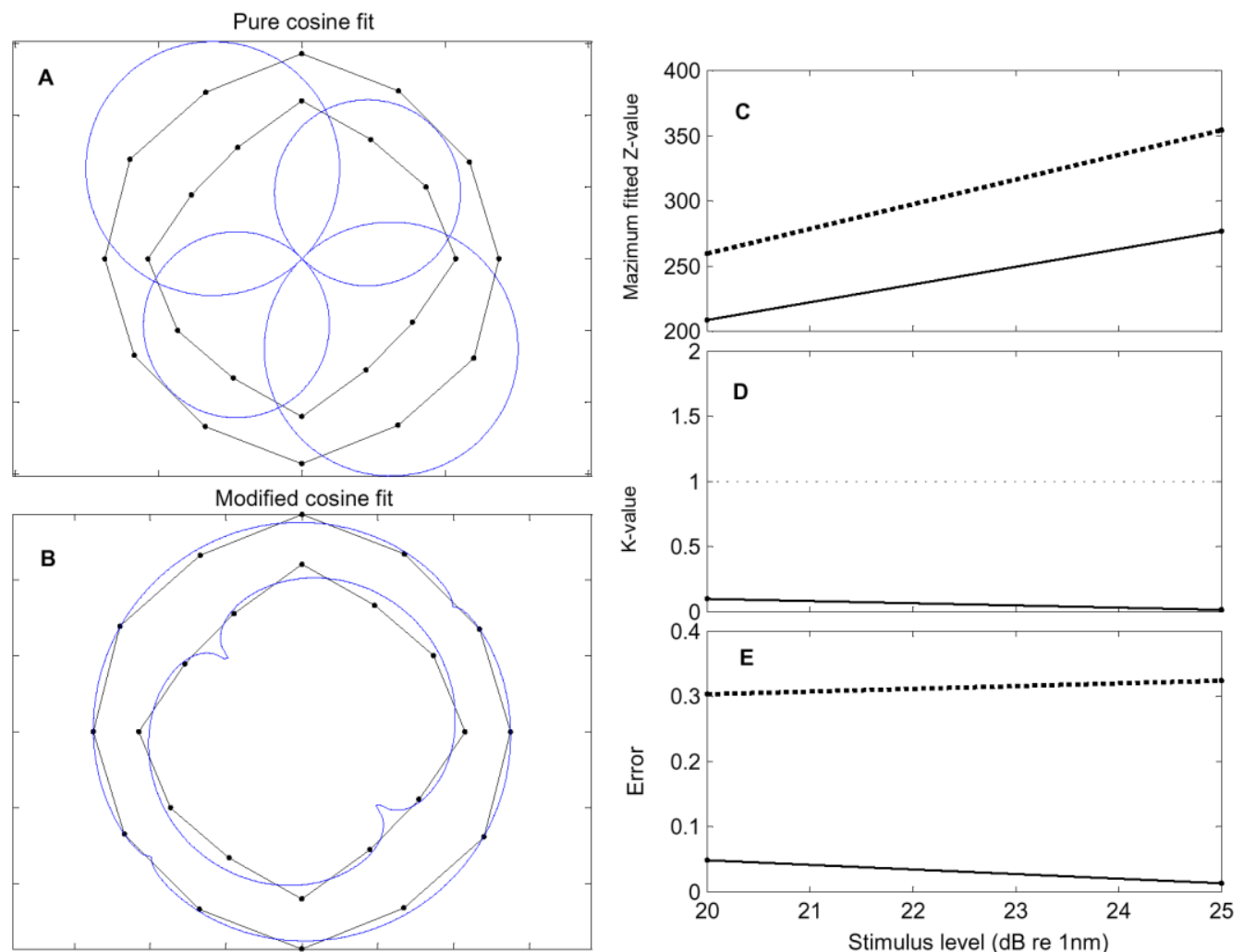

Fig 4.4: Example of an omnidirectional unit (ff1) fitted with a pure cosine fit (A), and the modified cosine fit (B). C: $Z_{\max }$ as a function of stimulus level indicating the start of saturation of a unit. $Z_{\max }$-values were obtained from the cosine fit (dotted line) and from the modified cosine fit (solid line). D: the K-value is plotted as a function of stimulus level for the modified cosine fit. K-values were very small for this unit. E: Errors are plotted for the cosine fit (dotted line) and the modified cosine fit (solid line) as a function of stimulus level. The modified cosine fit adjusted better to the non-directional response profile. 


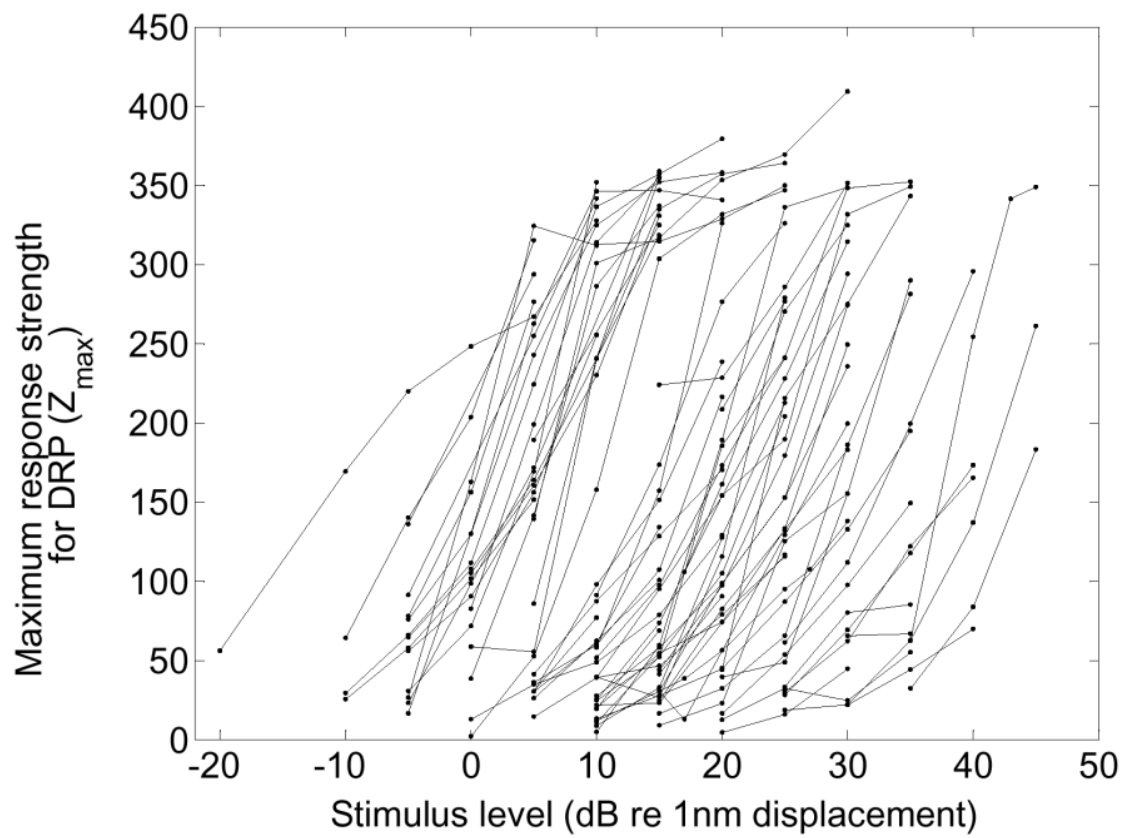

Fig. 4.5: Maximum Z-values (Z-values at BA) obtained from the modified cosine fit for each DRP is plotted as a function of stimulus level. Note that the dynamic range, slopes, and the spread in Z-values are similar but that afferents varied widely in threshold. Number of afferents: $\mathrm{N}=70$. 


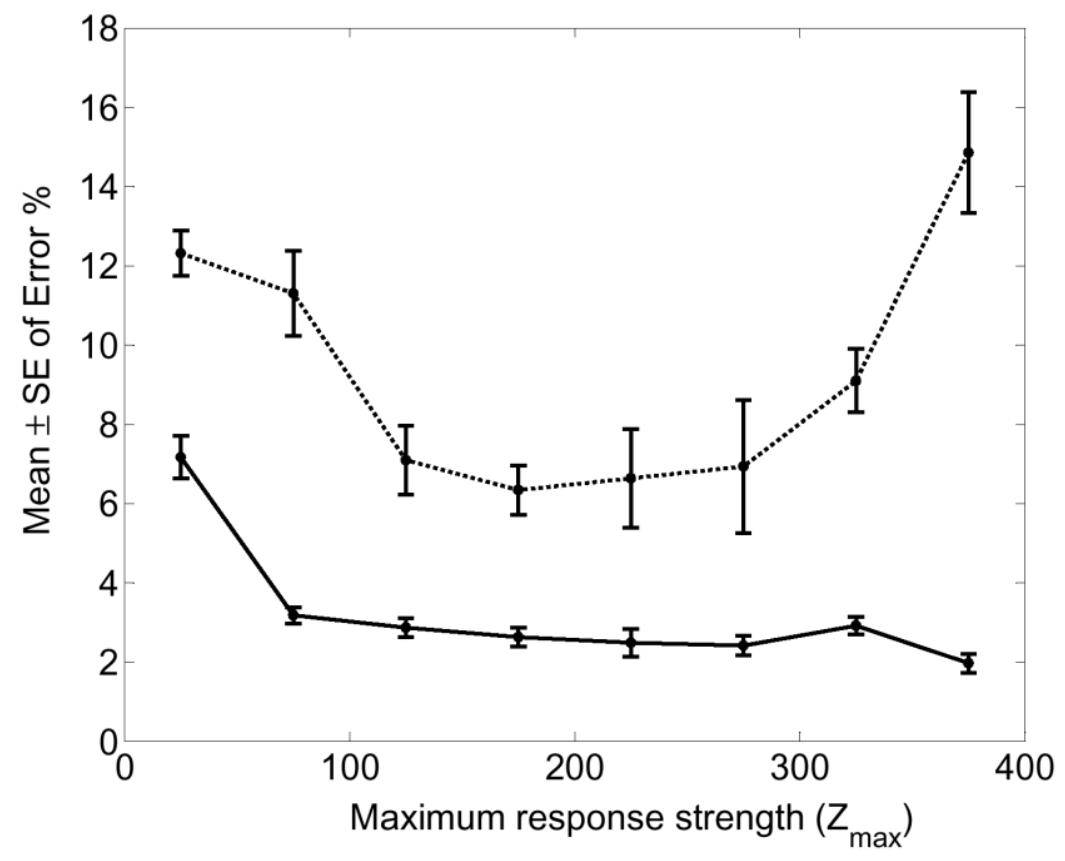

Fig. 4.6: The mean of the error is plotted for DRPs with maximum $Z$ values occurring at certain bins (bin size is 50). Error bars reflect the standard error (SE). The dotted line represents this relationship for the cosine fit and the solid line for the modified cosine fit. Since Z-values are usually proportional to stimulus level for each individual unit (e.g., higher Z-values are usually obtained at or near the unit's saturation point), the trends can also be interpreted as a function of level. Note the Ushape of the cosine fit, which indicates that the cosine fit results in high error-values at very low and very high Z-values (or levels). In contrast, the modified cosine fit works well at low and high Z-values (levels) and generally seems to be the better fit. The data are obtained for all DRPs (not for the non-directional response profiles), $\mathrm{N}=$ 282. 


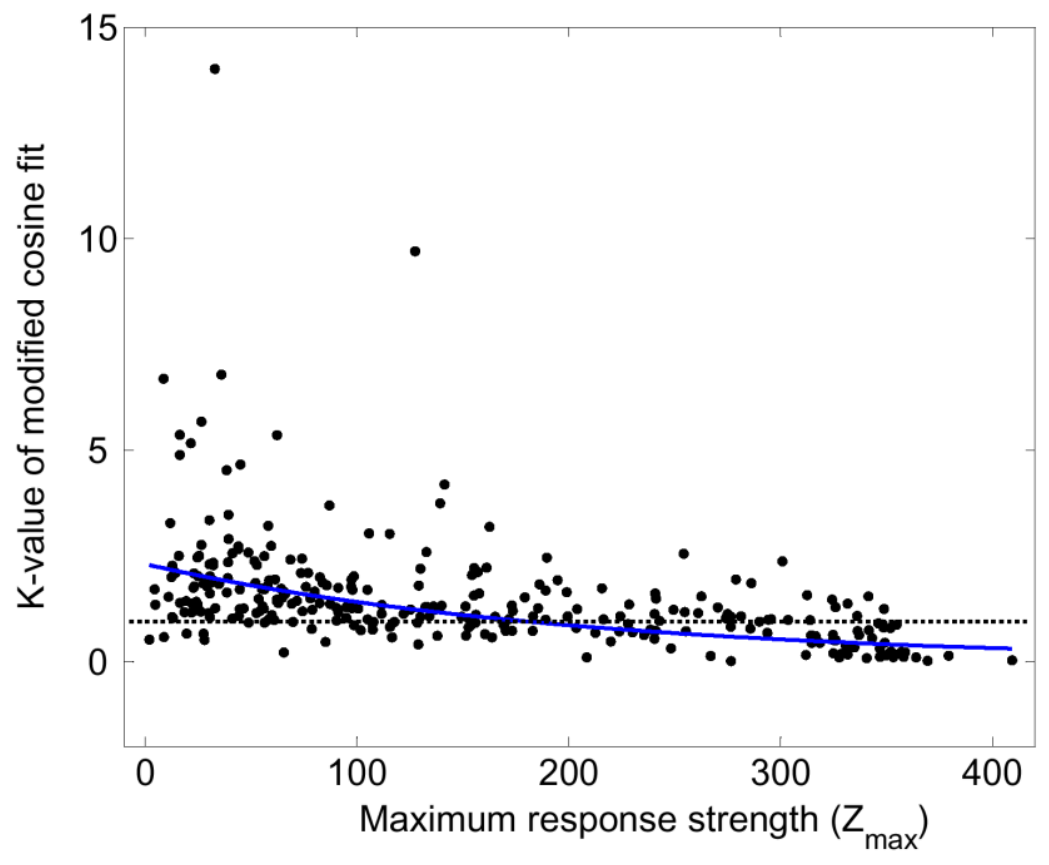

Fig. 4.7: The distortion factor or K-value of the modified cosine fit is plotted as a function of the maximum response strength for 74 afferents (282 DRPs). The solid blue line reflects the straight fit through the fitted DRP-data. With increasing Z-value, the K-value decreased exponentially (see blue line). Note that there are K-values smaller than 1 (flattened DRP-fits) also at low $\mathrm{Z}_{\max }$ values (levels) and $\mathrm{K}$-values larger than 1 (narrow DRP-fits) also at high $\mathrm{Z}_{\max }$ values (levels); the black dotted line marks 1 on the y-axis. 


\begin{tabular}{||l||l||l||}
\hline $\begin{array}{l}\text { Categories based on change of K with } \\
\text { level }\end{array}$ & $\begin{array}{l}\text { Number of } \\
\text { units }\end{array}$ & $\begin{array}{l}\text { Percentage } \\
\text { (out of 70 units) }\end{array}$ \\
\hline most Ks $>1$ within dynamic range of unit & 26 & $37 \%$ \\
K changes from <1 to $>1$ & 22 & $31 \%$ \\
most Ks $<1$ within dynamic range of unit & 21 & $30 \%$ \\
K = 1 for most levels & 1 & $2 \%$ \\
\hline
\end{tabular}

Table 4.1: Distribution of units according to certain categories that are based on how $\mathrm{K}$-values changed with level for DRPs.

Note: this work is done in collaboration with Kaushik Ghose.

The relative contributions have been: idea for modeling and testing effect of

level, context, and written text by MM - modified cosine fit, process of fitting

data, and idea for expressing effect as a function of $Z$ (graphs 6 and 7) by KG. 


\section{CHAPTER V}

\section{Summary, Final Discussion, and Conclusion}

\section{Summary of findings}

This dissertation investigated coding strategies by the auditory periphery in a non-teleost bony fish, Acipenser fulvescens (the lake sturgeon). Chapter 2 examined the coding strategies for frequency and intensity of sound. In addition, the frequency range and the intensity of sound that the saccule and lagena in a non-teleost bony fish detect were discussed. Chapter 3 focused on the directional coding strategies and the mechanisms for sound source location in A. fulvescens.

In Chapter 4, the directional response data obtained in Chapter 3 were described quantitatively with a mathematical model (a modified cosine fit). The fits of the directional response profiles (DRPs) to a pure cosine function (which responds to a response profile from a single hair cell, Hudspedth and Corey 1977) and a modified cosine function were compared. As a second goal, the effect of stimulus level on the directional response profile shape was investigated to test if shapedifferences occurred as a result of level or were caused by other processes (e.g., by slight differences in hair cell orientations that one afferent innervates).

In Chapter 2, a shaker table was used to simulate particle motion for stimulation of the ear and auditory periphery of $A$. fulvescens. Data were analyzed and discussed with respect to frequency selectivity, intensity responses, and temporal response pattern of eighth nerve fibers. Results showed that afferents strongly phase coupled to the stimulus waveform. Tuning curves were centered on a few different 
frequencies and there was no indication of a tonotopic organization during recordings. The shape of the tuning curves (or isolevel frequency response functions) and bandwidth of individual afferents varied. Eighth nerve responses occurred at a wide range of intensities, and all afferents had sigmoidal, monotonic response level functions with a similar spread in Z-values. There was variability in terms of the response pattern of afferents. The majority of fibers were tonic, but some fired in a phasic tonic fashion to the stimulus. Some of the tonic or phasic tonic fibers also showed bursting. Fibers with a phasic response pattern only occurred in a few afferents at high frequencies.

The hearing range to particle motion stimuli was estimated from best frequencies and maximal tuning curve widths in A. fulvescens and ranged between 50 and $700 \mathrm{~Hz}$. Lowest stimulus levels needed to evoke a response in eighth nerve fibers ranged between 100 and $300 \mathrm{~Hz}$. While the stimulus used in this dissertation was particle motion, previous recordings of auditory evoked potentials (AEP) used a loudspeaker submerged in water (and a pressure-based calibration) displaying pressure as well as particle motion of sound (Meyer et al. 2002). The results indicated responses up to about $2000 \mathrm{~Hz}$. The lowest levels needed to evoke a response in this AEP study occurred between 100 and $300 \mathrm{~Hz}$, thus confirming the results found in this dissertation using particle motion. At frequencies higher than $300 \mathrm{~Hz}$, stimulus levels needed to evoke an AEP-based response increased steeply. Responses to frequencies larger than $700 \mathrm{~Hz}$ in AEP-studies may be explained by pressure sensitivity of the swim bladder in A. fulvescens. While the swim bladder (which contains gas) is located with some distance to the ear in A. fulvescens (personal 
observation, Meyer and Ketten unpublished), it may still be possible that some of the sound pressure based energy that causes the swim bladder to pulsate in the sound field is directed to the ear in the form of (internal) particle motion (Sand and Hawkins 1973, Chapman and Sand 1974, Popper 1974). Studies of two scianid species with different swim bladder-inner ear configuration showed that the frequency range of detection was correlated with the proximity of the swim bladder to the ear (Ramcharitar et al. 2006). It was hypothesized that the gas filled swim bladder in teleosts can act like a secondary near-field source with particle motions that decays with the square of the distance to the bladder (in water) (Sand and Enger 1973). Interestingly, a cardiac conditioning study in Anguilla anguilla (European eel) showed that even in this species which has the swim bladder located in great distance to the inner ear (10 $\mathrm{cm}$ for a $50 \mathrm{~cm}$ long eel), the animals showed pressure sensitivity at higher frequencies (Jerko et al. 1989). The author concluded that the transmissionpath must have therefore been more efficient than water in A. anguilla.

Since higher frequencies propagate better in shallow waters (however within a shorter range - Rogers and Cox 1988), a detection of higher frequencies (as found in AEP studies for frequencies up to $2000 \mathrm{~Hz}$ ) may be useful for A. fulvescens during spawning in rivers (shallow waters).

Recordings were made from the posterior ramus of the right ear of $A$.

fulvescens. This ramus innervated the saccule, lagena, posterior crista, and the macula neglecta. It was impossible to access any distal portions of this ramus for recordings (for instance to access the small branch that just innervates the lagena) due to the tight connection of the nerve with the pouch containing the saccule and lagena without 
severely damaging those epithelia. In general, all results (with a few exceptions, see efferents, Chapter 3) were interpreted with regard to the assumption that afferents with frequency- and directional selectivity innervated most likely the saccule or lagena but not the posterior crista or macula neglecta. The latter two end organs do not contain otoliths or otoconia, but instead their hair cells are covered by a gelatinous mass (personal observation) and they are likely to respond to fluid motion at lower frequencies. Thus, these two end organs most likely did not respond to linear acceleration since they do not structurally resemble accelerometers (de Vries 1950, Retzius 1884). In addition, plotting tuning curves as a function of acceleration (see Chapter 2) illustrated that the tuning curve shapes appeared to be as expected from accelerometers (de Vries 1050, Kalmijn 1988, 1989).

Chapter 3 investigated the directional selectivity of primary eighth nerve afferents and provided distributions of thresholds and background rates. The information on spontaneous rate- and threshold-distribution was used to test the dependency of phase angle on level and background rate. Responses of eighth nerve afferents to directional stimuli provided by movement of the fish along defined axes in three dimensions were measured. Directionally selective afferents showed a directional response profile (DRP) that approximately resembled a cosine function with a best axis (BA) in the vertical and/or horizontal plane as seen in teleosts (Hawkins and Horner 1981, Fay and Edds-Walton 1997a, Lu et al. 1998). These cosine-like shapes appeared to be similar (but not equal) to the functions expected from single hair cell stimulation (Flock 1964, Hudspedth and Corey 1977) (Chapter 4 
presents a more detailed description of the cosine shapes). All of the directionally responsive afferents also showed frequency tuning such as described in chapter two. Some afferents had omni-directional response profiles (based on spike rate) and did not phase-lock to the stimulus. Some of those afferents looked like low-pass filters in their frequency tuning and therefore were classified as efferents (EddsWalton 1999, Weeg et al. 2002). The majority of afferents were directionally selective, especially to stimuli in the vertical plane. Generally, the best axes (BAs) obtained for the vertical plane clustered around $90^{\circ}$, although they varied in background activity and threshold. Afferents showing a BA at $90^{\circ}$ (up down axis of the fish; majority of afferents) would not respond to stimulation in the horizontal plane or they would respond to horizontal plane stimuli but only at high stimulus levels. Those afferents that showed a response in the horizontal plane had BAs mostly near $0^{\circ}$ (front back axis of the fish). Thus, BAs in the horizontal plane tended to lie parallel to the orientation of the saccule and lagena (similar to findings in teleosts, Fay 1984, Fay and Edds-Walton 1997a, Lu et al. 1998, 2003), which means along the midline of the fish in A. fulvescens.

Afferents varied widely in thresholds, with some fibers being extremely sensitive to the particle motion component of sound (showing phase-locked responses up to $0.07 \mathrm{~nm}$ ). Background rates and phase angles, at which afferents phase-locked, also varied among afferents. For most fibers, the phase angles of one afferent varied with stimulus intensity (only in a few fibers with very high spontaneous rates, the phase angle stayed constant with level). 
In Chapter 4, directional and non-directional response profiles of eighth nerve afferents were fitted with a model to categorize the shape of the directional response profile, capture the differences in DRP-shape, and investigate the effect of level on DRP-shape. It was found, that most shapes deviated slightly from a strict cosine shape and thus from the tuning of individual hair cells. Some DRPs looked narrower, others more flattened as compared to a cosine function. Thus, a modified cosine-fit using one parameter-value $\mathrm{K}$ that adjusted to these differences fitted the data better than a pure cosine-fit. While a K-value of one lead to a pure cosine function, Kvalues smaller than one caused the cosine-function to become more flattened. In contrast, K-values larger than one caused the cosine-function to be narrower. The correlation of $\mathrm{K}$ with the response strength $(\mathrm{Z})$ of a unit revealed that at lower $\mathrm{Z}$ values (corresponding to levels near threshold of the unit), the K-value was usually larger than one consistent with threshold effects for some stimulus angles. With higher Z-values (or with higher levels), $\mathrm{K}$ decreased, leading to more flattening of the DRP consistent with a tendency for saturation at the most effective axes. Thus, stimulus level had an effect on DRP-shape (as qualitatively described in the literature before, e.g., Weeg et al. 2002, Lu et al. 2003), however for many afferents, the Kvalue was smaller (indicating a more flattened shape) or larger even within the dynamic range of that unit indicative of level-independent effects at the level of the epithelia. 


\section{Research implications: functional and evolutionary insights}

\section{Functional implications}

Tuning curves (or isolevel frequency functions) varied in sensitivity, shape, and sharpness. Therefore, frequency coding may be possible through population coding performed by peripheral filters that vary slightly in $\mathrm{CF}$ (or BF) but show diversity in filter shape and sensitivity. A response pattern across a population of afferents representing stimulus features such as frequency and intensity may then be encoded centrally. Such a code based on a small number of differently tuned channels (also found in C. auratus and O. tau, Fay 1997, Fay and Edds-Walton 1997b) may not require tonotopicity (as seen in land vertebrates) and may be the most primitive (spike rate) code for spectral analysis in vertebrates (as suggested before for teleosts, e.g., Popper and Fay 1999). Frequency information in A. fulvescens could also be encoded by phase locking within the entire frequency range of hearing (temporal code, Fay 1974, Fay 1997, Fay and Edds-Walton 2008). Thus, spectral analysis in $A$. fulvescens is potentially based on a temporal as well as a rate code (it could be either one code that is used or both). With respect to intensity coding, the monotonic response level functions having different thresholds that covered a wide range of levels may be very well suited to encode sound intensities in A. fulvescens. Tonic fibers would be well suited in encoding ongoing sounds, while phasic tonic or phasic fibers (only found for frequencies higher than $300 \mathrm{~Hz}$ ) would be useful in encoding the onset of sounds. A. fulvescens had similar BFs to the teleosts species mentioned above which have been classified as hearing non-specialists except no afferents were 
found that indicated a strong response to frequencies smaller than $100 \mathrm{~Hz}$ in $A$. fulvescens.

In terms of the frequency range and sensitivity to particle motion stimuli of saccular and lagenar afferents, the results found in A. fulvescens, resemble those seen in teleost hearing non-specialists (Fay and Edds-Walton 1997, Weeg et al. 2002, Lu et al. 1998, 2003, 2004). A complete study on frequency responses of afferents innervating the saccule, lagena, and utricle respectively, are known for only one teleost species, Dormitator latifrons (sleeper goby, Lu et al. 1998, Buchser et al. 2003, Lu et al. 2003, Lu et al. 2004). While results in D. latifrons showed that saccular, lagenar, and utricular fibers have a similar range of characteristic frequencies, it would be useful to test the frequency selectivity of utricular afferents in A. fulvescens to see if there is such a similarity of frequency selectivity among eighth nerve afferents also.

Afferents innervating the saccule and lagena showed strong directional selectivity since all frequency-selective afferents responded in a cosine-like manner to variations in stimulus direction. Thus, the cosine-like tuning in A. fulvescens could represent the basic neural code of the peripheral auditory system used to compute the axis of particle motion emitted by a sound source.

In teleost fishes that have been used so far in shaker table experiments, the saccules and lagenae deviate from the midline of the fish at angles between $16^{\circ}$ and $40^{\circ}$ in azimuth (Fay 1984, Edds-Walton and Fay1997, Lu et al. 1998, Weeg et al. 2002, Lu et al. 2003). For any sound source, the stimulation of the right and left saccules (or lagenae) leads to differences in response magnitude arising from these 
epithelia in teleost fishes (Fay 1984, Fay 2005). It was proposed that such a difference in response magnitude could then be used to centrally compute the azimuth of a sound source (Sand 1974, Fay 1984, Fay and Edds-Walton 1997a). In contrast, best axes of afferents in the horizontal plane tended to be parallel to the orientation of the saccule and lagena in the horizontal plane in A. fulvescens. A parallel orientation, however, cannot give rise to interaural differences in response magnitude arising from the left and right saccules and lagenae. Maybe A. fulvescens uses auditory cues, such as arrival time differences to compute a sound source in azimuth or the axis of particle motion in azimuth is encoded by utricular fibers (since the utricle is oriented horizontally and carries hair cells oriented in various directions, Lovell et al. 2005).

The computation of the direction of a sound source in the vertical plane is possible yet somewhat limited due to the preference for $90^{\circ}$. Thus, other computational strategies may be used. One possibility would be a central comparison of inputs from afferents innervating the horizontally oriented utricle with inputs from the vertically oriented saccule and lagena to compute the location of a sound source in elevation. In addition, behavioral strategies may be used to compensate for the uniformity in BAs in elevation. To increase the chance of detecting a particular stimulus axis emitted from a sound source in the vertical, the animal could, for instance, change its body axis (as if redirecting the orientation of an acoustic fovea to get the best acoustic image "in focus"). In this way, encoding of sound in elevation would still be possible with one ear in A. fulvescens.

The variability in phase-angles found in A. fulvescens and teleosts (e.g., Fay and Edds-Walton 1997a) does not support the idea of solving the " $180^{\circ}$ - ambiguity - 
problem" as proposed by the phase model (using direct and indirect stimulation of the ear and computing the phase angle between a pressure-based input with the particle motion-based input of the signal; Schuijf 1975, Schuijf and Buwalda 1975). Behavioral adjustments (e.g., swimming in a direction that maintained a constant angle to the sound source, Kalmijn 1997) may be performed and input from other senses may be used to solve this problem, similar to humans that have difficulties in detecting a sound source located in front or at the back of the head.

Certain findings (such as the varying phase angles or the best axes for horizontal plane stimuli) used to discuss the mechanisms for sound source location in A. fulvescens add to the general discussion as to whether hypotheses that were widely discussed in the fish literature in the past (such as the phase-model to solve the $180^{\circ}$ ambiguity) are valid (variable phase angles were also found in $O$. tau, Fay and EddsWalton 1997a). In addition, the mechanism to compute the direction of a sound source in azimuth may be discussed more in the future (when conducting similar experiments in other fish species), placing more emphasis on the importance of the utricle and opening the discussion on a possible use of binaural cues if there is sufficient distance between the ears.

The mathematical characterization (and categorization) of response profiles in Chapter 4 provided two functional insights: one is, that stimulus level indeed lead to changes in DRP-shape, the other is that there is variation in DRP-shape among afferents, which is independent of level. The latter might reflect the fact that afferents innervate many hair cells that vary slightly in their orientation, leading to flattened DRP-shapes in afferents. 
Overall, the structural differences of the ear between A. fulvescens and teleosts (otoconia versus dense otoliths as the main difference), as well as the fact that animals used in this study are juveniles (between one and two years old), did not seem to create any difference in terms of basic coding mechanisms (for frequency or direction) or sensitivity of afferents seen in the auditory periphery of A. fulvescens when compared to teleosts.

\section{Evolutionary implications}

Recordings from eighth nerve afferents innervating the saccule and lagena in A. fulvescens showed differences but also many similarities to findings described for the auditory periphery in teleost fishes (see reviews on structure and function in teleosts, e.g.: Popper and Fay 1999, Fay and Edds-Walton 2008). Among those similarities were strong phase-coupling (temporal coding for frequency), single tone suppression, band-pass like tuning curves differing in shape, bandwidth, and CF (spike rate coding for frequency), variable response pattern, variable background activity -rates and -pattern, variable (including very low) thresholds in single afferents, and directional selectivity of afferents describing cosine-like response profiles (coding for directional stimuli). The similarities to teleosts are interesting considering that the Acipenseriformes broke off from the teleost radiation approximately 200 years ago.

In addition, the findings in A. fulvescens showed many similarities to auditory systems in land vertebrates. For example, the low thresholds found in A. fulvescens (lowest: $0.07 \mathrm{~nm}$ ) were comparable to thresholds of $0.2 \mathrm{~nm}$ to basilar displacement stimuli in the guinea pig and to thresholds as low as $0.1 \mathrm{~nm}$ during vibrational stimuli 
in Lithobates catesbeiana (American bullfrog; Koyama et al. 1982). Other similarities were the variation in background activity, variation in response pattern, and thresholds among auditory afferents (see reviews by Kiang 1965, Ruggero 1992, Manley 1992, Simmons et al. 2007). Sharpness and phase locking in A. fulvescens corresponded to low frequency afferents in mammals, reptiles, birds, and frogs (Kiang et al.1965, Manley 1990, Gleich and Manley 2000, Simmons et al. 2007). Both, a spike rate code, as well as a temporal code for frequency analysis were found in A. fulvescens and these two coding principles also coexist in auditory afferents in land vertebrates for frequencies lower than $4 \mathrm{kHz}$.

In terms of coding for sound source location, the peripheral system in fish is unique compared to other vertebrates since fish appear to extract the direction to a sound source at their periphery by sensing the particle motion component of sound. As a consequence, much of the processing for directional hearing in fish takes place peripherally. A. fulvescens does not represent an exception from this, since robust cosine-like tuning was found in afferents innervating the saccule and lagena.

Generally, the similarities of coding strategies (and other response properties) in A. fulvescens to teleosts and land vertebrates can be interpreted in two ways. One would be that the findings in A. fulvescens represent the ancestral condition for Osteichthyes (bony vertebrates including land vertebrates, Liem et al. 2001) due to the basal position (being closer to the common ancestor of vertebrates) of Acipenseriformes within the cladogram of vertebrates. To fully explore this view, it would be useful to investigate the coding strategies by the auditory periphery of other non-teleost bony fishes since $A$. fulvescens may have developed modern advanced 
characteristics during its own evolutionary course. In addition, it would be useful to conduct similar investigations in species belonging to the outgroup of bony fishes, such as cartilaginous fishes.

An alternative (or additional) view would be that the process of convergence resulted in similar coding strategies for sound source location and spectral analysis in distantly related species. This would indicate that there may be only a few coding strategies that a vertebrate auditory system is likely to evolve. However, it seems likely that similiarities occurred because the auditory system in vertebrates derived from a common ancestor. The assumption of common ancestry is based on the notion that certain auditory/vestibular structures are considered homologous, such as the vertebrate hair cell (Coffin et al. 2004) and on the observation that the gross morphology of the inner ears is very similar among vertebrates.

The vertebrate hair cell is at the basis of all vestibular and auditory sensory systems, as well as of the lateral line. Its origin can be dated back at least to the earliest vertebrates (agnatha, jawless fishes) if not to the earliest chordates (Coffin et al. 2004, Manley and Ladher 2008). The first inner ear with hair cells was found in Myxines (hagfishes), the sister group of vertebrates. The ears of hagfishes have one single semicircular canal with two crista ampulares and a sensory epithelium (Jarvik 1980). The lampreys (Petromyzon and Lampetra) are the living representatives of the earliest vertebrates and they possess an ear with two semicircular canals and a macula communis that is partially differentiated into several regions (Loewenstein et al. 1968). The functions of the ears in lampreys or hagfishes are not known, but it is assumed that they serve the detection of linear acceleration and gravity. 
Three semicircular canals, each oriented in a different plane, and three separated otolith organs were first found in the ears of elasmobranchs and the earliest bony fish. It was suggested that hearing arose from the same structures as vestibular systems (van Bergjick 1967, Ladich and Popper 2004). The first evidence for hearing and sound sensitivity to sound of the inner ear was found in elasmobranchs (Loewenstein and Roberts 1950, Myrberg et al. 1972, Nelson and Johnson 1976, Corwin 1981). Therefore it seems plausible that elasmobranchs most likely show similar coding strategies to ancestral bony fish and teleosts. The significance of hearing in Agnatha still remains to be investigated.

The selective pressures shaping auditory systems arose from the general need to accomplish certain basic functions, which are required for auditory scene analysis. It seems likely that having auditory systems fulfilling these basic tasks could provide animals with a selective advantage early in vertebrate evolution especially for animals that were moving freely in complex three-dimensional environments..

\section{Future studies}

This dissertation provided important functional and evolutionary insights into the mechanisms for spectral analysis and sound source location, major functions of vertebrate auditory systems, using Acipenser fulvescens. Unresolved questions remain, and new interesting questions emerge from this work. While some ideas for future studies have been mentioned before in connection with the discussion of the results, the following list provides an overview of those ideas including a few new ones. 
1. There was no dichotomy seen in the physiological data obtained in $A$. fulvescens, which could indicate a functional separation between the saccule and lagena. However, retrograde intracellular labeling studies combined with physiological recordings would indicate more clearly whether there is a functional separation between these two end organs. Inputs from the lagena versus the saccule could then be clearly separated and characterized. Using this technique, the innervation of any end organ can be investigated, which would include the determination of the number of hair cells innervated by one afferent (number of dendritic endings, dendritic arbor size), and the determination of diameters of fibers. If such studies are combined with labeling studies of hair cells (fluorescent labeling of ciliary bundles) the orientation, and other morphological characteristics of hair cells (e.g., ciliary bundle length) innervated by an individual afferent could be correlated with physiological characteristics.

2. The utricle could be involved in coding for sound source location in azimuth, thus it would be useful to undertake similar investigations used in this study to determine directional selectivity of utricular afferents. In addition, frequency selectivity of utricular afferents would fully answer the question to which extend all three otolith organs contribute to spectral analysis (or hearing) in $A$. fulvescens and in fish in general (since there are not many studies investigating the role of the utricle in fish).

3. To test whether inputs from different otolith organs are centrally computed, neuroanatomical investigations may be undertaken to answer this question. 
4. In A. fulvescens as in many teleost hearing non-specialists, the role of the swim bladder in audition is not known. Testing A. fulvescens for pressure sensitivity combined with deflation experiments can answer the question if the swim bladder is involved in hearing in this species.

5. Behavioral experiments under controlled stimulus conditions are necessary to test if $A$. fulvescens can indeed localize sound sources. There is a general lack of studies on sound localization experiments in fish using appropriately calibrated stimuli underwater (that reveal the field of particle motion).

6. Vocalization studies in members of the family Acipenseridae are ambiguous and sparse. Since there are insights into the mechanisms for hearing revealed in this dissertation, it may be a good start to undertake well-controlled vocalization studies in A. fulvescens instead of using species in which nothing is known about their hearing.

7. Generally, studies in adult $A$. fulvescens may be useful to fully explore hearing capabilities and mechanisms for hearing in this species (in case that there are differences between juvenile and adult animals).

8. Investigations of other non-teleost bony fish would be necessary to fully determine which mechanisms or characteristics of the auditory system might be evolutionary primitive and which are derived. Together with data obtained in this dissertation for A. fulvescens, results from other non-teleost bony fish could potentially describe the ancestral condition for Osteichthyes. 


\section{Conclusion}

This study has illustrated that there are similar coding strategies for spectral analysis and sound source location, as well as many other similar physiological characteristics typical for the auditory periphery between teleosts and a non-teleost bony fish, Acipenser fulvescens. In addition, many aspects found in the peripheral auditory system in A. fulvescens are similar to those found in land vertebrates.

It is remarkable that despite such a structural diversity at the level of the ears in vertebrates (even within taxa, e.g., among teleost fishes or reptiles), many response characteristics at the level of the auditory nervous system seemed to be shared among vertebrates. This might indicate that evolutionary pressures on auditory systems were similar throughout vertebrate evolution and that there may be only a few "neuronal building blocks" from which only a limited number of coding strategies can emerge. In contrast, there may generally be more ways to extract and transduce a signal from the environment leading to structural diversity with regard to vertebrate ears.

The similarities in coding strategies by the peripheral auditory system may also reflect a common origin of hearing in vertebrates. While the findings in $A$. fulvescens, may represent the ancestral condition for Osteichthyes, studies of species belonging to the sister group, the Chondrichthyans (and potentially also lampreys) would be useful to further investigate the origin of these coding strategies for vertebrates.

In summary, this dissertation provides important functional insights into the mechanisms for spectral analysis and sound source location, which are important tasks for auditory scene analysis, in a non-teleost bony fish (proximate view). By 
comparing all findings with teleost fish and land vertebrates, this dissertation also provided insights into the evolution of coding mechanisms in the auditory system of vertebrates in general and finally presents possible answers to a more ultimate question, such as why certain mechanisms have evolved.

\section{APPENDIX}

\section{Phase-locking of primary afferents}

Responses from most afferents showed strong phase locking (the tendency for spikes to occur at a particular phase of the sinusoidal stimulus) at all frequencies. For each unit from a subset of randomly selected afferents $(\mathrm{N}=80)$, we determined the maximum $\mathrm{R}$ (coefficient of synchronization) and Z-value (phase-locked spike rate) during $100 \mathrm{~Hz}$ stimulation (BF for most afferents) at a stimulus level between 10 and $15 \mathrm{~dB}$ (within the unit's dynamic range). Plotting the $\mathrm{Z}$-value $\left(\mathrm{Z}=\mathrm{NR}^{2}\right.$, where $\mathrm{N}$ is the number of spikes) as a function of $\mathrm{R}$ for each of the 80 units revealed that most units belonged to the category of strong phase-lockers having R-values exceeding 0.5 and Z-values larger than 20 based on their responses to $100 \mathrm{~Hz}$ stimuli at $10-15 \mathrm{~dB}$ above threshold (Fig. A1). When applying the Rayleigh statistic, a Z-value at 20 indicates a very low probability $(\mathrm{p}=0.0001)$ that spikes are randomly distributed.

All afferents phase-coupled to the stimulus waveform within the frequency range they detected and the R-value of most afferents (99\%; 79/80) belonged to the category of strong phase-lockers since their R-values exceeded 0.5. The same categorization of weak and strong phase-lockers was used for saccular and lagenar afferents in D. latifrons (Lu et al. 1998, Lu et al. 2003), and for midbrain cells of $O$. 
tau (Edds-Walton and Fay 2003). All phase-locking saccular afferents and 63\% of the phase locking lagenar afferents in D. latifrons were strongly phase-locked. Thus, data from A. fulvescens show more resemblance to the findings for the saccular afferents of $D$. latifrons. Only about one-third of the cells investigated in the midbrain of $O$. tau were significantly phase-locked.

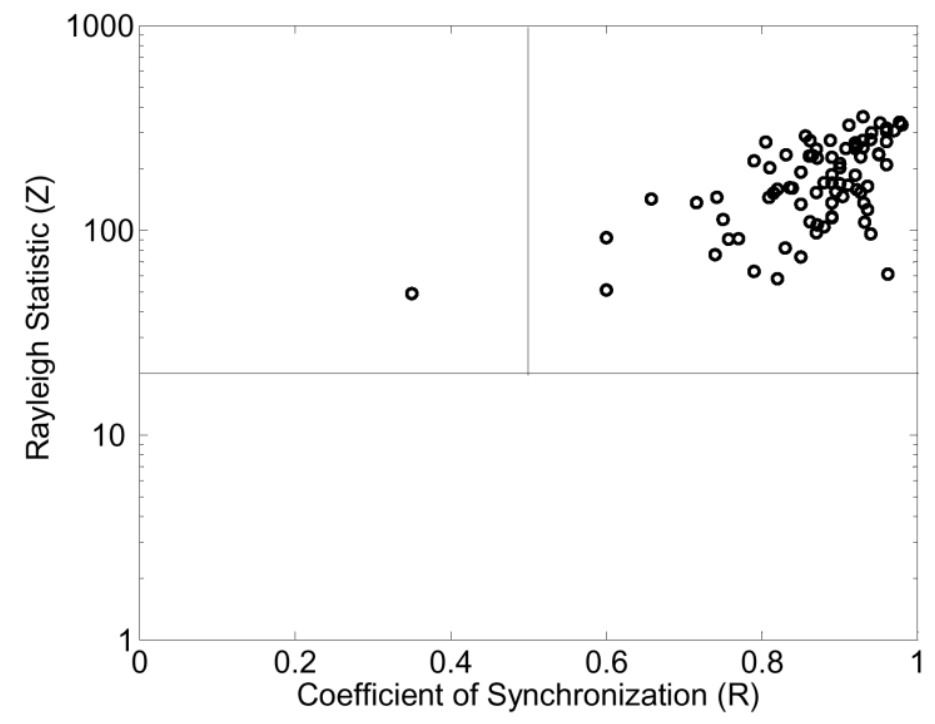

Fig. A1: Significance of phase locking among primary afferents $(\mathrm{N}=80)$ innervating the saccule or lagena in Acipenser fulvescens. The Rayleigh statistic $(\mathrm{Z})$ is plotted as a function of R, the coefficient of synchronization. Both values were determined at 100 $\mathrm{Hz}$ and 10 or $15 \mathrm{~dB}$ above threshold, within the unit's dynamic range. The horizontal line indicates the $\mathrm{Z}$-value $(\mathrm{Z}=20)$ above which phase locking is unlikely to be a random event $(P=0.0001)$. The vertical line $(R=0.5)$ separates weak (left) and strong phase-lockers (right). 


\section{Distributions of best frequencies across different}

\section{species}

The distributions of BFs among afferents between different species are compared in Figure A2. Afferents having the same BF (for the same species) were added up and expressed as a percentage of the total number of afferents tested. Frequencies at which most afferents had their BF would show the steepest part of the function (e.g., for A. fulvescens, most afferents had a BF at $100 \mathrm{~Hz}$, so the curve is steepest between 50 and $100 \mathrm{~Hz}$ ). The distribution functions for the utricle in $D$. latifrons and the saccule in P.notatus are similar except that a few afferents had BFs higher than $150 \mathrm{~Hz}$ in D. latifrons (Weeg et al. 2002, Lu et al. 2004). The data for the utricle in $D$. latifrons apparently are similar to data from the saccule and lagena in $D$. latifrons according to Lu et al. (2004), however distribution data were only available for the utricle. In A. fulvescens, most afferents responded best at $100 \mathrm{~Hz}$ and no afferents responded best at $50 \mathrm{~Hz}$ or lower (unlike seen in D. latifrons and $P$. notatus). There were only a few afferents in the lagena of C.auratus that responded to frequencies between 50 and $100 \mathrm{~Hz}$ (but more than in A. fulvescens). Most afferents had their BF near $200 \mathrm{~Hz}$ in the lagena of C. auratus (Meyer et al. 2004). Thus, the BF-distribution of $A$. fulvescens is in between the data for the saccule and utricle of $P$. notatus (green function) and D. latifrons (blue function) and the lagena of C. auratus (red function), however with a tendency to be more similar to $P$. notatus and $D$. latifrons (shifted more towards the blue and green functions). 


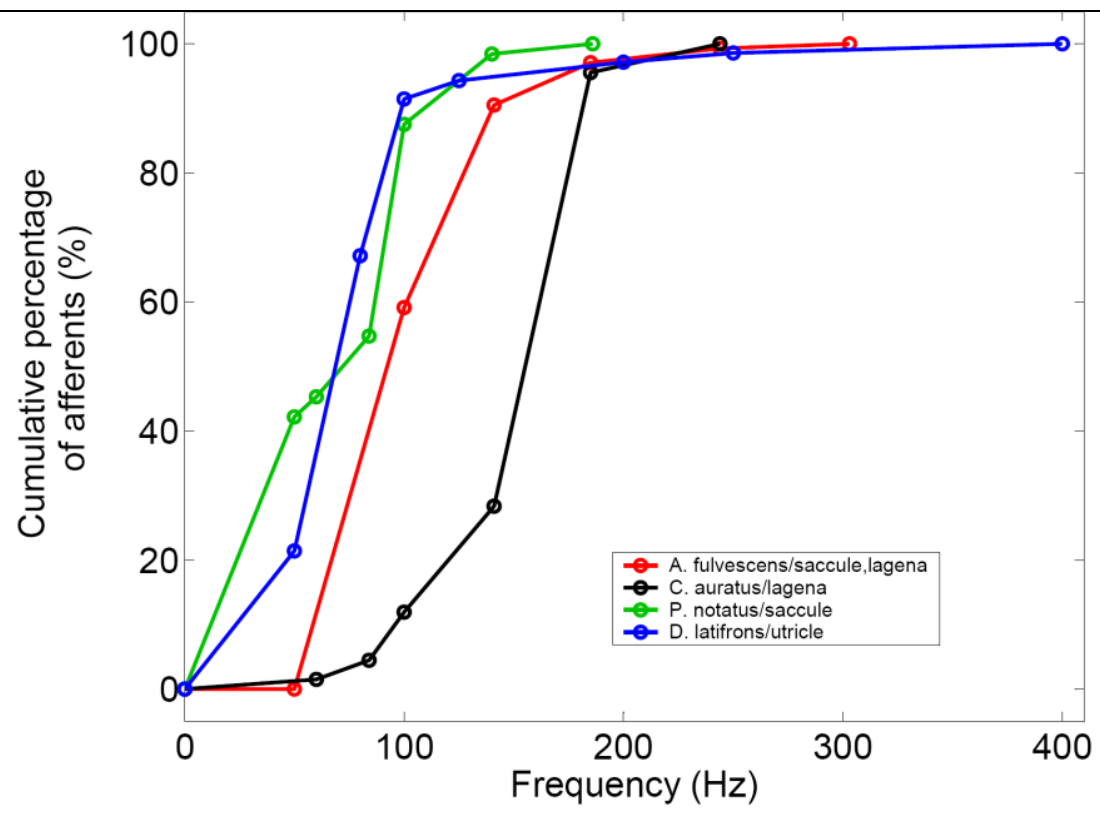

Fig. A2: Cumulative percentage of afferents having their $\mathrm{BF}$ at certain frequencies for the saccule and lagena in A. fulvescens $(\mathrm{N}=137)$, the lagena in C. auratus $(\mathrm{N}=67)$, the saccule in $P$. notatus $(\mathrm{N}=64)$, and the utricle in D. latifrons $(\mathrm{N}=70)$. Data for $D$. latifrons modified from Lu et al. 2004, for P. notatus from Weeg et al. 2002, data for C. auratus from Meyer et al. 2004. 


\section{Non-responsive afferents}

Many afferents showed no interpretable response to any change in the stimulus (direction, frequency, intensity). Those units always had a background rate that was very regular in its firing pattern. One example is shown in Figure A3. The signal noise ratio during recordings was excellent and this was a single unit recording.

Fig. A3: Example of a unit that did not show any interpretable response to stimulation




\section{LITERATURE CITED}

Allen, J. 1996. OHCs shift the excitation pattern via BM tension. In: Lewis, E.R. (Ed.) Diversity in auditory mechanics. World Scientific Publishers, Singapore, in press.

Batschelet, E. 1981. Circular statistics in biology. Academic Press, New York.

Bemis, W.E., Findeis, E.K., Grande, L. 1997. Diversity and evolution of sturgeon and paddlefishes. An overview of Acipenseriformes. Env. Biol. of fishes 48: 25-71, 1997.

Bemis, W.E., Kynard, B. 1997. Sturgeon rivers: an introduction to acipenseriform biogeography and life history. Environmental Biology of Fishes 48: 167-183.

Birstein, V.J. 1993. Sturgeons and paddlefishes: threatened fishes in need of conservation. Cons. Biol. 7:773-787.

Bolker, J. 2004. Embryology. In: Le Breton, G.T.O., Beamish, F.W.H., McKinley, R.S. (Eds.) Sturgeon and Paddlefish of North America. Springer-Verlag, New York, pp. 134-144.

Bregman, A. 1990. Auditory scene analysis: The perceptual organization of sound. MIT Press, Cambridge.

Bregman, A. 2008. Auditory scene analysis. In: Basbaum A.I., Kaneko A., Shepherd G. M., Westheimer G. (Eds.), The Senses: A Comprehensive Reference. Vol. 3, Auditon, Dallos, P., Oertel D. San Diego: Academic Press. pp. 861-870.

Buchser, W.J., Lu, Z., Xu, Z., Evoy, W.H. 2003. Frequency response of saccular afferents in a teleost fish. Soc. Neurosci. Abstr 93.7

Carlström, D., 1963. A crystallographic study of vertebrate otoliths. Biol. Bull.,125: 441-463.

Christensen-Daalsgard, J., Jorgensen, M.B., Kanneworff, M. 1998. Basic response characteristics of auditory nerve fibers in the grassfrog (Rana temporaria). Hear.Res.199:155-163.

Coffin, A. B., Kelley, M., Manley, G., Popper, A.N. 2004. Evolution of sensory hari cells. In: Evolution of the vertebrate auditory system. Springer-Verlag, New York, pp. 27-54. 
Coffin, A. B. 2005. Unconventional Myosins in fish ears. PhD dissertation, University of Maryland, p. 4.

Coombs, S.L., and Popper, A.N. 1982. Comparative frequency selectivity in fishes: Simultaneously and forward-masked psychophysical tuning curves. J. Acoust. Soc. Am. 71: 133-141.

Corwin, H.T. 1977. Morphology of the macula neglecta in sharks of the genus Carcharhinus. J. Morphol. 152: 341-362.

Corwin, H.T. Audition in elasmobranchs. In: Tavloga W.N., Popper, A.N., Fay, R.R. (eds.) Hearing and Sound Communication in Fishes. New York: Springer Verlag, pp. 81-102.

Dale, T 1976. The labyrinthine mechanoreceptor organs of the cod (Gadus morhua, Teleostei: Gadidae). Norw.J.Zool. 24:85-125.

Dallos, P., Harris, d. 1978. Properties of auditory nerve responses in absence of outer hair cells. J. Neurophysiol. 41:365-382.

de Vries, H.L.1950. The mechanics of the labyrinth otoliths. Acta Oto-Laryngol. 38: 262-273.

Eatock, R.A., Manley, G., Pawson, L. 1981. Auditory nerve fiber activity in the tokay gecko: implications for cochlear processing. J. Comp. Physiol. A. 142:203-218.

Eatock, R.A., Weiss, T.F. 1986. Relation of discharge rate to sound pressure level for chochlear nerve fibers in the alligator lizard. Abstracts $9^{\text {th }} \mathrm{Mtg}$. Assoc.Res.Otolaryngol., pp.63-64.

Edds-Walton, P.L., Highstein, S.M., Fay, R.R. 1996. Characteristics of directional auditory afferents in the toadfish, Opsanus tau. Soc. Neurosci. Abstr. 178: 447.

Edds-Walton, P.L., Fay, R.R., Highstein, S.M. 1999. Dendritic arbors and central projections of physiologically characterized auditory fibers from the saccule of the toadfish (Opsanus tau). J. Comp. Neurol. 411: 212-238.

Edds-Walton, P.L., Fay, R.R., 2003. Directional selectivity and frequency tuning of midbrain cells in the oyster toadfish, Opsanus tau. J. Comp. Physiol. 189:527543.

Enger, P.S. 1963. Single unit activity in the peripheral auditory system of a teleost fish. Acta. Physiol. Scand. 59: (suppl. 210): 9-48. 
Enger, P.S. 1966. Acoustic threshold in goldfish and its relation to the sound source distance. Comp. Biochem. Physiol. 18:859-868.

Fay, R.R. 1969. Behavioral audiogram for the goldfish. J. Aud. Res. 9: 112-121.

Fay, R.R. 1978a. Coding of information in single auditory nerve fibers of the goldfish. J. Acoust. Soc. Am. 63:136-146.

Fay, R.R. 1978b. Phase-locking in goldfish saccular nerve fibers accounts for frequency discrimination capacities. Nature. 275:320-322.

Fay, R. R. 1981. Coding of acoustic information in the eighth nerve. In: Hearing and Sound Communication in fishes. In: Tavolga, W.N., Popper, A.N., Fay, R.R. (Eds.), Hearing and Sound Communication in fishes. Springer-Verlag, New York, pp. 189-219.

Fay, R.R. 1984. The goldfish ear codes the axis of particle motion in three dimensions. Science 225: 951-953.

Fay, R.R. 1988. Hearing in vertebrates: A psychophysics databook. Hill-Fay Associates, Winnetka IL.

Fay, R.R. 1990. Suppression and excitation in auditory nerve fibers of the goldfish, Carassius auratus. Hear. Res. 48: 93-110.

Fay, R.R. 1997. Frequency selectivity of saccular afferents of the goldfish revealed by revcor analysis. In: Lewis, E.R., Long, G.R., Lyon, R.F., Narins, P.M., Steele, C.R., Hecht-Poinar, E. (Eds.), Diversity in Auditory Mechanics, World Scientific Publishers, Singapore, pp. 69-75.

Fay, R.R. 1998. Auditory stream segregation in goldfish (Carassius auratus). Hear. Res. 120, 69-76.

Fay, R.R. 2000. Spectral contrasts underlying auditory stream segregation in goldfish (Carassius auratus). J. Assn. Res. Otolaryngol. 1:120-128.

Fay, R.R. 2005. Sound localization by fishes. In: Popper, A.N., Fay, R.R. (eds.). Sound Source Localization. Springer Verlag, New York, pp. 36-66.

Fay, R.R., Popper, A.N. 1974. Acoustic stimulation of the ear in goldfish, Carassius auratus. J.Exp.Biol. 61:243-260.

Fay, R.R., Popper, A.N. 1975. Modes of stimulation of the teleost ear. 62: 379-387. 
Fay, R.R., Coombs, S. 1983. Neural mechanisms in sound detection and temporal summation. Hear. Res. 10: 69-92.

Fay, R.R., Ream, T.J. 1986. Acoustic response and tuning in saccular nerve fibers of the goldfish (Carassius auratus). J. Acoust. Soc. Am. 79:1883-1895.

Fay, R.R., Feng, A. 1987. Directional hearing among non-mammalian vertebrates. In: Yost, W., Gourevitch, G. (Eds.), Directional Hearing. Springer-Verlag, New York, pp. 179-213.

Fay, R.R., Edds-Walton, P.L. 1997a. Directional response properties of saccular afferents of the toadfish, Opsanus tau. Hear. Res. 111, 1-21.

Fay, R.R., Edds-Walton, P.L. 1997b. Diversity in frequency response properties of saccular afferents of the toadfish (Opsanus tau). Hear. Res. 113, 235-246.

Fay, R.R., Edds-Walton, P.L. 1999. Sharpening of directional auditory input in the descending octaval nucleus of the toadfish, Opsanus tau. Biol.Bull. 197:240241.

Fay, R. R., and Popper, A. N. 2000. Evolution of hearing in vertebrates: The inner ears and processing. Hear. Res., 149:1-10.

Fay, R.R., Edds-Walton, P.L. 2001. Bimodal units in the torus semicircularis of the toadfish (Opsanus tau). Biol.Bull. 201:280-281.

Fay, R.R., Edds-Walton, P.L. 2003. Sharpening of directional responses along the auditory pathway of the oyster toadfish, Opsanus tau. J.Comp.Physiol.A 191:1079-1086.

Fay, R.R., Edds-Walton, P.L. 2008. Structures and functions of the auditory nervous system of fishes. In: Webb, J.F., Fay, R.R., Popper, A.N. (Eds.), Fish Bioacoustics, Springer-Verlag, New York, pp. 49-98.

Findeis, E.K. 1991. Ontogeny and homology of the 'maxilla' of sturgeons: evolution of mobile jaw elements in early actinopterygian fishes. Amer.Zool.31:8A (abstract)

Findeis, E.K. 1993. Skeletal anatomy of the North American Shovelnose sturgeon Scaphorynchus platorhynchus (Rafinesque 1820) with comparisons to other Acipenseriformes. Ph.D. Dissertation, University of Massachusetts, Amherst. $444 \mathrm{pp}$.

Findeis, E.K. 1997. Osteology and phylogenetic interrelationships of sturgeons (Acipenseridae). Env.Biol.Fish. 48: 73-126. 
Fish, J.F., Offutt, G. 1972. Hearing thresholds from toadfish, Opsanus tau, measured in the laboratory and field. J. Acoust. Soc. Am. 51, 1314-1321.

Flock, A. 1964. Structure of the macula utriculi with special reference to directional interplay of sensory responses as revealed by morphological polarization. J. Cell Biol. 22:413-431.

Furukawa, T., Ishii, Y. 1967. Neurophysiological studies on hearing in goldfish. J. Neurophysiol., 30:1377-1403.

Gnatzie, W., Tautz. J. (1980). Ultrastructure and mechanical properties of an insect mechanoreceptor: stimulus-transmitting structures and sensory apparatus of the cercal filiform hairs of Gryllus. Cell Tissue Res. 213:441-463.

Gauldie, R.W. 1996 Fusion of otoconia: a stage in the development of the otolith in the evolution of fishes. Acta. Zool. 77: 1-23.

Gardiner, B.G. 1984. Sturgeon as living fossils. In: Eldredge, N., Stanley, S.M. (Eds.). Living Fossils. Springer Verl. New York,pp. 148-152.

Gardiner, B.G. 1993. Basal actinopterygians. In: Benton, M.J. (Ed.). The Fossils. Vol.2. Chapman \& Hall, London.

Gleich, O., Manley, G. 2000. The hearing organ of birds and Crocodilia. Dooling, R.J., Popper, A.N., Fay, R.R. (eds.) Comparative hearing: birds and reptiles. Springer-Verlag, New York, pp. 70-138.

Goldberg, J.M., Brown, P.B. 1969. Response of binaural neurons of dog superior olivary complex to dichotic tonal stimuli: some physiological mechanisms of sound localization. J. Neurophysiol. 32: 613-636.

Grande, L., Bemis, W.E. 1991. Osteology and phylogenetic relationships of fossil and recent paddlefishes (Polyodontidae) with comments on the interrelationships of Acipenseriformes. J.Vert.Paleo. 11, supplement 1:1-121.

Grande, L., Bemis, W.E. 1996. Interrelationships of Acipenseriformes, with comments on Chondrostei. In: M.L.J. Stianssny, L.R. Parenti and G.D. Johnson (Eds.) Interrelationships of Fishes, Academic Press, San Diego, pp. 85-115.

Hawkins, A.D., Horner, K. 1981. Directional characterstics of primary auditory neurons from the cod ear. In: Tavolga, W.N., Popper, A.N., Fay, R.R. (eds.) Hearing and Sound Communication in fishes. Springer-Verlag, New York, pp. 311-328. 
Horner, K., Hawkins, A.D., Fraser, P.J. 1981. Frequency Characteristics of Primary Auditory Neurons from the Ear of the Cod. Gadus morhua L. In: Tavolga, W.N., Popper, A.N., Fay, R.R. (Eds.), Hearing and Sound Communication in fishes. Springer-Verlag, New York, pp. 223-241.

Hudspeth, A.J., Corey, D.P., 1977. Sensitivity, polarity and conductance change in the response of vertebrate hair cells to controlled mechanical stimuli. Proc. Natl. Acad. Sci. USA 74:2407-2411.

Hulse, S.H., MacDougall-Shackelton, S.A., Wisniewski B. 1997. Auditory scene analysis by songbirds: Stream segregation of birdsong by European starlings (Sturnus vulgaris). J. Comp. Psychol., 111, 3-13.

Irvine, D.R.F. Physiology of the auditory brainstem. 1992. In: Popper, A.N., Fay, R.R. (Eds.). The mammalian auditory pathway: Neurophysiology. SpringerVerlag, New York, pp. 153-231.

Jacobs, D.W., Tavolga, W.N. 1968. Acoustic frequency discrimination in the goldfish. Anim. Behav. 16, 67-71.

Jarvik, E. 1980. Basic structure and evolution of vertebrates. Vol.1. London: Academic Press.

Jerko, H., Turunen-Rise, I., Enger, P.S., Sand, O. 1989. Hearing in the eel (Anguilla). J.Comp.Physiol. 165A:455-459.

Johnston, C.E., Phillips, C.T., 2003. Sound production in sturgeon: Scaphirhynchus albus and S. platorynchus (Acipenseridae). Environ.Biol.Fishes 68:59-64.

Kalmijn, A.J. 1988. Hydrodynamic and acoustic field detection. In: Atema J., Fay R.R., Popper A.N., Tavolga W.N. (Eds.) Sensory biology of aquatic animals. New York: Springer-Verlag, pp. 83-130.

Kalmijn, A.J. 1989. Functional evolution of lateral line and inner ear sensory system. In: Coombs, S., Görner, P., Münz, M. (Eds.) The mechanosensory lateral line: neurobiology and evolution. Berlin: Springer-Verlag, pp. 187-215.

Kalmijn, A.J. 1997. Electric and near-field acoustic detection, a comparative study. Acta. Physiol. Scand. 161 (Suppl 638): 25-38.

Kiang, N.Y.S., Watanabe, T., Thomas, C., Clark, L.F. 1965. Discharge patterns of single fibers in the cat's auditory nerve. Cambridge, MA: MIT Press.

Kleerekoper, H., Chagnon, E.C. 1954. Hearing in fish with special reference to Semotilus atromaculatus. J. Fish. Res. Bd. Can. 11: 130-152. 
Konishi, M., 1973. How the owl tracks its prey. Am. Scientist 61: 141-424.

Koyama, H., Lewis, E.R., Leverenz, E.L., Baird, R.A. 1982. Acute seismic sensitivity of the bullfrog ear. Brain Res. 250, 168-172.

Krieger, J., Fuerst, P.A., Cavender, T.M. 1999. Phylogenetic relationships of the North American Sturgeon (Order Acipenseriformes) based on mitochondrial DNA sequences. Mol. Phyl. and Evol., 16: 64-72.

Lewis, E.R., Narins P.M 1999. The acoustic periphery of amphibians: anatomy and physiology. In: Popper, A.N., Fay, R.R. (Eds.), Comparative Hearing: fish and amphibians. Springer-Verlag, New York, pp. 101-154.

Lewis, E.R., Fay R.R. 2004. Environmental variables and the fundamental nature of hearing. In: Manley, G. A., Popper, A.N., Fay, R.R. (Eds.), Evolution of the vertebrate auditory system. Springer-Verlag, New York, pp. 27-54.

Liberman, M.C. 1978. Auditory nerve responses from cats raised in a low-noise chamber. J. Acoust. Soc. Am. 63:442-455.

Liem, K.F., Bemis, W.E., Walker, W.F. Jr., Grande, L. 2001. Functional Anatomy of the Vertebrates. An evolutionary perspective. $3^{\text {rd }}$ Edition. Harcourt college Publishers, Orlando.

Löwenstein, O., Roberts, T.D.M. 1950. The equilibrium function of the otolith organs of the thornback ray (Raja clavatra). J.Physol. (Lond.) 110: 392-415.

Löwenstein, O., Osborne, M.P., Wersäll, J. 1964. Structure and innervation of the sensory epithelia of the labyrinth in the thornback ray (Raja clavata). Proc. Roy. Soc. Lond., B 160: 1-12.

Löwenstein, O., Osborne, M.P., Thornhill, R.A. 1968. The anatomy and ultrastructure of the labyrinth of the lamprey (Lampetra fluviatilis L.). Proc $\mathrm{R}$ Soc Lond B 170: 113-134.

Lovell, J.M., Findlay, M.M., Moate, R.M., Nedwell, J.R., Pegg, M.A. 2005. The inner ear morphology and hearing abilities of the paddlefish (Polyodon spathula) and the lake sturgeon (Acipenser fulvescens). Comp. Biochem. Physiol.A. 142: 286-296.

Lu, Z., Fay, R.R. 1993. Acoustic response properties of single units in the torus semicircularis of the goldfish, Carassius auratus. J. Comp. Physiol, 173: 33-48.

Lu, Z., and Fay, R.R. 1996. Two-tone interaction in auditory nerve fibers and midbrain neurons of the goldfish, Carassius auratus. Auditory Neuroscience, 2: $257-273$. 
Lu, Z., Popper, A.N. 1998. Morphological polarizations of sensory hair cells in the three otolith organs of a teleost fish: fluorescent labeling of ciliary bundles. Hear. Res. 126: 47-57.

Lu, Z., Song, J., Popper, A.N. 1998. Encoding of acoustic directional information by saccular afferents of the sleeper goby, Dormitator latifrons. J. Comp. Physiol. A 182: 805-815.

Lu, Z., and Popper, A. N. 2001. Neural response directionality correlates of hair cell orientation in a teleost fish. J. Comp. Physiol. A, 187:453-465

Lu, Z., Xu, Z., Buchser, W.J. 2003. Acoustic response properties of lagenar nerve fibers in the sleeper goby, Dormitator latifrons. J. Comp. Physiol. A, 189:889905.

Lu, Z., Xu, Z., Buchser, W.J. 2004. Coding of acoustic particle motion by utricular fibers in the sleeper goby, Dormitator latifrons. J. Comp. Physiol. A, 190:923938.

Lychakov, D.V. 1995. Investigation of the otolithic apparatus in the Acipenser fry. J. Evol. Biochem. Phys. 31: 333-341.

Ma, W.L.D., Fay, R.R. 2002. Neural representations of the axis of acoustic particle motion in nucleus centralis of the torus semicircularis of the goldfish, Carassius auratus. J. Comp. Physiol. (A) 188: 301-313.

Mann, D.A., Lu, Z., Popper, A.N. 1997. Ultrasound detection by a teleost fish. Nature 389:341.

Mann, D.A., Higgs, D.M., Tavolga, WN., Souza, M.J., Popper, A.N. 2001.

Ultrasound detection by clupeiform fishes. J. Acoust. Soc. Am. 109:3048-3054.

Manley, G. 1986. The evolution of the mechanisms of frequency selectivity in vertebrates. In: Moore, B.C., Patterson, R.D. (Eds.). Auditory frequency selectivity. New York, London: Plenum Press, pp. 63-72.

Manley, G. 1990. Peripheral hearing mechanisms of reptiles and birds. Springer Verl. New York.

Manley, G. 2000. The hearing organ of lizards. Dooling, R.J., Popper, A.N., Fay, R.R. (eds.) Comparative hearing: birds and reptiles. Springer-Verlag, New York, pp. 139-196. 
Manley, G., Clack, 2004. An outline of the evolution of vertebrate hearing organs. In: Manley, G., Popper, A.N., Fay, R.R. (Eds.) Evolution of the vertebrate auditory system. Springer-Verlag, New York, pp. 1-26.

Manley, G., Ladher, R. Phylogeny and Evolution of ciliated mechanoreceptor cells. In: Basbaum A.I., Kaneko A., Shepherd G. M., Westheimer G. (Eds.), The Senses: A Comprehensive Reference. Vol. 3, Auditon, Dallos, P., Oertel D. San Diego: Academic Press. pp. 861-870.

Mathiesen, C., Popper, A.N. 1987. The ultrastructure and innervation of the ear of the gar, Lepisosteus osseus. J. Morphol., 194:129-142.

McKibben, J.R., Bass, A.H. 1999. Peripheral encoding of behaviorally relevant acoustic signals in a vocal fish: single tones. J.Comp.Physiol., 184: 563-576.

Meyer M., Popper, A.N. 2002. Hearing in "primitive" fish: brainstem responses to pure tone stimuli in the lake sturgeon, Acipenser fulvescens. Abst. Assn. Res. Otolaryngol., Vol. 25, pp. 11-12.

Meyer M., Plachta, D.T.T., Popper A.N. 2003. When a "primitive" fish listens to tones: encoding of sound in the auditory periphery of the shortnose sturgeon, Acipenser brevirostrum. Abst. Assn. Res. Otolaryngol., Vol. 26, pp. 47-48.

Meyer, M., Popper, A.N., Fay, R.R. 2004. Frequency tuning and directional preferences in lagenar nerve fibers of the goldfish, Carassius auratus. Abst. Assn. Res. Otolaryngol., Vol. 27, p. 325.

Michelsen, A., Larsen, O.N. 2007. Pressure difference receiving ears. Bioinsp. Biomim. 3.

Moeng, R., Popper, A.N. 1984. Auditory responses of saccular neurons of the catfish, Ictalurus punctatus. J. Comp. Physiol., 155:615-624.

Myrberg Jr., A.A., Banner, A., Richard J.D. 1969. Shark attraction using a videoacoustic system. Mar. Biol. 2: 264-276.

Myrberg Jr. A.A., Ha, S.J., Walewski, S., Banbury, J.C. (1972). Effectiveness of acoustic signals in attracting epipelagic sharks to an underwater sound source. Bull. Mar.Sci 22:926-949.

Nelson, J.S. 2006. Fishes of the World. John Wiley: New York.

Nelson, J.S., Johnson, R.H. 1976. Some recent observations on acoustic attraction of Pacific reef sharks. In: Schuijf, A., Hawkins, A.D. (eds). Sound reception in fishes. Amsterdam: Elsevier, pp. 229-239. 
Northcutt, R.G. 1985. The brain and sense organs of the earliest vertebrates: reconstruction of a morphotype. In: Foreman,R.E., Gorbman, A., Dodd, J.m., Olsson, R. (Eds.), Evolutionary Biology of Primitive Fishes. Plenum Press, New York, pp. 81-112.

Northcutt, R.G. 1986. Strategies of comparison: how do we study brain evolution? Verh.dt.zool.Ges., 79:91-103.

Platt, C., Popper, A.N., Fay, R.R. 1989. The ear as part of the octavolateralis system. In: Coombs, S., Görner, P., Münz, M. (Eds.) The mechanosensory lateral line: neurobiology and evolution. Berlin: Springer-Verlag, pp. 633-651.

Platt, C., Jorgensen, J.M., Popper, A.N. 2004. The inner ear of the lungfish, Protopterus. J. Comp. Neurol. 471: 277-278.

Popper, A.N. 1978. Scanning electron microscopic study of the otolithic organs in the bichir (Polypterus bichir) and shovel-nose sturgeon (Scaphirhynchus platorynchus). J. Comp. Neurol., 18:117-128.

Popper, A.N., Salmon, A., Parvulescu A. 1973. Sound localization by the Hawaiian squirrelfishes, Myripristis berndti and M.argyromus. Anim. Behav. 21: 86-97.

Popper, A.N., Coombs, S. 1982. The morphology and evolution of the ear in Actinopterygian fishes. Am. Zool. 22: 311-328.

Popper, A.N., Northcutt, R.G. 1983. Structure and innervation of the inner ear of the bowfin, Amia calva. J. Comp. Neurol., 213:279-286.

Popper, A.N., Platt, C. 1993. Inner ear and lateral line of bony fishes. In: Evans, D.H., (Ed.), The Physiology of Fishes. CRC Press, Boca Raton, FL, pp.99-136.

Popper, A.N., Fay, R.R. 1997. Evolution of the ear and hearing: Issues and questions. Brain Behav.Evol. 50.213-221.

Popper, A.N., Fay, R.R. 1999. The auditory periphery in fishes. In: Fay, R. R. and Popper, A. N. (Eds.) Comparative Hearing: Fish and Amphibians, SpringerVerlag, New York, pp. 43-100.

Popper, A.N., Rogers, P.H., Saidel, W.M., Cox, M. 1988. The role of the fish ear in sound processing. In: Atema, J., Fay, R.R., Popper, A.N., Tavolga, W.N., (Eds.), Sensory Biology of Aquatic Animals, Springer-Verlag, New York, pp. 687-710.

Popper, A.N., Fay, R.R., Platt, C., Sand, O. 2003. Sound detection mechanisms and capabilities of teleost fishes. In: Collin, S.P., Marshall, J.P. (eds). Sensory processing of the aquatic environment. New York: Springer Verlag, pp. 3-23. 
Presson, J.C., Edds, P.E., Popper, A.N. 1992. Central-peripheral and rostral-caudal organization of the innervation of the saccule in a cichlid fish. Brain. Behav. Evol., 39:196-207.

Rafinesque, C.S. 1817. Addition to the observations on the sturgeons of North America. Amer. Mo. Mag., 1:288.

Ramcharitar, J.U., Higgs, D.M., Popper, A.N. 2006. Audition in scianid fishes with different swim bladder-inner ear configurations. J. Acoust. Soc. Am. 439-443.

Retzius, G. 1881. Das Gehörorgan der Wirbelthiere. Vol. I. Samson and Wallin, Stockholm.

Robert, D. 2005. Directional hearing in insects. In: Popper, A.N., Fay, R.R. (Eds.). Sound source localization. Springer-Verlag, New York, pp. 6-35.

Rogers, P., Cox, M. 1988. Underwater sound as a biological stimulus. In: Atema, J., Fay, R.R., Popper, A.N., Tavolga, W.N., (Eds.), Sensory Biology of Aquatic Animals, Springer-Verlag, New York, pp. 131-149.

Ronken, D.A. 1991. Spike discharge properties that are related to the characteristic frequency of single units in the frog auditory nerve. J. Acoust. Soc. Am. 90: 2428-2440.

Ruggero, M., 1992. Physiology and coding of sound in the auditory nerve. In: Popper, A.N., Fay, R.R. (Eds.). The mammalian auditory pathway: Neurophysiology. Springer-Verlag, New York, pp. 34-93.

Saffron, I. 2002. Caviar, the strange history and uncertain future of the world's most coveted delicacy. Broadway books, New York.

Sand, O. 1974. Directional sensitivity of microphonic potentials from the perch. J.Exp.Biol. 60:881-899.

Sand, O., Enger, P.S. 1973. Evidence for an auditory function of the swim bladder in the cod. J. Exp.Biol.59: 405-414.

Sand, O., Karlsen, H.E. 2000. Detection of infrasound and linear acceleration in fish. Phil.Trans.R.Soc.Lond.B 355:1295-1298.

Sand, O., Bleckmann, H. 2008. Orientation to auditory and lateral line stimuli. In: Webb, J.F., Fay, R.R., Popper, A.N. (Eds.), Fish Bioacoustics, Springer-Verlag, New York, pp. 183-232. 
Scheifele, P.M., Darre, M. 2005. Noise levels and sources in the Stellwagen Bank National Marine Sanctuary and the St. Lawrence River Estuary. National Marine Sanctuary and the St. Lawrence River Estuary. Marine Conservation Series MSD-05-1. U. S. Department of Commerce, National Oceanic and Atmospheric Administration, Marine Sanctuaries Devision, Silver Spring. 26 pp.

Schuijf, A., Baretta, J.W., Wildschutt, J.T. 1972. A field investigation on the discrimination of sound direction in Labrus berggylta (Pisces: Perciformes). Netherlands J. Zool. 22: 81-104.

Schuijf, A. 1975. Directional hearing of cod (Gadus morhua) under approximate free field conditions. J. Comp. Physiol. 98, 307-332.

Schuijf, A., Buwalda, R.J.A. 1975. On the mechanism of directional hearing in cod (Gadus morhua). J.Comp.Physiol. A. 98: 333-344.

Sento, S., Furukawa, T. 1987. Intra-axonal labeling of saccular afferents in the goldfish, Carassius auratus: correlations between morphological and physiological characteristics. J. Comp. Neurol. 258: 352-367.

Sillman, A.J., Ong, E.K., Loew, E.R. 2007. Spectral absorbance, structure, and population of photoreceptors in the lake sturgeon, Acipenser fulvescens. Can. J. Zool. 85: 584-587.

Simmons, D.D., Meenderink, W.F., Vassilakis, P.N. 2007. Anatomy, physiology, and function of auditory end organs in the frog inner ear. In: Narins, P.M., Feng, A.S., Popper, A.N., Fay, R.R. (Eds.). Hearing and sound communication in amphibians. Springer-Verlag, New York, pp. 184-220.

Spoendlin, H. 1964. Organization of the sensory hairs in the gravity receptors in the utricle and saccule of the squirrel monkey. Z. Zellforsch. 62:701-716.

Sugihara, I. \& Furukawa, T., 1989. Morphological and functional aspects of two different types of hair cells in the goldfish sacculus. J. Neurophysiol. 62:13301343.

Suzuki, A., Kozloski, J., Crawford J.D. 2002. Temporal encoding for auditory computation: physiology of primary afferent neurons in sound-producing fish. J.Neurosc. 22:6290-6301.

Tautz, J. 1977. Reception of medium vibration by thoracal hairs of the caterpillar of Barathra brassicae L. (Lepidoptera, Noctuidae). I. Mechanical properties of the receptor hairs. J.Comp.Physiol. 118:13-31. 
van Bergeijk, W.A. 1964. Directional and non-directional hearing in fish. In:

Tavolga, W.A. (ed). Marine Bioacoustics. Pergamon Press, London, pp. 269301.

van Bergeijk, W.A. 1967. The evolution of vertebrate hearing. In: Neff, W.D. (ed.). Contributions to Sensory Physiology. Academic Press, New York pp.1-49.

von Békésy, G.1960. Experiments in hearing. New York: McGraw-Hill.

von Frisch, K., Dijkgraaf, S. 1935. Can fish perceive sound direction? Z. vergl. Physiol. 22: 641-655.

Weeg, M.S., Fay, R.R., Bass, A.H. 2002. Directional response and frequency tuning in saccular nerve fibers of a vocal fish, Porychthus notatus. J.Comp.Physiol. A. 188: 631-641.

Wever, E.G. 1949. Theory of Hearing. New York: Wiley.

Wiley, E.O. 1981. Phyologenetics - The theory and practice of phylogenetic systematics. John Wiley and Sons, New York. 